\title{
Maximal almost disjoint families of functions
}

\author{
by
}

Dilip Raghavan (Toronto)

\begin{abstract}
We study maximal almost disjoint (MAD) families of functions in $\omega^{\omega}$ that satisfy certain strong combinatorial properties. In particular, we study the notions of strongly and very MAD families of functions. We introduce and study a hierarchy of combinatorial properties lying between strong MADness and very MADness. Proving a conjecture of Brendle, we show that if $\operatorname{cov}(\mathcal{M})<\mathfrak{a}_{\mathfrak{e}}$, then there no very MAD families. We answer a question of Kastermans by constructing a strongly MAD family from $\mathfrak{b}=\mathfrak{c}$. Next, we study the indestructibility properties of strongly MAD families, and prove that the strong MADness of strongly MAD families is preserved by a large class of posets that do not make the ground model reals meager. We solve a well-known problem of Kellner and Shelah by showing that a countable support iteration of proper posets of limit length does not make the ground model reals meager if no initial segment does. Finally, we prove that the weak Freese-Nation property of $\mathcal{P}(\omega)$ implies that all strongly MAD families have size at most $\aleph_{1}$.
\end{abstract}

1. Introduction. In this paper, we study the notion of a strongly MAD family of functions in $\omega^{\omega}$, as well as several variations of this notion, such as very MAD families. We solve a variety of problems about these notions. Recall that two functions $f$ and $g$ in $\omega^{\omega}$ are said to be almost disjoint or a.d. if $|f \cap g|<\omega$. A family $\mathscr{A} \subset \omega^{\omega}$ is said to a.d. if its members are pairwise a.d. And finally, an a.d. family $\mathscr{A} \subset \omega^{\omega}$ is said to be maximal almost disjoint or $M A D$ if $\forall f \in \omega^{\omega} \exists h \in \mathscr{A}[|h \cap f|=\omega]$.

The notion of a strongly MAD family is a " $\sigma$ version" of the notion of a MAD family of functions. Very roughly, this means that instead of requiring the family to be maximal just with respect to elements of $\omega^{\omega}$, we require it to be maximal with respect to countable subsets of $\omega^{\omega}$. " $\sigma$ versions" of various types of subfamilies of $[\omega]^{\omega}=\{a \subset \omega:|a|=\omega\}$ have been considered in the literature. For example, Malykhin [19] and Kam-

2000 Mathematics Subject Classification: 03E17, 03E15, 03E35, 03E40, 03E05, 03E50, $03 \mathrm{E} 65$.

Key words and phrases: maximal almost disjoint family, cardinal invariants, iterated forcing, preservation theorem, weak Freese-Nation property. 
burelis and Weglorz [13] have studied the " $\sigma$ version" of the notion of a splitting family. Recall that a set $a \in[\omega]^{\omega}$ splits a set $b \in[\omega]^{\omega}$ if both $a \cap b$ and $b \backslash a$ are infinite. Recall also that a family $\mathcal{F} \subset[\omega]^{\omega}$ is a splitting family if every $b \in[\omega]^{\omega}$ is split by some $a \in \mathcal{F}$. Now, the " $\sigma$ version" of this notion, called an $\aleph_{0}$-splitting family, is simply a family $\mathcal{F} \subset[\omega]^{\omega}$ such that for every countable set $\left\{b_{i}: i \in \omega\right\} \subset[\omega]^{\omega}$, there is a single $a \in \mathcal{F}$ which splits all the $b_{i}$. We cannot simply lift this definition to the case of MAD families. That is, we cannot define a strongly MAD family to simply be an a.d. family $\mathscr{A} \subset \omega^{\omega}$ such that for every countable set of functions $\left\{f_{i}: i \in \omega\right\} \subset \omega^{\omega}$, there is $h \in \mathscr{A}$ such that $\forall i \in \omega\left[\left|h \cap f_{i}\right|=\omega\right]$. To see this, suppose $\mathscr{A} \subset \omega^{\omega}$ is an a.d. family with at least two elements. Choose $h_{0}, h_{1} \in \mathscr{A}$, with $h_{0} \neq h_{1}$, and consider the set $\left\{h_{0}, h_{1}\right\}$. It is clear that no element of $\mathscr{A}$ can intersect both $h_{0}$ and $h_{1}$ in an infinite set. Hence we must put some restriction on the countable sets of functions we are allowed to consider. It is easy to see that the above counterexample will go through even if $h_{0}$ and $h_{1}$ are covered by finitely many members from $\mathscr{A}$.

Definition 1. Let $\mathscr{A} \subset \omega^{\omega}$ be an a.d. family, and let $Y \in[\omega \times \omega]^{\omega}$. We say that $Y$ avoids $\mathscr{A}$ if for every finite set $\left\{h_{0}, \ldots, h_{k}\right\} \subset \mathscr{A}, \mid Y \backslash\left(h_{0} \cup \ldots\right.$ $\left.\cup h_{k}\right) \mid=\omega$.

Definition 2. Let $\mathscr{A} \subset \omega^{\omega}$ be an a.d. family. We say that $\mathscr{A}$ is strongly $M A D$ if for any countable family of functions $\left\{f_{i}: i \in \omega\right\} \subset \omega^{\omega}$ avoiding $\mathscr{A}$, there is $h \in \mathscr{A}$ such that $\forall i \in \omega\left[\left|h \cap f_{i}\right|=\omega\right]$.

Strongly MAD families were introduced by Steprāns [15], who showed that they cannot be analytic, though the same notion had been considered earlier by Malykhin [19] in the context of MAD families of subsets of $\omega$ and further studied by Kurilić [18] and Hrušák and García Ferreira [9]. Soon after, Zhang and Kastermans [14] introduced a strengthening of this notion, which they called very MAD family.

Definition 3. Let $\mathscr{A} \subset \omega^{\omega}$ be an a.d. family, and let $\kappa=|\mathscr{A}|$. We say that $\mathscr{A}$ is very $M A D$ if for all cardinals $\lambda<\kappa$ and for every family $\left\{f_{\alpha}: \alpha<\lambda\right\} \subset \omega^{\omega}$ of functions avoiding $\mathscr{A}$, there is $h \in \mathscr{A}$ such that $\forall \alpha<\lambda\left[\left|f_{\alpha} \cap h\right|=\omega\right]$.

Clearly, very MAD families are strongly MAD, which in turn, are MAD. On the other hand, E. van Douwen [20] asked about the existence of the following sort of MAD family in $\omega^{\omega}$ :

DeFinition 4. Let us say that $p$ is an infinite partial function if there is an infinite set $X \in[\omega]^{\omega}$ such that $p \in \omega^{X}$. An a.d. family $\mathscr{A} \subset \omega^{\omega}$ is said to be van Douwen $M A D$ if for every infinite partial function $p$, there is $h \in \mathscr{A}$ such that $|p \cap h|=\omega$. 
It turns out that the notion of a van Douwen MAD family is weaker than that of a strongly MAD family (see Lemma 6). Thus, we have a natural spectrum of combinatorial properties of increasing strength. In this paper we investigate this hierarchy and answer several questions about these notions. In [21] we positively answered van Douwen's question by showing in ZFC that van Douwen MAD families exist.

In Section 2 we first refine this hierarchy by introducing a sequence of combinatorial properties lying between MADness and very MADness, and we show under $\mathrm{MA}(\sigma$-centered $)$ that this refined hierarchy is proper, meaning that there is a member in each class not belonging to any higher class.

Sections 3 and 4 answer questions of Kastermans and Brendle regarding when very MAD families exist and whether it is possible to have models with strongly MAD families, but no very MAD families. In Section 3, we modify a construction of Hrušák [8], Kurilić [18] and Brendle and Yatabe [4] to show that strongly MAD families exist if $\mathfrak{b}=\mathfrak{c}$. In Section 4 , we prove a conjecture of Brendle that if $\operatorname{cov}(\mathcal{M})<\mathfrak{a}_{\mathfrak{e}}$, there are no very MAD families. Together these results show that in the Laver model there is a strongly MAD family, but no very MAD families.

In Sections 5 and 6 we study the effect of forcing on strongly MAD families in $\omega^{\omega}$. In particular, in Section 5, we are interested in characterizing those posets that preserve the strong MADness of strongly MAD families. While we do not provide a full characterization, we show that many posets that do not turn the set of ground model reals into a meager set also preserve the strong MADness of strongly MAD families (it is easy to see that not making the ground model reals meager is a necessary condition for a poset to do this). We introduce a property of posets called having diagonal fusion (see Definition 40), and we show that all posets that have diagonal fusion preserve the strong MADness of all strongly MAD families. The class of posets having diagonal fusion includes all the usual posets not making the ground model reals meager, like Sacks and Miller forcing. Given this close connection between these two properties, we conjecture (see Conjecture 30) that they actually coincide for a class of "nicely definable" posets.

In Section 6 we solve a problem from Kellner and Shelah [16] regarding the preservation of the property of not making the ground model reals meager under countable support iterations. Preservation theorems are an important component of the theory of iterated forcing. The simplest and most well-known preservation theorem says that the finite support iteration of c.c.c. posets is c.c.c. For countable support iterations (CS iterations), a fundamental result of Shelah [23] is that properness is preserved under such iterations. Other well-known examples for CS iterations include the preservation of $\omega^{\omega}$-bounding and of the Sacks property. For a given property $\mathbf{P}$ of 
forcing notions two kinds of preservation may be considered. One kind says if $\left\langle\mathbb{P}_{\alpha}, \mathbb{Q}_{\alpha}: \alpha \leq \gamma\right\rangle$ is a CS iteration such that for each $\alpha<\gamma, \Vdash_{\alpha} " \mathbb{Q}_{\alpha}$ is proper and has property $\mathbf{P}$ ", then $\mathbb{P}_{\gamma}$ also has property $\mathbf{P}$. An example of this kind is the preservation of $\omega^{\omega}$-bounding. The other kind of preservation theorem say that given a limit ordinal $\gamma$, if $\left\langle\mathbb{P}_{\alpha}, \mathbb{Q}_{\alpha}: \alpha \leq \gamma\right\rangle$ is a CS iteration such that for each $\alpha<\gamma, \Vdash_{\alpha}$ " $\mathbb{Q}_{\alpha}$ is proper" and $\mathbb{P}_{\alpha}$ has property $\mathbf{P}$, then $\mathbb{P}_{\gamma}$ also has property $\mathbf{P}$. An example of this kind is the preservation of not adding random reals. The key difference is that in the first kind of preservation, $\mathbf{P}$ is assumed to hold for each iterand, whereas in the second kind, $\mathbf{P}$ is assumed to hold for each initial segment of the iteration (in both cases the iterands are assumed to be proper). One often considers a preservation theorem of the second kind only for properties $\mathbf{P}$ that fail to be preserved by two-step iterations, so that one cannot hope for a result of the first kind for them. In [16] Kellner and Shelah asked if the second kind of preservation theorem holds for the properties of not making $\mathbf{V} \cap \omega^{\omega}$ meager and not making $\mathbf{V} \cap \omega^{\omega}$ null. Regarding the first of these, Kellner and Shelah [16] prove that it holds under the additional assumption that the iterands are nep, providing a partial positive answer. Also, it is shown in Goldstern [7] that the first kind of preservation theorem holds for the property of preserving $\sqsubseteq_{\mathbf{C}}$ (see Definition 27), which is a property stronger than not making $\mathbf{V} \cap \omega^{\omega}$ meager. In Section 6 we answer the question of Kellner and Shelah affirmatively by proving the most general version of the second kind of preservation theorem for the property of not making $\mathbf{V} \cap \omega^{\omega}$ meager. We then use this result to show that any countable support iteration of posets having diagonal fusion preserves the strong MADness of all strongly MAD families.

In Section 7 we show that the weak Freese-Nation property of $\mathcal{P}(\omega)$ implies that all strongly MAD families have size at most $\aleph_{1}$. The weak FreeseNation property of $\mathcal{P}(\omega)$ is one of several axioms considered by Juhász and others (see [12]) in an attempt to capture the combinatorial essence of the Cohen model. This axiom is known to be true in this and several other models. Thus, in particular, there are no strongly MAD families of size $\mathfrak{c}$ in the Cohen model.

Finally, in Section 8 we end with some miscellaneous observations concerning some similarities and differences between the notion of a strongly MAD family of functions in $\omega^{\omega}$ and that of sets in $[\omega]^{\omega}$.

2. The strength of an a.d. family. We will introduce the notion of strength of an a.d. family of functions. This notion allows for the systematic investigation of variations on the concept of a strongly MAD family. In particular, it introduces a sequence of combinatorial properties of intermediate strength between MADness and very MADness, with strong MADness as a special case. The notion of strength allows for the systematic investigation 
of this entire spectrum starting with MADness, going through van Douwen and strong MADness, all the way up to very MADness.

Definition 5. Let $\mathscr{A} \subset \omega^{\omega}$ be an a.d. family. We define the strength of $\mathscr{A}$, written $\operatorname{st}(\mathscr{A})$, to be the least cardinal $\kappa$ such that there is a family $\left\{f_{\alpha}: \alpha<\kappa\right\} \subset \omega^{\omega}$ of functions avoiding $\mathscr{A}$ such that $\forall h \in \mathscr{A} \exists \alpha<\kappa$ $\left[\left|h \cap f_{\alpha}\right|<\omega\right]$.

Thus an a.d. family $\mathscr{A}$ is MAD iff $\operatorname{st}(\mathscr{A}) \geq 2$. It is strongly MAD iff $\operatorname{st}(\mathscr{A}) \geq \omega_{1}$, and it is very $\mathrm{MAD}$ iff $\operatorname{st}(\mathscr{A}) \geq|\mathscr{A}|$. The next lemma points out a connection with the notion of a van Douwen MAD family.

Lemma 6. Let $\mathscr{A} \subset \omega^{\omega}$ be an a.d. family. If $\operatorname{st}(\mathscr{A}) \geq 3$, then $\mathscr{A}$ is van Douwen MAD.

Proof. Suppose, for a contradiction, that there is an infinite partial function $f$ from $\omega$ to $\omega$ which is a.d. from $\mathscr{A}$. Let $h_{0} \neq h_{1}$ be two distinct functions in $\mathscr{A}$. Let $a=\operatorname{dom}(f)$ and let $b=\omega \backslash a$. Let $g_{0}=f \cup h_{0} \uparrow b$ and let $g_{1}=f \cup h_{1} \uparrow b$. Since $f$ is a.d. from $\mathscr{A}$, it avoids $\mathscr{A}$. So $\left\{g_{0}, g_{1}\right\} \subset \omega^{\omega}$ is a set of two functions avoiding $\mathscr{A}$. As st $(\mathscr{A}) \geq 3$, there is $h \in \mathscr{A}$ such that $\left|h \cap g_{0}\right|=\left|h \cap g_{1}\right|=\omega$. We will argue that $|f \cap h|=\omega$, giving a contradiction. Indeed, suppose that $|f \cap h|<\omega$. Since $h$ intersects both $g_{0}$ and $g_{1}$ in an infinite set, it follows that both $h \cap h_{0}$ and $h \cap h_{1}$ are infinite. But since $\mathscr{A}$ is an a.d. family, this implies that $h=h_{0}$ and $h=h_{1}$, contradicting our choice of $h_{0}$ and $h_{1}$.

This argument can be generalized to yield the following.

Lemma 7. Let $\kappa$ be an infinite cardinal. Let $\mathscr{A} \subset \omega^{\omega}$ be an a.d. family with $\operatorname{st}(\mathscr{A})>\kappa$. If $\left\{f_{\alpha}: \alpha<\kappa\right\}$ is a family of infinite partial functions avoiding $\mathscr{A}$, there is $h \in \mathscr{A}$ such that $\forall \alpha<\kappa\left[\left|h \cap f_{\alpha}\right|=\omega\right]$.

Proof. Just as in Lemma 6, pick two distinct members $h_{0}$ and $h_{1}$ of $\mathscr{A}$. For each $\alpha<\kappa$, set $a_{\alpha}=\operatorname{dom}\left(f_{\alpha}\right)$ and $b_{\alpha}=\omega \backslash a_{\alpha}$. Put $g_{\alpha}^{0}=\left(f_{\alpha} \cup h_{0}\right)\left\lceil b_{\alpha}\right.$ and $g_{\alpha}^{1}=\left(f_{\alpha} \cup h_{1}\right)\left\lceil b_{\alpha}\right.$. Since $f_{\alpha}$ avoids $\mathscr{A}$, both $g_{\alpha}^{0}$ and $g_{\alpha}^{1}$ avoid $\mathscr{A}$. As $\kappa$ is an infinite cardinal, $\left\{g_{\alpha}^{i}: i \in 2 \wedge \alpha<\kappa\right\} \subset \omega^{\omega}$ is a family of $\kappa$ functions avoiding $\mathscr{A}$. As $\operatorname{st}(\mathscr{A})>k$, there is $h \in \mathscr{A}$ such that $\forall \alpha<\kappa \forall i \in 2$ $\left[\left|h \cap g_{\alpha}^{i}\right|=\omega\right]$. Now, it is easily argued, just as in Lemma 6, that $\forall \alpha<\kappa$ $\left[\left|h \cap f_{\alpha}\right|=\omega\right]$.

It is natural to ask for which sets of cardinals $X \subset \mathfrak{c}+1$ it is consistent to have $X=\left\{\operatorname{st}(\mathscr{A}): \mathscr{A} \subset \omega^{\omega}\right.$ is an a.d. family $\}$. We will provide a partial answer by showing that under $\mathrm{MA}(\sigma$-centered $)$, every cardinal $\kappa \leq \mathfrak{c}$ occurs as the strength of some a.d. family $\mathscr{A}$. We first point out that the strength of an infinite a.d. family can never exceed its size. In Section 4 we obtain a better upper bound by showing that $\operatorname{st}(\mathscr{A}) \leq \operatorname{cov}(\mathcal{M})$ (Corollary 25)

Lemma 8. Let $\mathscr{A} \subset \omega^{\omega}$ be an infinite a.d. family. Then $\operatorname{st}(\mathscr{A}) \leq|\mathscr{A}|$. 
Proof. For each $h \in \mathscr{A}$ choose a countably infinite set $X_{h} \in[\mathscr{A}]^{\omega}$ with $h \notin X_{h}$. Pick $g_{h} \in \omega^{\omega}$ such that $g_{h}$ is a.d. from $h$ but $\forall h^{\prime} \in X_{h}\left[\left|h^{\prime} \cap g_{h}\right|=\omega\right]$. It is possible to do this because $h$ is a.d. from everything in $X_{h}$. Now since $g_{h}$ has infinite intersection with infinitely many members of $\mathscr{A}$, it avoids $\mathscr{A}$. Thus $\left\{g_{h}: h \in \mathscr{A}\right\}$ is a family of functions avoiding $\mathscr{A}$ of size at most $|\mathscr{A}|$ such that no member of $\mathscr{A}$ has infinite intersection with all the functions in the family.

In what follows, we prove that when $\kappa \leq \mathfrak{c}$, there is an a.d. family $\mathscr{A} \subset \omega^{\omega}$ with $\operatorname{st}(\mathscr{A})=\kappa$ (Theorem 11). To get $\operatorname{st}(\mathscr{A}) \leq \kappa$, we fix an a.d. family $\left\langle f_{\alpha}: \alpha<\kappa\right\rangle \subset \omega^{\omega}$ ahead of time, and we ensure during the construction that this family avoids $\mathscr{A}$, and yet that no member of $\mathscr{A}$ has infinite intersection with all the $f_{\alpha}$. To get $\operatorname{st}(\mathscr{A}) \geq \kappa$, we recursively take care of all subsets of $\omega^{\omega}$ of size less than $\kappa$. At any stage, given a set $\left\langle g_{\alpha}: \alpha<\lambda\right\rangle$ of functions of size less than $\kappa$ avoiding $\mathscr{A}$, we will use $\mathrm{MA}(\sigma$-centered) to find a function a.d. from $\mathscr{A}$ and having infinite intersection with all the $g_{\alpha}$. The following lemma is crucial for ensuring that we can do this without violating the first requirement.

Lemma 9. Let $\lambda<\kappa$ be cardinals. Let $\mathscr{A} \subset \omega^{\omega}$ be a family of functions and let $\left\langle f_{\alpha}: \alpha<\kappa\right\rangle \subset \omega^{\omega}$ be an a.d. family. Let $\left\langle g_{\alpha}: \alpha<\lambda\right\rangle \subset \omega^{\omega}$ be any family of functions avoiding $\mathscr{A}$. There is an $\alpha<\kappa$ such that $\left\langle g_{\alpha}: \alpha<\lambda\right\rangle$ avoids $\mathscr{A} \cup\left\{f_{\alpha}\right\}$.

Proof. Suppose not. Then for each $\alpha<\kappa$ there is a $\beta<\lambda$ and a finite subset $\left\{h_{0}, \ldots, h_{k}\right\} \subset \mathscr{A}$ such that $g_{\beta} \subset^{*} h_{0} \cup \cdots \cup h_{k} \cup f_{\alpha}$. As $\lambda<\kappa$, it follows that there are distinct $\alpha_{0} \neq \alpha_{1}<\kappa$ such that for the same $\beta<\lambda$ there are finite sets $\left\{h_{0}, \ldots, h_{k}\right\} \subset \mathscr{A}$ and $\left\{h^{0}, \ldots, h^{l}\right\} \subset \mathscr{A}$ so that both $g_{\beta} \subset^{*} h_{0} \cup \cdots \cup h_{k} \cup f_{\alpha_{0}}$ and $g_{\beta} \subset^{*} h^{0} \cup \cdots \cup h^{l} \cup f_{\alpha_{1}}$ hold. By assumption, $g_{\beta}$ avoids $\mathscr{A}$. Therefore, $p=g_{\beta} \backslash\left(h_{0} \cup \cdots \cup h_{k} \cup h^{0} \cup \cdots \cup h^{l}\right)$ is an infinite partial function. But now, it follows that $p \subset^{*} f_{\alpha_{0}}$ and $p \subset^{*} f_{\alpha_{1}}$, which is a contradiction because $\left\langle f_{\alpha}: \alpha<\kappa\right\rangle$ is an a.d. family.

Lemma 10. Assume $M A(\sigma$-centered $)$. Let $\mathscr{A} \subset \omega^{\omega}$ be an a.d. family with $|\mathscr{A}|<\mathfrak{c}$. Let $\lambda<\kappa<\mathfrak{c}$ be cardinals. Let $\left\langle f_{\alpha}: \alpha<\kappa\right\rangle \subset \omega^{\omega}$ be an a.d. family of functions avoiding $\mathscr{A}$. Let $\left\langle g_{\alpha}: \alpha<\lambda\right\rangle \subset \omega^{\omega}$ be another family of functions also avoiding $\mathscr{A}$. There is $h \in \omega^{\omega}$ such that:

(1) $\forall h^{\prime} \in \mathscr{A}\left[\left|h \cap h^{\prime}\right|<\omega\right]$.

(2) $\left\langle f_{\alpha}: \alpha<\kappa\right\rangle$ avoids $\mathscr{A} \cup\{h\}$.

(3) $\forall \beta<\lambda\left[\left|h \cap g_{\beta}\right|=\omega\right]$.

(4) $\exists \alpha<\kappa\left[\left|h \cap f_{\alpha}\right|<\omega\right]$.

Proof. By Lemma 9, there is $\alpha<\kappa$ such that $\left\langle g_{\beta}: \beta<\lambda\right\rangle$ avoids $\mathscr{B}=$ $\mathscr{A} \cup\left\{f_{\alpha}\right\}$. Let us fix such an $\alpha$. Let $\operatorname{Fn}(\omega, \omega)$ denote the set of finite partial functions from $\omega$ to $\omega$. Consider the poset $\mathbb{P}=\{\langle s, H\rangle: s \in \operatorname{Fn}(\omega, \omega) \wedge H$ 
$\left.\in[\mathscr{B}]^{<\omega}\right\}$. We order $\mathbb{P}$ as follows: given $\left\langle s_{0}, H_{0}\right\rangle$ and $\left\langle s_{1}, H_{1}\right\rangle$ in $\mathbb{P},\left\langle s_{0}, H_{0}\right\rangle \leq$ $\left\langle s_{1}, H_{1}\right\rangle$ iff $s_{0} \supset s_{1} \wedge H_{0} \supset H_{1} \wedge \forall h \in H_{1} \forall n \in \operatorname{dom}\left(s_{0}\right) \backslash \operatorname{dom}\left(s_{1}\right)[h(n) \neq$ $s_{0}(n)$ ]. It is easily checked that $\mathbb{P}$ is $\sigma$-centered. If $G \subset \mathbb{P}$ is a filter on $\mathbb{P}$, then $h=\bigcup\{s: \exists H[\langle s, H\rangle \in G]\}$ is a function, which is a.d. from $\bigcup\{H$ : $\exists s[\langle s, H\rangle \in G]\}$. To see that we can get a function $h$ that satisfies the necessary requirements, we will check that certain sets are dense.

- To ensure $h \in \omega^{\omega}$ : For each $n \in \omega$, set $D_{n}=\{\langle s, H\rangle \in \mathbb{P}: n \in$ $\operatorname{dom}(s)\}$. We will check that $D_{n}$ is dense. Fix $\left\langle s_{0}, H_{0}\right\rangle \in \mathbb{P}$. If $n \in$ $\operatorname{dom}\left(s_{0}\right)$, there is nothing to be done. Otherwise, $\left\{h^{\prime}(n): h^{\prime} \in H_{0}\right\}$ is a finite subset of $\omega$. So we may choose $k \in \omega \backslash\left\{h^{\prime}(n): h^{\prime} \in H_{0}\right\}$. Now, $\left\langle s_{0} \cup\{\langle n, k\rangle\}, H_{0}\right\rangle$ is an extension of $\left\langle s_{0}, H_{0}\right\rangle$ in $D_{n}$.

- To ensure $h$ satisfies requirements (1) and (4): It is enough to ensure that $\forall h^{\prime} \in \mathscr{B} \exists\langle s, H\rangle \in G\left[h^{\prime} \in H\right]$. But it is obvious that for each $h^{\prime} \in \mathscr{B}, D_{h^{\prime}}=\left\{\langle s, H\rangle \in \mathbb{P}: h^{\prime} \in H\right\}$ is dense.

- To ensure $h$ satisfies requirement (2): Let $F$ be a finite subset of $\mathscr{A}$. Let $\gamma<\kappa$. Since $f_{\gamma}$ avoids $\mathscr{A}, X_{\gamma}^{F}=\left\{n \in \omega: \forall h^{\prime} \in F\left[f_{\gamma}(n) \neq h^{\prime}(n)\right]\right\}$ is an infinite subset of $\omega$. For each $n \in \omega$, consider the set $D(F, \gamma, n)=$ $\left\{\langle s, H\rangle \in \mathbb{P}: \exists m>n\left[m \in X_{\gamma}^{F} \wedge m \in \operatorname{dom}(s) \wedge f_{\gamma}(m) \neq s(m)\right]\right\}$. If $G$ hits $D(F, \gamma, n)$ for all $n$, then $f_{\gamma}$ avoids $F \cup\{h\}$ because there will be infinitely many $m \in X_{\gamma}^{F}$ so that $h(m) \neq f_{\gamma}(m)$. To see that $D(F, \gamma, n)$ is dense, fix $\left\langle s_{0}, H_{0}\right\rangle \in \mathbb{P}$. Since $X_{\gamma}^{F}$ is an infinite set, there is $m \in X_{\gamma}^{F}$ which is greater than $n$ and outside $\operatorname{dom}\left(s_{0}\right)$. Now, we can choose $k \notin\left\{h^{\prime}(m): h^{\prime} \in H_{0}\right\} \cup\left\{f_{\gamma}(m)\right\}$. It is clear that $\left\langle s_{0} \cup\{\langle m, k\rangle\}, H_{0}\right\rangle$ is as required.

- To ensure $h$ satisfies requirement (3): Let $\beta<\lambda$. It is enough to make $G$ intersect $D_{n}^{\beta}=\left\{\langle s, H\rangle \in \mathbb{P}: \exists m>n\left[m \in \operatorname{dom}(s) \wedge s(m)=g_{\beta}(m)\right]\right\}$ for all $n \in \omega$. To see that this set is dense, fix $\left\langle s_{0}, H_{0}\right\rangle \in \mathbb{P}$. We know, by our choice of $\alpha$, that $g_{\beta}$ avoids $\mathscr{B}$. So there are infinitely many $m \in \omega$ such that $\forall h^{\prime} \in H_{0}\left[h^{\prime}(m) \neq g_{\beta}(m)\right]$. So we can choose such an $m$ greater than $n$ and outside of $\operatorname{dom}\left(s_{0}\right)$. By our choice of $m$, $\left\langle s_{0} \cup\left\{\left\langle m, g_{\beta}(m)\right\rangle\right\}, H_{0}\right\rangle$ extends $\left\langle s_{0}, H_{0}\right\rangle$ and is as required.

Since $\lambda<\kappa<\mathfrak{c}$ and $|\mathscr{A}|<\mathfrak{c}$ and since MA( $\sigma$-centered $)$ is assumed, we can find a filter $G$ that intersects all the sets in $\left\{D_{n}: n \in \omega\right\} \cup\left\{D_{h^{\prime}}: h^{\prime} \in \mathscr{B}\right\}$ $\cup\left\{D_{n}^{\beta}: \beta<\lambda \wedge n \in \omega\right\} \cup\left\{D(F, \gamma, n): F \in[\mathscr{A}]^{<\omega} \wedge \gamma<\kappa \wedge n \in \omega\right\}$. Now, $h$, defined as above from $G$, will have the required properties.

Theorem 11. Assume $M A(\sigma$-centered $)$. Let $\kappa \leq \mathfrak{c}$ be a cardinal. There is an a.d. family $\mathscr{A} \subset \omega^{\omega}$ with $\operatorname{st}(\mathscr{A})=\kappa$.

Proof. We first deal with the case when $\kappa=\mathfrak{c}$. Kastermans [14] showed that there is a very MAD family under $\operatorname{MA}(\sigma$-centered $)$. Let $\mathscr{A}$ be a very 
MAD family. Clearly, $\mathfrak{c}=|\mathscr{A}| \leq \operatorname{st}(\mathscr{A})$, and by Lemma 8 , st $(\mathscr{A}) \leq|\mathscr{A}|=\mathfrak{c}$, whence $\operatorname{st}(\mathscr{A})=\mathfrak{c}$.

Now let us assume that $\kappa<\mathfrak{c}$. Fix an a.d. family $\left\langle f_{\alpha}: \alpha<\kappa\right\rangle \subset \omega^{\omega}$ of size $\kappa$. We will construct an a.d. family $\mathscr{A} \subset \omega^{\omega}$ with $\operatorname{st}(\mathscr{A}) \geq \kappa$, while at the same time ensuring that $\left\langle f_{\alpha}: \alpha<\kappa\right\rangle$ avoids $\mathscr{A}$, and yet nothing in $\mathscr{A}$ has infinite intersection with all the $f_{\alpha}$. Thus $\left\langle f_{\alpha}: \alpha<\kappa\right\rangle$ will witness that $\operatorname{st}(\mathscr{A})=\kappa$.

$\mathscr{A}$ will be the union of an increasing sequence of a.d. families. Since $\mathrm{MA}(\sigma$-centered $)$ is assumed, $\mathfrak{c}^{<\kappa}=\mathfrak{c}$. So we can let $\left\langle\mathscr{G}_{\alpha}: \alpha<\mathfrak{c}\right\rangle$ enumerate all subsets of $\omega^{\omega}$ of size less than $\kappa$. We will construct a sequence $\left\langle\mathscr{A}_{\alpha}: \alpha<\mathfrak{c}\right\rangle$ so that:

(1) $\mathscr{A}_{\alpha} \subset \omega^{\omega}$ is an a.d. family of size $\leq|\alpha|$.

(2) $\forall \alpha<\beta<\mathfrak{c}\left[\mathscr{A}_{\alpha} \subset \mathscr{A}_{\beta}\right]$.

(3) If $\mathscr{G}_{\alpha}$ avoids $\bigcup\left\{\mathscr{A}_{\beta}: \beta<\alpha\right\}$, then $\exists h \in \mathscr{A}_{\alpha} \forall g \in \mathscr{G}_{\alpha}[|h \cap g|=\omega]$.

(4) $\left\langle f_{\alpha}: \alpha<\kappa\right\rangle$ avoids $\mathscr{A}_{\alpha}$.

(5) $\forall h \in \mathscr{A}_{\alpha} \exists \beta<\kappa\left[\left|h \cap f_{\beta}\right|<\omega\right]$.

Assume that $\left\langle\mathscr{A}_{\beta}: \beta<\alpha\right\rangle$ is already given to us. Let $\mathscr{B}=\bigcup \mathscr{A}_{\beta}$. If $\mathscr{G}_{\alpha}$ does not avoid $\mathscr{B}$, there is nothing to be done. In this case, we simply set $\mathscr{A}_{\alpha}=\mathscr{B}$. Let us assume from now on that $\mathscr{G}_{\alpha}$ avoids $\mathscr{B}$. Notice that by clause (4), $\left\langle f_{\alpha}: \alpha<\kappa\right\rangle$ avoids $\mathscr{B}$ as well. By clause (1), $\mathscr{B}$ is an a.d. family with $|\mathscr{B}|<\mathfrak{c}$. Let $\lambda=\left|\mathscr{G}_{\alpha}\right|$. Note that we have $\lambda<\kappa<\mathfrak{c}$. Now, we can apply Lemma 10 with $\mathscr{B}$ as $\mathscr{A}$ and $\mathscr{G}_{\alpha}$ as $\left\langle g_{\alpha}: \alpha<\lambda\right\rangle$ to find $h \in \omega^{\omega}$ such that:

(a) $h$ is a.d. from $\mathscr{B}$.

(b) $\left\langle f_{\alpha}: \alpha<\kappa\right\rangle$ avoids $\mathscr{B} \cup\{h\}$.

(c) $\forall g \in \mathscr{G}_{\alpha}[|h \cap g|=\omega]$.

(d) $\exists \alpha<\kappa\left[\left|h \cap f_{\alpha}\right|<\omega\right]$.

Now, we can define $\mathscr{A}_{\alpha}=\mathscr{B} \cup\{h\}$. It is clear that $\mathscr{A}_{\alpha}$ is what is required.

The original motivation for the above result came from the following considerations. Under $\mathrm{CH}$, all MAD families have size $\aleph_{1}$. Hence any strongly MAD family is automatically very MAD. Given some such consequence of $\mathrm{CH}$, it is natural to ask whether this consequence also obtains under MA. So we originally wanted to know if under MA, all strongly MAD families are also very MAD. The above result shows that this fails badly.

Corollary 12. Assume $M A+\neg C H$. There is a strongly MAD family that is not very MAD.

We end this section with a conjecture. We do not know for which cardinals $\kappa$ there is an a.d. family $\mathscr{A} \subset \omega^{\omega}$ with $\operatorname{st}(\mathscr{A})=\kappa$ just on the basis of 
ZFC alone. In view of Lemma 6, the following conjecture is a natural generalization of our result that van Douwen MAD families exist on the basis of ZFC alone.

Conjecture 13. For every $n \in \omega$, there is an a.d. family $\mathscr{A} \subset \omega^{\omega}$ with $\operatorname{st}(\mathscr{A}) \geq n$.

3. A strongly MAD family from $\mathfrak{b}=\mathfrak{c}$. In this section we will construct a strongly MAD family from $\mathfrak{b}=\mathfrak{c}$. Kastermans [14] pointed out that the standard construction of a strongly MAD family from $\mathrm{MA}(\sigma$-centered $)$ actually yields a very MAD family. He asked if there is a different construction that distinguishes between strongly and very MAD families. This section is intended to address his question. The construction of a strongly MAD family given here cannot be used to build a very MAD family. This is because $\mathfrak{b}=\mathfrak{c}$ holds in the Laver model, where, as we will see in Section 4, there are no very MAD families. The question of whether strongly MAD families exist on the basis of ZFC alone remains open.

Hrušák [8], Kurilić [18] and Brendle and Yatabe [4] construct a Cohenindestructible MAD family of sets from $\mathfrak{b}=\mathfrak{c}$. Our construction was inspired by theirs, although our presentation is different. We will inductively construct a strongly MAD family in $\mathfrak{c}$ steps. At each step we will deal with a given countable family of functions. We will deal with this given collection by first forming an $(\omega, \kappa)$ gap consisting of infinite partial functions. We will then use $\mathfrak{b}=\mathfrak{c}$ to separate this gap by an infinite partial function.

Lemma 14. Assume $\mathfrak{b}=\mathfrak{c}$. Let $\mathscr{A} \subset \omega^{\omega}$ be an a.d. family with $|\mathscr{A}|<\mathfrak{c}$. Suppose $\left\{g_{n}: n \in \omega\right\} \subset \omega^{\omega}$ is a collection of functions avoiding $\mathscr{A}$. There is $h \in \omega^{\omega}$ such that:

(1) $\forall h^{\prime} \in \mathscr{A}\left[\left|h \cap h^{\prime}\right|<\omega\right]$.

(2) $\forall n \in \omega\left[\left|h \cap g_{n}\right|=\omega\right]$.

Proof. Firstly, observe that $\mathfrak{b}=\mathfrak{c}$ implies both $\mathfrak{a}=\mathfrak{c}$ and $\mathfrak{a}_{\mathfrak{e}}=\mathfrak{c}$. Now, for $n \in \omega$ consider $\mathscr{A} \cap g_{n}=\left\{h \cap g_{n}: h \in \mathscr{A} \wedge\left|h \cap g_{n}\right|=\omega\right\}$. This is an a.d. family on $g_{n}$. Since $g_{n}$ avoids $\mathscr{A}$ and since $|\mathscr{A}|<\mathfrak{c}$, it cannot be a MAD family on $g_{n}$. So we may find an infinite partial function $p_{n} \subset g_{n}$ which is a.d. from everything in $\mathscr{A}$. By refining their domains if necessary, we may assume that $\forall n<m<\omega\left[\operatorname{dom}\left(p_{n}\right) \cap \operatorname{dom}\left(p_{m}\right)=0\right]$. Now, $\left(\left\{p_{n}: n \in \omega\right\}, \mathscr{A}\right)$ is the gap we would like to separate using an infinite partial function. We will use the assumption $\mathfrak{b}=\mathfrak{c}$ to do this. Let $\lambda=|\mathscr{A}|$ and put $\mathscr{A}=\left\{h_{\alpha}: \alpha<\lambda\right\}$. Remember that $\lambda<\mathfrak{c}$. For each $\alpha<\lambda$, define a function $F_{\alpha} \in \omega^{\omega}$ as follows. For each $n \in \omega,\left\{k \in \operatorname{dom}\left(p_{n}\right): p_{n}(k)=h_{\alpha}(k)\right\}$ is finite. So, we can define $F_{\alpha}(n)=\max \left\{k \in \operatorname{dom}\left(p_{n}\right): p_{n}(k)=h_{\alpha}(k)\right\}$. Since we are assuming $\mathfrak{b}=\mathfrak{c}$, the family $\left\{F_{\alpha}: \alpha<\lambda\right\}$ is bounded. Choose a function $F \in \omega^{\omega}$ so that 
$\forall \alpha<\lambda\left[F_{\alpha}<{ }^{*} F\right]$. Define $p=\bigcup\left(p_{n} \backslash\left(p_{n} \mid F(n)\right)\right)$. Clearly, $p$ is an infinite partial function, and for all $n \in \omega,\left|p \cap g_{n}\right|=\omega$.

We will check that $\forall \alpha<\lambda\left[\left|p \cap h_{\alpha}\right|<\omega\right]$. Fix $\alpha<\lambda$. Suppose $k \in \operatorname{dom}(p)$ and $p(k)=h_{\alpha}(k)$. By our choice of the $p_{n}$, it follows that there is a unique $n$ such that $k \in \operatorname{dom}\left(p_{n}\right)$. Thus, $p_{n}(k)=h_{\alpha}(k)$, and so $k \leq F_{\alpha}(n)$. But since $k \in \operatorname{dom}(p)$, it follows that $k \geq F(n)$, whence $F(n) \leq F_{\alpha}(n)$. Thus $k \in \bigcup\left\{F_{\alpha}(n)+1: F(n) \leq F_{\alpha}(n)\right\}$, which is a finite set. So we conclude that $p \cap h_{\alpha}$ is finite.

Now, we are almost done. We just need to extend $p$ into a total function. We will use $\mathfrak{a}_{\mathfrak{e}}=\mathfrak{c}$ to do this. Let $X=\operatorname{dom}(p)$ and $Y=\omega \backslash X$. As $\mathscr{A}$ is an a.d. family in $\omega^{\omega}$ with $|\mathscr{A}|<\mathfrak{c}$, it is not maximal. Let $h_{0} \in \omega^{\omega}$ be a.d. from $\mathscr{A}$. Clearly, $h=\left(p \cup h_{0}\right) \uparrow Y$ is as needed.

TheOREm 15. Assume $\mathfrak{b}=\mathfrak{c}$. There is a strongly MAD family of size $\mathfrak{c}$.

Proof. We will build the strongly MAD family, $\mathscr{A}$, in $\mathfrak{c}$ steps. Since $\mathfrak{c}^{\omega}$ $=\mathfrak{c}$, we can let $\left\{\mathscr{G}_{\alpha}: \alpha<\mathfrak{c}\right\}$ enumerate all the countable subsets of $\omega^{\omega}$. We will build $\mathscr{A}$ as the union of an increasing sequence of a.d. families. We will build a sequence $\left\langle\mathscr{A}_{\alpha}: \alpha<\mathfrak{c}\right\rangle$ such that:

(1) $\mathscr{A}_{\alpha} \subset \omega^{\omega}$ is an a.d. family with $|\mathscr{A}| \leq|\alpha|$.

(2) If $\alpha<\beta<\mathfrak{c}$, then $\mathscr{A}_{\alpha} \subset \mathscr{A}_{\beta}$.

(3) If $\mathscr{G}_{\alpha}$ avoids $\bigcup\left\{\mathscr{A}_{\beta}: \beta<\alpha\right\}$, then $\exists h \in \mathscr{A}_{\alpha} \forall g \in \mathscr{G}_{\alpha}[|h \cap g|=\omega]$.

Assume that the sequence $\left\langle\mathscr{A}_{\beta}: \beta<\alpha\right\rangle$ has already been built. Set $\mathscr{B}=$ $\bigcup \mathscr{A}_{\beta}$. Then $\mathscr{B} \subset \omega^{\omega}$ is an a.d. family with $|\mathscr{B}|<\mathfrak{c}$. If $\mathscr{G}_{\alpha}$ does not avoid $\mathscr{B}$, then we can simply set $\mathscr{A}_{\alpha}=\mathscr{B}$. So we assume that $\mathscr{G}_{\alpha}$ avoids $\mathscr{B}$. Now, we may apply Lemma 14 with $\mathscr{B}$ as $\mathscr{A}$ and $\mathscr{G}_{\alpha}$ as $\left\{g_{n}: n \in \omega\right\}$ to find $h \in \omega^{\omega}$ such that $h$ is a.d. from $\mathscr{B}$ and $\forall g \in \mathscr{G}_{\alpha}[|h \cap g|=\omega]$. Then it is clear that $\mathscr{A}_{\alpha}=\mathscr{B} \cup\{h\}$ is as required.

We remark that even though we have not explicitly tried to ensure that $|\mathscr{A}|=\mathfrak{c}$, it is true because $\mathfrak{b}=\mathfrak{c}$ implies $\mathfrak{a}_{\mathfrak{e}}=\mathfrak{c}$.

COROLlaRY 16. There are strongly MAD families in the Laver and Hechler models.

As mentioned above, it is unknown if strongly MAD families always exist. We conjecture below that this is not the case. We will prove a partial result in this direction in Section 7, where we will show that it is consistent to have no "large" strongly MAD families (Theorem 75).

Conjecture 17. It is consistent to have no strongly MAD families.

4. Brendle's conjecture: consistency of no very MAD families. In this section we will show that if $\operatorname{cov}(\mathcal{M})<\mathfrak{a}_{\mathfrak{e}}$, then there are no very MAD families. This was conjectured by Brendle in an email to Kastermans. 
Kastermans showed that very MAD families exist under MA and asked if their existence can be proved in ZFC. Our result implies that there are no very MAD families in the Laver, Random or Blass-Shelah models. For the case of the Laver and Random models, this was already known to Brendle. Brendle also pointed out in the same email that his conjecture would imply that there are no very MAD families in a typical template model. Our proof uses the following characterization of $\operatorname{cov}(\mathcal{M})$.

THEOREM 18 (see [2] or [3]). The following are equivalent for a cardinal $\kappa \geq \omega:$

(1) The reals cannot be covered by $\kappa$ meager sets.

(2) If $\left\{f_{\alpha}: \alpha<\kappa\right\} \subset \omega^{\omega}$ is a collection of $\kappa$ functions, there is $h \in \omega^{\omega}$ such that $\forall \alpha<\kappa\left[\left|f_{\alpha} \cap h\right|=\omega\right]$.

Now, this characterization implies that there is a family $\left\{f_{\alpha}: \alpha<\right.$ $\operatorname{cov}(\mathcal{M})\} \subset \omega^{\omega}$ such that there is no $h \in \omega^{\omega}$ for which $\left|h \cap f_{\alpha}\right|=\omega$ holds for all $\alpha<\operatorname{cov}(\mathcal{M})$. Therefore, if this $\left\{f_{\alpha}: \alpha<\operatorname{cov}(\mathcal{M})\right\}$ avoids an a.d. family $\mathscr{A} \subset \omega^{\omega}$, and if $\operatorname{cov}(\mathcal{M})<|\mathscr{A}|$, then $\mathscr{A}$ cannot be a very MAD family. However, given an arbitrary very MAD family $\mathscr{A}$, there is no reason to expect the family $\left\{f_{\alpha}: \alpha<\operatorname{cov}(\mathcal{M})\right\}$ to avoid it. We deal with this by showing that in the above theorem one can replace functions with objects that are a bit "fatter", namely slaloms. This will provide a new characterization of the cardinal $\operatorname{cov}(\mathcal{M})$. Their "fatness" will ensure that the slaloms avoid any a.d. family.

Definition 19. A function $S: \omega \rightarrow[\omega]^{<\omega}$ is called a slalom if $\forall n \in \omega$ $\left[|S(n)| \leq 2^{n}\right]$. We say that $S$ is a wide slalom if $\forall n \in \omega\left[|S(n)|=2^{n}\right]$.

TheOREM 20. Let $\kappa$ be an infinite cardinal. The following are equivalent:

(1) The reals cannot be covered by $\kappa$ meager sets.

(2) If $\left\{S_{\alpha}: \alpha<\kappa\right\}$ is a collection of $\kappa$ wide slaloms, there is $h \in \omega^{\omega}$ such that $\forall \alpha<\kappa \exists^{\infty} n \in \omega\left[h(n) \in S_{\alpha}(n)\right]$.

Proof. $\neg(2) \Rightarrow \neg(1)$. Fix a family of wide slaloms $\left\{S_{\alpha}: \alpha<\kappa\right\}$ for which the consequent of (2) fails. For each $\alpha<\kappa$ set $E_{\alpha}=\left\{h \in \omega^{\omega}: \forall \forall^{\infty} n \in \omega\right.$ $\left.\left[h(n) \notin S_{\alpha}(n)\right]\right\}$. It is clear that each $E_{\alpha}$ is meager. Also, by assumption, $\omega^{\omega}=\bigcup E_{\alpha}$. Thus (1) is false.

$(2) \Rightarrow(1)$. Assume (2). We will show that clause (2) of Theorem 18 holds. Fix a family $\left\{f_{\alpha}: \alpha<\kappa\right\} \subset \omega^{\omega}$. Since we may code functions from $\omega$ to $[\omega \times \omega]^{<\omega}$ by slaloms, our assumption entails the following:

(*) For every family $\left\{H_{\alpha}: \alpha<\kappa\right\}$ of functions from $\omega$ to $[\omega \times \omega]<\omega$ such that $\forall n \in \omega\left[\left|H_{\alpha}(n)\right|=2^{n}\right]$, there is $g \in(\omega \times \omega)^{\omega}$ so that $\forall \alpha<\kappa$ $\exists^{\infty} n \in \omega\left[g(n) \in H_{\alpha}(n)\right]$. 
Now, for each $n \in \omega$ set $l_{n}=2^{n}-1$ and $I_{n}=\left[l_{n}, l_{n+1}\right)$. Thus $\left\langle I_{n}: n \in \omega\right\rangle$ is an interval partition of $\omega$ with $\left|I_{n}\right|=2^{n}$. Let us define a family $\left\{H_{\alpha}\right.$ : $\alpha<\kappa\}$ of functions from $\omega$ to $[\omega \times \omega]^{<\omega}$ by stipulating that for all $n \in \omega$, $H_{\alpha}(n)=f_{\alpha} \mid I_{n}$. Since $\left|I_{n}\right|=2^{n}, \forall n \in \omega\left[\left|H_{\alpha}(n)\right|=2^{n}\right]$. Therefore, by $(*)$ above, there is a $g \in(\omega \times \omega)^{\omega}$ so that

$$
\forall \alpha<\kappa \exists^{\infty} n \in \omega\left[g(n) \in H_{\alpha}(n)\right] .
$$

We may assume that $\forall n \in \omega\left[g(n) \in I_{n} \times \omega\right]$ because we can modify $g$ to make this true without affecting $(* *)$ above. Now, set $p=g^{\prime \prime} \omega$. It is clear that given our assumption about $g, p$ is an infinite partial function from $\omega$ to $\omega$. Now, let $h$ be a function in $\omega^{\omega}$ which extends $p$ (arbitrarily). We will check that $h$ is the function we are looking for.

Indeed, fix $\alpha<\kappa$. We must show that $\left|h \cap f_{\alpha}\right|=\omega$. We will prove that $\left|p \cap f_{\alpha}\right|=\omega$. For $n \in \omega$, let us use $\left\langle i_{n}, j_{n}\right\rangle$ to denote $g(n)$. Note that by our assumption on $g, \forall n \in \omega\left[i_{n} \in I_{n}\right]$. Also observe that by the definition of $p$, $\operatorname{dom}(p)=\left\{i_{n}: n \in \omega\right\}$ and $\forall n \in \omega\left[p\left(i_{n}\right)=j_{n}\right]$. By (**) above, the set $X=\left\{n \in \omega:\left\langle i_{n}, j_{n}\right\rangle \in H_{\alpha}(n)\right\}$ is infinite. By the definition of $H_{\alpha}$, it follows that $\forall n \in X\left[f_{\alpha}\left(i_{n}\right)=j_{n}=p\left(i_{n}\right)\right]$. Since the $I_{n}$ are disjoint, $\left\{i_{n}: n \in X\right\}$ is infinite, and so $\left|f_{\alpha} \cap p\right|=\omega$.

Lemma 21. Let $\mathscr{A} \subset \omega^{\omega}$ be a MAD family. Let $\lambda<\operatorname{st}(\mathscr{A})$ be a cardinal and let $\left\{S_{\alpha}: \alpha<\lambda\right\}$ be a family of wide slaloms. There is $h \in \mathscr{A}$ such that $\forall \alpha<\lambda \exists^{\infty} n \in \omega\left[h(n) \in S_{\alpha}(n)\right]$.

Proof. For each $\alpha<\lambda$, let $X_{\alpha}=\bigcup\left(\{n\} \times S_{\alpha}(n)\right)$. Observe that for any function $f \in \omega^{\omega}, \exists^{\infty} n \in \omega\left[f(n) \in S_{\alpha}(n)\right] \Leftrightarrow\left|X_{\alpha} \cap f\right|=\omega$. Hence, it suffices to produce $h \in \mathscr{A}$ such that $\forall \alpha<\lambda\left[\left|h \cap X_{\alpha}\right|=\omega\right]$. For each $\alpha<\lambda$, we will produce a total function $f^{\alpha} \subset X_{\alpha}$ avoiding $\mathscr{A}$. Notice that we can ensure that $f^{\alpha}$ avoids $\mathscr{A}$ by ensuring that it has infinite intersection with infinitely many members of $\mathscr{A}$. Indeed, given any finite collection of functions $\left\{f_{0}, \ldots, f_{n}\right\} \subset \omega^{\omega}$, there is a total function $g \subset X_{\alpha}$ which is a.d. from $f_{0}, \ldots, f_{n}$. This is because $S_{\alpha}$ is a wide slalom. But we are assuming that $\mathscr{A}$ is MAD. It now follows that for each $\alpha<\lambda$ there must be an infinite collection $\left\{h_{i}^{\alpha}: i \in \omega\right\} \subset \mathscr{A}$ such that $\forall i \in \omega\left[\left|h_{i}^{\alpha} \cap X_{\alpha}\right|=\omega\right]$. For each $i \in \omega$, set $p_{i}^{\alpha}=h_{i}^{\alpha} \cap X_{\alpha}$. Then $p_{i}^{\alpha}$ is an infinite partial function contained in $X_{\alpha}$. By refining their domains, it is possible to choose a collection of infinite partial functions $\left\{g_{i}^{\alpha}: i \in \omega\right\}$ such that $\forall i \in \omega\left[g_{i}^{\alpha} \subset p_{i}^{\alpha}\right]$ and $\forall i<j<\omega\left[\operatorname{dom}\left(g_{i}^{\alpha}\right) \cap \operatorname{dom}\left(g_{j}^{\alpha}\right)=0\right]$. Now, we can find a function $f^{\alpha} \in \omega^{\omega}$ with $\bigcup_{i \in \omega} g_{i}^{\alpha} \subset f^{\alpha} \subset X_{\alpha}$. Now, $\lambda<\operatorname{st}(\mathscr{A})$. So there is $h \in \mathscr{A}$ such that $\forall \alpha<\lambda\left[\left|h \cap f^{\alpha}\right|=\omega\right]$. This $h$ is the function we are looking for.

Theorem 22 (Brendle's Conjecture). If $\mathscr{A}$ is a very MAD family, then $|\mathscr{A}| \leq \operatorname{cov}(\mathcal{M})$. In particular, if $\operatorname{cov}(\mathcal{M})<\mathfrak{a}_{\mathfrak{e}}$, then there are no very $M A D$ families. 
Proof. Suppose, for a contradiction, that $\operatorname{cov}(\mathcal{M})<|\mathscr{A}|$. By Theorem 20, there is a family $\left\{S_{\alpha}: \alpha<\operatorname{cov}(\mathcal{M})\right\}$ of wide slaloms such that for every $h \in \omega^{\omega}$ there is $\alpha<\operatorname{cov}(\mathcal{M})$ such that $\forall^{\infty} n \in \omega\left[h(n) \notin S_{\alpha}(n)\right]$. But now, since $\operatorname{cov}(\mathcal{M})<|\mathscr{A}| \leq \operatorname{st}(\mathscr{A})$, we can apply Lemma 21 to get a function $h \in \mathscr{A}$ that contradicts this.

COROLlary 23. There are no very MAD families in the Laver, Random or Blass-Shelah models.

Proof. It is well-known (see [3]) that none of these forcings, nor their respective iterations, add Cohen reals. Thus in all of these models $\operatorname{cov}(\mathcal{M})$ $=\aleph_{1}$. On the other hand, each of these forcings makes the ground model meager. Hence in all three of these models non $(\mathcal{M})$, and hence $\mathfrak{a}_{\mathfrak{e}}$, is $\aleph_{2}$.

RemarK 24. Let $\mathfrak{a}_{\mathfrak{v}}$ be the least size of a van Douwen MAD family. Since very MAD families are van Douwen MAD, Theorem 22 implies that there are no very MAD families as long as $\operatorname{cov}(\mathcal{M})<\mathfrak{a}_{\mathfrak{v}}$. It is conceivable that $\mathfrak{a}_{\mathfrak{e}}<\mathfrak{a}_{\mathfrak{v}}$ is consistent, but no models of this are known.

In Section 2 we promised to give a proof that $\operatorname{st}(\mathscr{A}) \leq \operatorname{cov}(\mathcal{M})$ in this section. We end this section by fulfilling this promise.

Corollary 25. Let $\mathscr{A} \subset \omega^{\omega}$ be an a.d. family. Then $\operatorname{st}(\mathscr{A}) \leq \operatorname{cov}(\mathcal{M})$.

Proof. Suppose for a contradiction that $\operatorname{st}(\mathscr{A})>\operatorname{cov}(\mathcal{M})$. By Lemma 6 , $\mathscr{A}$ is van Douwen MAD. But now, we can argue just as in Theorem 22 to get a contradiction using Lemma 21.

5. Indestructibility properties of strongly MAD families. In this section we will study the effect of forcing on strongly MAD families. In particular, we will be interested in showing that certain posets preserve strongly MAD families.

We will assume familiarity with the basic theory of proper forcing. The reader may consult Abraham [1], Goldstern [7] or Shelah [23] for an introduction.

Definition 26. Let $\mathbb{P}$ be a notion of forcing and let $\mathscr{A} \subset \omega^{\omega}$ be a strongly MAD family. We will say that $\mathscr{A}$ is $\mathbb{P}$-indestructible if $\vdash_{\mathbb{P}} \mathscr{A}$ is MAD. We will say that $\mathscr{A}$ is strongly $\mathbb{P}$-indestructible if $\Vdash_{\mathbb{P}} \mathscr{A}$ is strongly MAD.

Brendle and Yatabe [4] have studied $\mathbb{P}$-indestructibility of MAD families of subsets of $\omega$ for various posets $\mathbb{P}$. The focus of their work was to provide combinatorial characterizations of the property of being a $\mathbb{P}$-indestructible MAD family of sets for some well known posets $\mathbb{P}$. Here our focus is instead to find those posets $\mathbb{P}$ for which strongly MAD families of functions are strongly $\mathbb{P}$-indestructible. 
If $\mathbb{P}$ is a poset which turns the ground model reals into a meager set, then it is clear that no MAD family $\mathscr{A} \subset \omega^{\omega}$ can be $\mathbb{P}$-indestructible. This is because any such poset adds an element of $\omega^{\omega}$ which is eventually different from all the elements of $\omega^{\omega}$ in the ground model (see [3]). Our intuition is that strongly MAD families are "so large" that a converse to this observation must be true, at least for "nicely" definable posets, meaning that such a poset can kill a strongly MAD family only by introducing an eventually different real. If we take "nicely" definable to mean Suslin proper (for example, see Section 7 of [7]), then the property of not turning the ground model meager is known to be equivalent to the seemingly stronger property of preserving Cohen reals over elementary submodels.

Definition 27. Let $\mathbb{P}$ be a poset. We say that $\mathbb{P}$ preserves $\sqsubseteq_{\mathbf{C}}$ if the following holds. For every $M \prec H(\theta), M$ countable, with $\mathbb{P} \in M$, whenever $p \in \mathbb{P} \cap M$ and $x$ is a Cohen real over $M$, there is $q \leq p$ which is $(M, \mathbb{P})$ generic such that $q \Vdash x$ is a Cohen real over $M[\stackrel{G}{G}]$.

It is easy to see that if $\mathbb{P}$ preserves $\sqsubseteq_{\mathbf{C}}$, then $\mathbb{P}$ does not make the ground model reals into a meager set. Shelah (see [23, Chapter 18]) showed that the converse is also true for Suslin proper posets. While a poset that preserves $\sqsubseteq_{\mathbf{C}}$ need not be Suslin proper, we believe that just preserving $\sqsubseteq_{\mathbf{C}}$ is good enough to guarantee that strongly MAD families are strongly indestructible for that poset. In fact, our official conjecture will be slightly stronger. We will introduce a property stronger than strong indestructibility, a property which, like the property of preserving $\sqsubseteq_{\mathbf{C}}$, is preserved by countable support iterations of proper forcings. We are ultimately interested not only in treating indestructibility for single step forcing extensions, but also for countable support iterations. We are unable to show that every strongly MAD $\mathscr{A}$ is strongly indestructible for any countable support iteration of proper posets for which $\mathscr{A}$ is strongly indestructible. However, we are able to prove the preservation of this slightly stronger property, which we next introduce, and which can be thought of as the property of preserving $\sqsubseteq$ c "relativized to $\mathscr{A}$ ".

Definition 28. Let $\mathscr{A} \subset \omega^{\omega}$ be an a.d. family. Let $M \prec H(\theta)$ be countable with $\mathscr{A} \in M$. We say that $h \in \mathscr{A}$ covers $M$ with respect to $\mathscr{A}$ if whenever $f \in M$ is an infinite partial function avoiding $\mathscr{A},|h \cap f|=\omega$.

Notice that by Lemma 7 , if $\mathscr{A} \subset \omega^{\omega}$ is a strongly MAD family and $M \prec H(\theta)$ is countable, then there is $h \in \mathscr{A}$ which covers $M$ with respect to $\mathscr{A}$.

Definition 29. Let $\mathscr{A} \subset \omega^{\omega}$ be a strongly MAD family and let $\mathbb{P}$ be a poset. Let $M \prec H(\theta)$ be countable with $\mathscr{A}, \mathbb{P} \in M$. We say that $\mathbf{R}(\mathscr{A}, \mathbb{P}, M)$ holds if whenever $p$ is a condition in $\mathbb{P} \cap M$ and $h \in \mathscr{A}$ covers $M$ with respect to $\mathscr{A}$, there is $q \leq p$ which is $(M, \mathbb{P})$ generic such that 
$q \Vdash h$ covers $M[\stackrel{\circ}{G}]$ with respect to $\mathscr{A}$. We say that $\mathbb{P}$ strongly preserves $\mathscr{A}$ if for every $M \prec H(\theta), M$ countable, with $\mathscr{A}, \mathbb{P} \in M, \mathbf{R}(\mathscr{A}, \mathbb{P}, M)$ holds.

Conjecture 30. Let $\mathscr{A} \subset \omega^{\omega}$ be a strongly MAD family and let $\mathbb{P}$ be a poset that preserves $\sqsubseteq_{\mathbf{C}}$. Then $\mathscr{A}$ is strongly $\mathbb{P}$-indestructible. Moreover, $\mathbb{P}$ strongly preserves $\mathscr{A}$.

Observe that Conjecture 30 implies that a Suslin proper poset fails to strongly preserve a strongly MAD family iff it adds an eventually different real. If true, our conjecture would say that a poset $\mathbb{P}$ either destroys all MAD families in the ground model, or else every strongly MAD family is strongly $\mathbb{P}$-indestructible, at least for the case of Suslin proper $\mathbb{P}$.

While we do not have a proof of Conjecture 30, we provide some good evidence for it in this section. In particular, we will show that any poset for which a certain kind of fusion argument can be carried out strongly preserves all strongly MAD families (Theorem 52). The class of posets for which this type of fusion can be done includes all the usual posets preserving $\sqsubseteq_{\mathbf{C}}$ that we are aware of, like Sacks, Miller and Silver forcing. In fact, for this class of posets, the same kind of fusion argument shows both the strong preservation of strongly MAD families as well as the preservation of $\sqsubseteq_{\mathbf{C}}$.

REMARK 31. Notice that if $\mathscr{A} \subset \omega^{\omega}$ is a strongly MAD family and if $\mathbb{P}$ is a poset which strongly preserves $\mathscr{A}$, then $\mathbb{P}$ is proper.

Our definition of strongly preserving requires $\mathbf{R}(\mathscr{A}, \mathbb{P}, M)$ to hold for all elementary submodels containing $\mathscr{A}$ and $\mathbb{P}$. But as is usual in the theory of proper forcing, it is sufficient if this is true for a club of such elementary submodels. We will prove this next, and in what follows, we will use this fact without further comment.

Lemma 32. Let $\mathscr{A} \subset \omega^{\omega}$ be a strongly $M A D$ family and let $\mathbb{P}$ be a poset. If $\{M \prec H(\theta):|M|=\omega \wedge \mathscr{A}, \mathbb{P} \in M \wedge \mathbf{R}(\mathscr{A}, \mathbb{P}, M)$ holds $\}$ contains a club in $[H(\theta)]^{\omega}$, then $\mathbb{P}$ strongly preserves $\mathscr{A}$.

Proof. Arguments of this sort are standard in the theory of properness; so we merely outline the steps. We must find a set $X \in H(\theta)$ that "captures" all the information necessary for deciding the truth of $\mathbf{R}(\mathscr{A}, \mathbb{P}, M)$ for any $M$ with $\mathscr{A}, \mathbb{P} \in M$. Let $\mathcal{F}_{\mathscr{A}}=\{f: f$ is an infinite partial function avoiding $\mathscr{A}\}$ and let $A_{\mathbb{P}}=\left\{\stackrel{\circ}{x} \in \mathbf{V}^{\mathbb{P}}: \stackrel{\circ}{x}\right.$ is a nice $\mathbb{P}$ name for a subset of $\left.\omega \times \omega\right\}$. Put $X=\mathbb{P} \cup \mathcal{P}(\mathbb{P}) \cup A_{\mathbb{P}} \cup \mathscr{A} \cup \mathcal{F}_{\mathscr{A}}$. We will argue that this $X$ does the job. Notice that if $\mathcal{M} \prec H(\theta)$ is countable with $\mathscr{A}, \mathbb{P} \in M$, then $X$, and hence $[X]^{\omega}$, are elements of $M$. Now, given $a \in[X]^{\omega}$ and $q \in \mathbb{P}$, say that $q$ is $(a, \mathbb{P})$ generic if whenever $D \subset \mathbb{P}$ is a dense open set in $a, q \Vdash a \cap D \cap \dot{G} \neq 0$. Similarly, say that $h \in \mathscr{A}$ covers a with respect to $\mathscr{A}$ if $|h \cap f|=\omega$ for all $f \in a \cap \mathcal{F}_{\mathscr{A}}$. Finally, say that $a \in[X]^{\omega}$ is good if whenever $p \in \mathbb{P} \cap a$ and $h \in \mathscr{A}$ covers $a$ with respect to $\mathscr{A}$, there is $q \leq p$ which is $(a, \mathbb{P})$ generic such 
that $q \Vdash h$ covers $a[\stackrel{\circ}{G}]$ with respect to $A$. It is easy to see that if $M \prec H(\theta)$ is countable with $\mathscr{A}, \mathbb{P} \in M$ and if $\mathbf{R}(\mathscr{A}, \mathbb{P}, M)$ holds, then $M \cap X$ is good. Thus our assumption implies that $C=\left\{a \in[X]^{\omega}: a\right.$ is good $\}$ contains a club in $[X]^{\omega}$. Now, fix a countable $M \prec H(\theta)$ with $\mathscr{A}, \mathbb{P} \in M$. We must show that $\mathbf{R}(\mathscr{A}, \mathbb{P}, M)$ holds. Notice that $C \in M$ and since $C$ contains a club, $X \cap M \in C$. Therefore, $X \cap M$ is good. Now, fix $p \in \mathbb{P} \cap M$ and let $h \in \mathscr{A}$ cover $M$ with respect to $\mathscr{A}$. Obviously, $p \in \mathbb{P} \cap X \cap M$ and $h$ covers $X \cap M$ with respect to $\mathscr{A}$. So, we can find $q \leq p$ which is $(X \cap M, \mathbb{P})$ generic such that $q \Vdash h$ covers $(X \cap M)[\stackrel{\circ}{G}]$ with respect to $\mathscr{A}$. It is easily seen that $q$ is in fact $(M, \mathbb{P})$ generic. We will argue that $q \Vdash h$ covers $M[G]$ with respect to $\mathscr{A}$. Indeed, let $G$ be a $(\mathbf{V}, \mathbb{P})$ generic filter with $q \in G$ and suppose $f \in M[G]$ is an infinite partial function avoiding $\mathscr{A}$. By elementarity of $M$, there is $\stackrel{\circ}{x} \in A_{\mathbb{P}} \cap M$ such that $\stackrel{x}{x}[G]=f$. But then $\stackrel{\circ}{x} \in X \cap M$, and so $f \in(X \cap M)[G]$. Therefore, $|h \cap f|=\omega$, and we are done.

Lemma 33. Let $\mathscr{A} \subset \omega^{\omega}$ be a strongly $M A D$ family and let $\mathbb{P}$ be a poset that strongly preserves $\mathscr{A}$. Then $\mathscr{A}$ is strongly $\mathbb{P}$-indestructible.

Proof. Firstly, note that if $\mathscr{A}$ is strongly MAD and $M$ is a countable elementary submodel, then, by Lemma 7 , there is $h \in \mathscr{A}$ which covers $M$ with respect to $\mathscr{A}$. Suppose for a contradiction that $\mathscr{A}$ is not strongly $\mathbb{P}$ indestructible. Fix $M \prec H(\theta)$ with $|M|=\omega$ and $\mathbb{P}, \mathscr{A} \in M$. Now, by our assumption, we can find a set of $\mathbb{P}$-names $\left\{f_{i}: i \in \omega\right\} \in M$ and $p \in \mathbb{P} \cap M$ such that:

(1) $\forall i \in \omega\left[p \Vdash \AA_{i} \in \omega^{\omega} \wedge \stackrel{\circ}{f}_{i}\right.$ avoids $\left.\mathscr{A}\right]$.

(2) $\forall h \in \mathscr{A}\left[p \Vdash \exists i \in \omega\left[\left|h \cap \dot{\circ}_{i}\right|<\omega\right]\right]$.

Now, fix $h \in \mathscr{A}$ which covers $M$ with respect to $\mathscr{A}$. Choose $q \leq p$ such that $q \Vdash h$ covers $M[\stackrel{\circ}{G}]$ with respect to $\mathscr{A}$. By elementarity, $\left\{\dot{f}_{i}: i \in \omega\right\} \subset M$. So, for each $i \in \omega, q \Vdash \AA_{i}^{\circ} \in \omega^{\omega} \cap M\left[\stackrel{G}{G} \wedge \wedge \stackrel{\circ}{f}_{i}\right.$ avoids $\mathscr{A}$. But then, for each $i \in \omega, q \Vdash\left|h \cap \stackrel{\circ}{f}_{i}\right|=\omega$, contradicting (2) above.

Our aim in the rest of this section will be to show that a large class of posets not turning the ground model reals into a meager set strongly preserve all strongly MAD families. As a warm up, we first show that the Cohen poset strongly preserves all strongly MAD families. Kurilić [18] and Hrušák and García Ferreira [9] showed that a strongly MAD family in $[\omega]^{\omega}$ (see Definition 70) stays maximal after Cohen forcing. While it is possible to modify their proof to get a proof of Theorem 36, we give a different proof which foreshadows the proof of the more general Theorem 52 .

The following lemma will play an important role in all our proofs of strong preservation. It allows us to transfer the property of avoiding $\mathscr{A}$ from a $\mathbb{P}$ name for a partial function to some partial function in the ground model. 
We will use clause (2) of the lemma below to ensure that certain partial functions we construct in the ground model from some $\mathbb{P}$ names avoid $\mathscr{A}$.

Lemma 34. Let $\mathscr{A} \subset \omega^{\omega}$ be an infinite a.d. family and let $\mathbb{P}$ be any poset. Suppose $\stackrel{f}{f}$ is a $\mathbb{P}$ name such that $\Vdash \dot{f}$ is an infinite partial function avoiding $\mathscr{A}$. There is a countable set of $\mathbb{P}$ names $\left\{f_{i}: i \in \omega\right\}$ such that:

(1) $\Vdash \dot{\circ}_{i} \subset \dot{f}$ is an infinite partial function.

(2) $\Vdash \forall g \subset \omega \times \omega\left[\right.$ if $\forall i \in \omega\left[\left|g \cap \stackrel{\circ}{i}_{i}\right|=\omega\right]$, then $g$ avoids $\left.\mathscr{A}\right]$.

Proof. Let $G$ be any $(\mathbf{V}, \mathbb{P})$ generic filter. We will work inside $\mathbf{V}[G]$. By assumption, $f[G]$ is an infinite partial function avoiding $\mathscr{A}$. We will find a countable set $\left\{f_{i}: i \in \omega\right\}$ of infinite partial subfunctions of $f[G]$ such that any $g \subset \omega \times \omega$ having infinite intersection with all the $f_{i}$ avoids $\mathscr{A}$. Consider $\mathscr{A} \cap f[G]=\{h \cap \AA[G]: h \in \mathscr{A} \wedge|h \cap \dot{f}[G]|=\omega\}$. This is an a.d. family on $f[G]$. The proof will break into two cases depending on whether $\mathscr{A} \cap f[G]$ is finite or infinite.

First, consider the case when $\mathscr{A} \cap f[G]$ is finite. Since $f[G]$ avoids $\mathscr{A}$, we can find an infinite partial function $f_{0} \subset \dot{f}[G]$ that is a.d. from $\mathscr{A}$. Now, for each $i \in \omega$, we can simply set $f_{i}$ equal to $f_{0}$. We will check that this will do. Indeed, suppose $g \subset \omega \times \omega$ has infinite intersection with $f_{0}$. If $g$ did not avoid $\mathscr{A}$, then since $f_{0}$ is a.d. from $\mathscr{A}, f_{0}$ would also be a.d. from $g$. Therefore, $g$ avoids $\mathscr{A}$.

Next, suppose that $\mathscr{A} \cap \AA[G]$ is infinite. Choose an infinite set $\left\{h_{i}: i \in \omega\right\}$ $\subset \mathscr{A}$ such that $\forall i \in \omega\left[\left|h_{i} \cap \dot{f}[G]\right|=\omega\right]$. Now, for each $i \in \omega$ set $f_{i}$ equal to $h_{i} \cap f[G]$. Thus $f_{i}$ is an infinite partial subfunction of $f[G]$. Now, suppose $g \subset \omega \times \omega$ has infinite intersection with all the $f_{i}$. Clearly, then, $\left|g \cap h_{i}\right|=\omega$, for all $i \in \omega$. Since $g$ has infinite intersection with infinitely many members of $\mathscr{A}$, it avoids $\mathscr{A}$.

Now, back in the ground model $\mathbf{V}$, since $G$ was an arbitrary $(\mathbf{V}, \mathbb{P})$ generic filter, we can use the maximal principle to find a countable set of names $\left\{f_{i}: i \in \omega\right\}$ which are forced to have the same properties as $\left\{f_{i}: i \in \omega\right\}$ defined above.

Lemma 35. Let $\mathbb{P}=\operatorname{Fn}(\omega, 2)$. Let $\stackrel{\circ}{f}$ be a name and suppose that $\Vdash \stackrel{\circ}{f}$ is an infinite partial function. Suppose that $\left\{\AA_{i}^{\circ}: i \in \omega\right\}$ is a set of names so that for each $i \in \omega, \Vdash \dot{f}_{i} \subset \dot{f}$ is an infinite partial function. Let $p \in \mathbb{P}$. There is an infinite partial function $g$ such that

(1) $\forall i \in \omega\left[p \Vdash\left|g \cap \stackrel{\circ}{f}_{i}\right|=\omega\right]$.

(2) $\forall n \in \operatorname{dom}(g) \exists q \leq p[q \Vdash n \in \operatorname{dom}(\stackrel{\circ}{f}) \wedge \stackrel{\circ}{f}(n)=g(n)]$.

Proof. Let $\left\{q_{j}: j \in \omega\right\}$ enumerate $\{q \in \mathbb{P}: q \leq p\}$. We will build $g$ by induction as the union of an increasing sequence of finite partial functions $g_{j}$. We will build a sequence $\left\langle g_{j}:-1 \leq j<\omega\right\rangle$ such that: 
(a) $g_{-1}=0$ and $g_{j-1} \subset g_{j}$ is a finite partial function.

(b) $\exists q \leq q_{j} \forall i \leq j \exists k_{j}^{i}>j \exists m_{j}^{i} \in \omega\left[q \Vdash k_{j}^{i} \in \operatorname{dom}\left(\stackrel{\circ}{f}_{i}\right) \wedge \stackrel{\circ}{f}_{i}\left(k_{j}^{i}\right)=m_{j}^{i}\right]$.

(c) $g_{j}=g_{j-1} \cup\left\{\left\langle k_{j}^{i}, m_{j}^{i}\right\rangle: i \leq j\right\}$.

We will first argue that $g=\bigcup g_{j}$ will satisfy requirements (1) and (2) above. To see that (1) holds, suppose for a contradiction that for some $i \in \omega$, there is a $p_{0} \leq p$ and $k \in \omega$ such that $p_{0} \Vdash \forall n>k\left[n \in \operatorname{dom}\left(\stackrel{\circ}{f}_{i}\right) \cap \operatorname{dom}(g) \Rightarrow\right.$ $\left.\AA_{i}(n) \neq g(n)\right]$. There are infinitely many conditions below $p_{0}$. So it is possible to find $j \geq k, i$ such that $q_{j} \leq p_{0}$. But then by clause (b) and (c), there is a $q \leq q_{j}$ and numbers $k_{j}^{i}>j \geq k$ and $m_{j}^{i} \in \omega$ such that $k_{j}^{i} \in \operatorname{dom}(g)$, $g\left(k_{j}^{i}\right)=m_{j}^{i}$, and $q \Vdash k_{j}^{i} \in \operatorname{dom}\left(\stackrel{\circ}{i}_{i}\right) \wedge \stackrel{\circ}{f}_{i}\left(k_{j}^{i}\right)=m_{j}^{i}$, which is a contradiction. Next, to see that (2) holds, suppose that $n \in \operatorname{dom}(g)$. By clause (c) above, $n=k_{j}^{i}$ for some $i \leq j$, and $g(n)=m_{j}^{i}$. But then by clause (b), there is a $q \leq q_{j} \leq p$ such that $q \Vdash n \in \operatorname{dom}\left(\stackrel{\circ}{f}_{i}\right) \wedge \stackrel{\circ}{f}_{i}(n)=m_{j}^{i}=g(n)$. Since $\stackrel{\circ}{f}_{i}$ is forced to be a subfunction of $\stackrel{\circ}{f}$, we have $q \Vdash n \in \operatorname{dom}(\stackrel{\circ}{f}) \wedge \stackrel{\circ}{f}(n)=g(n)$, which is as required.

Now, let us build the sequence $\left\langle g_{j}:-1 \leq j<\omega\right\rangle$. At stage $j$, suppose that $g_{j-1}$ is given to us. As all the $f_{i}$ are forced to be infinite partial functions, we can successively extend $q_{j} j+1$ times to find a condition $q \leq q_{j}$ and numbers $\max \left\{\max \left(\operatorname{dom}\left(g_{j-1}\right)\right), j\right\}<k_{j}^{0}<\cdots<k_{j}^{j}$ and $m_{j}^{0}, \ldots, m_{j}^{j} \in \omega$ such that $\forall i \leq j\left[q \Vdash k_{j}^{i} \in \operatorname{dom}\left(\dot{f}_{i}\right) \wedge \stackrel{\circ}{f}_{i}\left(k_{j}^{i}\right)=m_{j}^{i}\right]$. Since the $k_{j}^{i}$ are different for different values of $i$, we can set $g_{j}=g_{j-1} \cup\left\{\left\langle k_{j}^{0}, m_{j}^{0}\right\rangle, \ldots,\left\langle k_{j}^{j}, m_{j}^{j}\right\rangle\right\}$. It is clear that $g_{j}$ satisfies conditions (a)-(c).

Theorem 36. Let $\mathbb{P}=\operatorname{Fn}(\omega, 2)$. If $\mathscr{A} \subset \omega^{\omega}$ is a strongly MAD family, then $\mathbb{P}$ strongly preserves $\mathscr{A}$.

Proof. Let $\mathscr{A} \subset \omega^{\omega}$ be a strongly MAD family. Fix a countable elementary submodel $M \prec H(\theta)$ with $\mathbb{P}, \mathscr{A} \in M$. Choose $h \in \mathscr{A}$ which covers $M$ with respect to $\mathscr{A}$, and let $p \in \mathbb{P} \cap M$ be any condition. It is well known that $p$ is always $(M, \mathbb{P})$ generic. We will argue that $p \Vdash h$ covers $M[G]$ with respect to $\mathscr{A}$.

Suppose for a contradiction that there are $q \leq p, \stackrel{\circ}{f} \in M \cap \mathbf{V}^{\mathbb{P}}$ and $n \in \omega$ such that:

(*) $\Vdash f$ is an infinite partial function avoiding $\mathscr{A}$.

$(* *) q \Vdash \forall m>n[m \in \operatorname{dom}(\stackrel{\circ}{f}) \Rightarrow \stackrel{\circ}{f}(m) \neq h(m)]$.

Since $\mathbb{P}$ is countable, $\mathbb{P} \subset M$. Therefore, $q \in M$. Now, we can apply Lemma 34 to $f$ to find a countable set of names $\left\{f_{i}^{\circ}: i \in \omega\right\} \in M$ that satisfy clauses (1) and (2) of Lemma 34. Clause (1) of Lemma 34 implies that $f$ and $\left\{f_{i}^{\circ}: i \in \omega\right\}$ satisfy the hypothesis of Lemma 35. Thus we can apply Lemma 35 with the condition $q$ in place of $p$ to find an infinite partial 
function $g \in M$ which satisfies clauses (1) and (2) of Lemma 35 (with respect to $q$ ). Now, clause (2) of Lemma 34 and clause (1) of Lemma 35 together imply that $g$ avoids $\mathscr{A}$. As $h$ covers $M$ with respect to $\mathscr{A},|h \cap g|=\omega$. Choose $n<m \in \operatorname{dom}(g)$ such that $g(m)=h(m)$. But by clause (2) of Lemma 35, there is $r \leq q$ such that $r \Vdash m \in \operatorname{dom}(f) \wedge \stackrel{\circ}{f}(m)=g(m)=h(m)$, which contradicts $(* *)$ above.

Corollary 37. Let $\mathscr{A} \subset \omega^{\omega}$ be a strongly $M A D$ family. Let $P=\mathrm{Fn}(\omega, 2)$. Then $\mathscr{A}$ is $\mathbb{P}$-indestructible. In fact, $\mathscr{A}$ is strongly $\mathbb{P}$-indestructible.

An immediate consequence of the Cohen-indestructibility of strongly MAD families is a strengthening of a result of Steprāns [15] which says that strongly MAD families cannot be analytic.

Corollary 38. If $\mathscr{A} \subset \omega^{\omega}$ is strongly $M A D$, then $\mathscr{A}$ does not contain perfect sets.

Proof. Suppose for a contradiction that $T \subset \omega^{<\omega}$ is a perfect tree such that $[T] \subset \mathscr{A}$. Let $P=\operatorname{Fn}(\omega, 2)$ be Cohen forcing and $G$ be a $(\mathbf{V}, \mathbb{P})$ generic filter. Since $T$ is perfect, $[T]$ has a new branch in $\mathbf{V}[G]$. That is, there is a $b \in[T] \cap \mathbf{V}[G]$, which is not a member of $\mathbf{V}$. We will argue that $b$ is a.d. from $\mathscr{A}$, contradicting the Cohen-indestructibility of $\mathscr{A}$. First of all, notice that in $\mathbf{V}$, the following statement is true: any two distinct branches through $T$ are a.d. This statement is $\Pi_{1}^{1}$ and hence absolute. So it is still true in $\mathbf{V}[G]$ that any two distinct branches through $T$ are a.d. In particular, $b$ is a.d. from $[T] \cap \mathbf{V}$. Next, suppose that $f \in \mathscr{A} \backslash([T] \cap \mathbf{V})$. Notice that in $\mathbf{V}$, the following statement holds: $f$ is a.d. from every branch through $T$. This is again $\Pi_{1}^{1}$, and hence absolute. Thus in $\mathbf{V}[G]$ every branch through $T$ is a.d. from $f$. In particular, $b$ is a.d. from $f$, and we are done.

We now continue with our demonstration that a certain large class of forcings not making the ground model reals meager strongly preserve strongly MAD families. We adopt a general framework and show that all forcings for which a certain type of fusion argument can be carried out (Definition 40) have this property. But before doing this, we will first check that the required type of fusion argument can be done for Sacks and Miller forcing. The conditions in our definition might seem technical, but they are a natural abstraction of the properties of these two forcings. This will be clear from the proof of Theorem 48.

Definition 39. Let $(\mathbb{P}, \leq)$ be a poset. We say that $(\mathbb{P}, \leq)$ has fusion if there is a sequence $\left\langle\leq_{n}: n \in \omega\right\rangle$ of partial orderings on $\mathbb{P}$ such that:

(1) $\forall p, q \in \mathbb{P}\left[q \leq_{n} p \Rightarrow q \leq_{n-1} p\right]$, with $\leq_{-1}$ being $\leq$.

(2) If $\left\langle p_{n}: n \in \omega\right\rangle$ is a sequence with $p_{n+1} \leq_{n} p_{n}$, then $\exists q \in \mathbb{P} \forall n \in \omega$ $\left[q \leq_{n} p_{n}\right]$. 
Definition 40. Let $\mathbb{P}$ be a poset. We say that $\mathbb{P}$ has diagonal fusion if there exist a sequence $\left\langle\leq_{n}: n \in \omega\right\rangle$ of partial orderings on $\mathbb{P}$, a strictly increasing sequence of natural numbers $\left\langle i_{n}: n \in \omega\right\rangle$ with $i_{0}=0$, and for each $p \in \mathbb{P}$ a sequence $I_{p}=\left\langle p_{i}: i \in \omega\right\rangle \in \mathbb{P}^{\omega}$ such that the following hold:

(1) $\mathbb{P}$ has fusion with respect to $\left\langle\leq_{n}: n \in \omega\right\rangle$.

(2) $\forall i \in \omega\left[p_{i} \leq p\right]$.

(3) If $q \leq p$, then $\exists^{\infty} i \in \omega\left[q \not \perp p_{i}\right]$.

(4) If $q \leq_{n} p$, then $\forall i<i_{n}\left[q_{i} \leq p_{i}\right]$, where $I_{q}=\left\langle q_{i}: i \in \omega\right\rangle$.

(5) If $\left\langle r_{i}: i_{n} \leq i<i_{n+1}\right\rangle$ is a sequence such that $\forall i \in\left[i_{n}, i_{n+1}\right)\left[r_{i} \leq p_{i}\right]$, then $\exists q \leq_{n} p \forall i \in\left[i_{n}, i_{n+1}\right)\left[q_{i} \leq r_{i}\right]$, where $I_{q}=\left\langle q_{i}: i \in \omega\right\rangle$.

Our terminology is motivated by analogy with Miller forcing, where the notion of diagonal fusion across a Miller tree occurs. We show in Theorem 48 below that conditions (1)-(5) are abstractions of what goes on in the case of diagonal fusion through a Miller tree. In the case of Miller forcing, $I_{T}$, as a set, is just the collection of all subtrees of $T$ that correspond to the successors of its split nodes. Condition (5) corresponds to amalgamating extensions of these into the tree $T$.

Intuitively, posets with diagonal fusion are those Axiom A posets which allow for fusion arguments involving amalgamating finitely many conditions into a given condition at each step of the fusion. Unlike for Laver or Mathias forcing, where only "pure extensions" can be amalgamated, posets with diagonal fusion allow for arbitrary extensions to be amalgamated. This is what is expressed by condition (5). Of course, Laver and Mathias forcing make the ground model meager, and hence do not preserve any MAD families in $\omega^{\omega}$.

REMARK 41. We point out here that all our proofs will go through under the following slight weakening of condition (5) above:

$\left(5^{\prime}\right)$ for any $i \in\left[i_{n}, i_{n+1}\right)$, if $r_{i} \leq p_{i}$, then there is a $q \leq_{n} p$ such that $q_{i} \leq r_{i}$ and $\forall i_{n} \leq i^{\prime}<i\left[q_{i^{\prime}} \leq p_{i^{\prime}}\right]$.

Intuitively, (5) seems stronger than $\left(5^{\prime}\right)$ because (5) allows us to "amalgamate" the $r_{i}$ into $q$ simultaneously, whereas with $\left(5^{\prime}\right)$, we must do this successively, one $i$ at a time. We do not use $\left(5^{\prime}\right)$ in our proofs because it makes the notation more cumbersome, and it does not introduce any new ideas into the proofs. We will leave it to the reader to verify that $\left(5^{\prime}\right)$ is indeed enough for the proofs in this section.

An example of a forcing which, with its usual notion of fusion, satisfies condition $\left(5^{\prime}\right)$ but not condition (5) is Silver forcing.

Before showing that posets with diagonal fusion strongly preserve strongly MAD families, we show that Miller and Sacks forcings have diagonal fusion. We check the details only for Miller forcing, as it is the more difficult case. The proof for the other case is very similar, but easier. 
Definition 42. Let $T$ be a subtree of either $\omega^{<\omega}$ or $2^{<\omega}$. If $s \in T$, we write $\operatorname{succ}_{T}(s)$ to denote $\{s \frown\langle n\rangle: s \frown\langle n\rangle \in T\}$. If $T \subset 2^{<\omega}$, then $\operatorname{split}(T)=$ $\left\{s \in T:\left|\operatorname{succ}_{T}(s)\right|=2\right\}$, while if $T \subset \omega^{<\omega}$, then $\operatorname{split}(T)=\{s \in T$ : $\left.\left|\operatorname{succ}_{T}(s)\right|=\omega\right\}$. In both cases, $\operatorname{split}^{+}(T)=\bigcup\left\{\operatorname{succ}_{T}(s): s \in \operatorname{split}(T)\right\}$. If $n \in \omega, \operatorname{split}_{n}(T)=\{s \in \operatorname{split}(T):|\{t \subsetneq s: t \in \operatorname{split}(T)\}|=n\}$. Finally, $\operatorname{split}_{n}^{+}(T)$ will denote $\bigcup\left\{\operatorname{succ}_{T}(s): s \in \operatorname{split}_{n}(T)\right\}$.

Definition 43. Let $T$ be a subtree of $2^{<\omega}$. We say that $T$ is perfect if $\forall s \in T \exists t \in \operatorname{split}(T)[s \subset t]$. If $T$ is a subtree of $\omega^{<\omega}$, we say that $T$ is superperfect if for each $s \in T$ either $\left|\operatorname{succ}_{T}(s)\right|=1$ or $\left|\operatorname{succ}_{T}(s)\right|=\omega$ and if in addition to this $\forall s \in T \exists t \in \operatorname{split}(T)[s \subset t]$.

Definition 44. $\mathbb{M}$ will denote Miller forcing, $\mathbb{M}=\left\{T \subset \omega^{<\omega}: T\right.$ is superperfect $\}$, ordered by inclusion. $\mathbb{S}$ will denote Sacks forcing, $\mathbb{S}=\{T \subset$ $2^{<\omega}: T$ is perfect\}, ordered by inclusion.

Several distinct notions of fusion can be defined on $\mathbb{M}$. The strongest such notion requires a $\leq_{n}$ extension to preserve all $n$th split nodes. However, this is too strong for proving that $\mathbb{M}$ strongly preserves strongly MAD families because in order to ensure that our extensions stay within the elementary submodel $M$, we need to be able to get away with preserving only finitely many nodes at a time. So we will use the weaker notion of fusion which is sometimes known as diagonal fusion across the Miller tree $T$ (hence the terminology of Definition 40).

Definition 45. Let $T \in \mathbb{M}$. As $T$ is superperfect, there is a natural bijection from $\omega^{<\omega}$ onto $\operatorname{split}(T)$. If $s \in \omega^{<\omega}$, we let $T(s)$ denote the split node of $T$ corresponding to $s$ under this bijection. If $i \in \omega$, then $T(s, i)$ will denote the $i$ th element of $\operatorname{succ}_{T}(T(s))$ under the natural ordering on $\operatorname{succ}_{T}(T(s))$. Finally, if $s \in T$, we write $T_{s}$ to denote $\{t \in T: s \subset t \vee t \subset s\}$.

Given $T \in \mathbb{M}, I_{T}$ as a set will just be $\left\{T_{t}: t \in \operatorname{split}^{+}(T)\right\}$. But to ensure that the conditions of Definition 40 are satisfied we must enumerate this set in a very particular way.

Definition 46. We define a sequence of finite subsets of $\omega^{<\omega}$ as follows. $\Sigma_{0}=\{\langle\rangle\}$. Given $\Sigma_{n}, \Sigma_{n+1}=\left\{s \frown\langle i\rangle: s \in \Sigma_{n} \wedge i \leq n\right\} \cup\{\langle\rangle\}$. Notice that $\Sigma_{n+1} \supset \Sigma_{n}$ and that $\bigcup \Sigma_{n}=\omega^{<\omega}$.

Definition 47. Let $T^{1} \leq T^{0} \in \mathbb{M}$. For any $n \in \omega$, we will say $T^{1} \leq_{n} T^{0}$ if $\forall s \in \Sigma_{n}\left[T^{1}(s)=T^{0}(s)\right]$.

TheOrem 48. M has diagonal fusion.

Proof. We will show that $\left\langle\leq_{n}: n \in \omega\right\rangle$ as defined above witnesses that $\mathbb{M}$ has diagonal fusion. Indeed, it is clear that $\mathbb{M}$ has fusion with respect to $\left\langle\leq_{n}: n \in \omega\right\rangle$. We will check that the other conditions hold. Fix $T \in \mathbb{M}$. We will define $I_{T}$ as follows. Set $i_{n}=\left|\Sigma_{n}\right|-1$. Now, let $e: \omega \rightarrow\left(\omega^{<\omega} \backslash\{\langle\rangle\}\right)$ be a 
one-to-one onto enumeration such that $e^{\prime \prime}\left[0, i_{n}\right)=\Sigma_{n} \backslash\{\langle\rangle\}$. If $i \in\left[i_{n}, i_{n+1}\right)$, then $e(i) \in \Sigma_{n+1} \backslash \Sigma_{n}$. So there is a unique $s \in \Sigma_{n}$ and a unique $j \leq n$ such that $e(i)=s \frown\langle j\rangle$. Let $t_{i}=T(s, j)$. We will set $T_{i}=T_{t_{i}}$. Now, it is clear that $I_{T}=\left\langle T_{i}: i \in \omega\right\rangle=\left\{T_{t}: t \in \operatorname{split}^{+}(T)\right\}$. Therefore, $T_{i} \leq T$, and if $T^{\prime} \leq T$, then $\exists^{\infty} i \in \omega\left[T^{\prime} \not \perp T_{i}\right]$.

Now suppose that $T^{1} \leq_{n} T^{0}$ and let $i<i_{n}$. We must argue that $T_{i}^{1} \leq T_{i}^{0}$. Indeed, if $n=0$, there is nothing to be proved. So suppose that $n>0$. As $e(i) \in \Sigma_{n} \backslash\{\langle\rangle\}$, we can find (unique) $s \in \Sigma_{n-1}$ and $j \leq n-1$ so that $e(i)=s \frown\langle j\rangle$. Notice that $T_{i}^{1}=T_{t_{i}^{1}}^{1}$, where $t_{i}^{1}=T^{1}(s, j)$ and that $T_{i}^{0}=T_{t_{i}^{0}}^{0}$, where $t_{i}^{0}=T^{0}(s, j)$. Since $T^{1} \leq_{n} T^{0}$, we know that $T^{1}\left(s^{\frown}\langle j\rangle\right)=T^{0}\left(s^{\frown}\langle j\rangle\right)$. It follows that $t_{i}^{1}=t_{i}^{0}$. But since $T^{1} \subset T^{0}$, it is easy to see that $T_{t_{i}^{1}}^{1}=T_{t_{i}^{0}}^{1}$ $\subset T_{t_{i}^{0}}^{0}$, whence $T_{i}^{1} \leq T_{i}^{0}$.

Now it only remains to verify clause (5) of Definition 40 . To this end, fix $n \in \omega$ and $T^{0} \in \mathbb{M}$. Let $\left\langle T_{i}^{\prime}: i \in\left[i_{n}, i_{n+1}\right)\right\rangle$ be a sequence such that $\forall i \in\left[i_{n}, i_{n+1}\right)\left[T_{i}^{\prime} \leq T_{i}^{0}\right]$. We wish to amalgamate the $T_{i}^{\prime}$ into $T_{0}$. It is clear that any two distinct $s \neq t \in \Sigma_{n+1} \backslash \Sigma_{n}$ are incomparable nodes in $\omega^{<\omega}$. Therefore, if $s=\tilde{s} \frown\langle j\rangle$ and if $t=\tilde{t} \frown\langle k\rangle$, then $T^{0}(\tilde{s}, j)$ and $T^{0}(\tilde{t}, k)$ are incomparable nodes in the tree $T^{0}$. Thus it follows that if $i \neq i^{\prime}$ are distinct elements in $\left[i_{n}, i_{n+1}\right)$, then $t_{i}^{0}$ and $t_{i^{\prime}}^{0}$ are incomparable nodes in $T^{0}$. But now, we can get $T^{1} \leq_{n} T^{0}$ simply by replacing $T_{t_{i}^{0}}^{0}$ in $T^{0}$ with $T_{i}^{\prime}$ for each $i \in\left[i_{n}, i_{n+1}\right)$. Now, $T^{1}$ is as required, and this finishes the proof.

We now show that if $\mathbb{P}$ has diagonal fusion, then $\mathbb{P}$ strongly preserves all strongly MAD families. The steps are analogous to the steps for Cohen forcing (Theorem 36). We first show that if $\mathbb{P}$ has diagonal fusion, then it is Axiom A.

Lemma 49. If $\mathbb{P}$ has diagonal fusion, then $\mathbb{P}$ is Axiom $A$.

Proof. By assumption, there is a sequence $\left\langle\leq_{n}: n \in \omega\right\rangle$ of partial orderings on $\mathbb{P}$ witnessing that $\mathbb{P}$ has fusion. We will check that this same sequence also witnesses that $\mathbb{P}$ is Axiom $A$. To this end, suppose that $\stackrel{x}{ }$ is a $\mathbb{P}$ name such that $\Vdash \stackrel{\circ}{x} \in \mathbf{V}$. Let $p$ be any condition and let $n \in \omega$. We build a sequence $\left\langle p^{m}: m \in \omega\right\rangle$ with $p^{0}=p$ such that $p^{m+1} \leq_{n+m} p^{m}$ as follows. Suppose that at stage $m+1$ we are given $p^{m}$. Let $I_{p^{m}}=\left\langle p_{i}^{m}: i \in \omega\right\rangle$. For each $i \in$ $\left[i_{n+m}, i_{n+m+1}\right)$ choose $r_{i}^{m} \leq p_{i}^{m}$ and $x_{i}^{m}$ such that $r_{i}^{m} \Vdash \stackrel{\circ}{x}=x_{i}^{m}$. Now, we can find a condition $p^{m+1} \leq_{n+m} p^{m}$ such that $\forall i \in\left[i_{n+m}, i_{n+m+1}\right)\left[p_{i}^{m+1} \leq r_{i}^{m}\right]$, where $I_{p^{m+1}}=\left\langle p_{i}^{m+1}: i \in \omega\right\rangle$. Let $X=\left\{x_{i}^{m}: m \in \omega \wedge i \in\left[i_{n+m}, i_{n+m+1}\right)\right\}$ and let $q \in \mathbb{P}$ be such that $\forall m \in \omega\left[q \leq_{n+m} p^{m}\right]$. $X$ is clearly a countable set. We will argue that $q \Vdash \stackrel{\circ}{x} \in X$. Put $I_{q}=\left\langle q_{i}: i \in \omega\right\rangle$. Let $r \leq q$. Find $m$ and $i \in\left[i_{n+m}, i_{n+m+1}\right)$ such that $r \not \perp q_{i}$. Since $q \leq_{n+m+1} p^{m+1}$, we have $q_{i} \leq p_{i}^{m+1} \leq r_{i}^{m}$. But then we can choose $s \in \mathbb{P}$ extending both $r$ and $r_{i}^{m}$, whence $s \Vdash \stackrel{\circ}{x}=x_{i}^{m} \in X$. 
Lemma 50. Let $\mathbb{P}$ be a poset with diagonal fusion. Let $f$ be a $\mathbb{P}$ name so that $\Vdash f$ is an infinite partial function. Let $\left\{f_{l}: l \in \omega\right\}$ be a set of $\mathbb{P}$ names such that $\forall l \in \omega\left[\Vdash f_{l} \subset \stackrel{\circ}{f}\right.$ is an infinite partial function $]$. Let $p \in \mathbb{P}$ and let $n \in \omega$. There is $q \leq_{n} p$ and an infinite partial function $g$ such that:

(1) $\forall l \in \omega\left[q \Vdash\left|g \cap \stackrel{\circ}{f}_{l}\right|=\omega\right]$.

(2) $\forall k \in \operatorname{dom}(g) \exists i\left[q_{i} \Vdash k \in \operatorname{dom}(f) \wedge \stackrel{\circ}{f}(k)=g(k)\right]$, where $I_{q}=\left\langle q_{i}\right.$ : $i \in \omega\rangle$.

Proof. We will build $g$ by induction as the union of an increasing sequence of finite partial functions $g_{j}$. In fact, we will build two sequences $\left\langle g_{j}:-1 \leq\right.$ $j<\omega\rangle$ and $\left\langle p^{j}: j \in \omega\right\rangle \subset \mathbb{P}$ such that:

(a) $p^{0}=p$ and $p^{j+1} \leq_{n+j} p^{j}$.

(b) $g_{-1}=0$ and $g_{j-1} \subset g_{j}$ is a finite partial function.

(c) For each $i \in\left[i_{n+j}, i_{n+j+1}\right)$ and for each $l \leq j$ there is a $k(i, j, l)>j$ and an $m(i, j, l) \in \omega$ such that $\left[p_{i}^{j+1} \Vdash k(i, j, l) \in \operatorname{dom}\left(f_{l}^{i}\right) \wedge \stackrel{\circ}{l}_{l}(k(i, j, l))\right.$ $=m(i, j, l)]$, where $I_{p^{j+1}}=\left\langle p_{i}^{j+1}: i \in \omega\right\rangle$.

(d) $g_{j}=g_{j-1} \cup\left\{\langle k(i, j, l), m(i, j, l)\rangle: i \in\left[i_{n+j}, i_{n+j+1}\right) \wedge l \leq j\right\}$.

Let $q \in \mathbb{P}$ be such that $q \leq_{n+j} p^{j}$ and let $g=\bigcup g_{j}$. We will first argue that $q$ and $g$ satisfy clauses (1) and (2). Put $I_{q}=\left\langle q_{i}: i \in \omega\right\rangle$. To verify (1), fix $l \in \omega$. Let $r \leq q$ and $k \in \omega$ be given. We know $\exists^{\infty} i \in \omega\left[r \not \perp q_{i}\right]$. Choose $j \geq k, l$ and $i \in\left[i_{n+j}, i_{n+j+1}\right)$ such that $r \not \perp q_{i}$. As $l \leq j$, there is a $k(i, j, l)>j \geq k$ and an $m(i, j, l) \in \omega$ such that $k(i, j, l) \in \operatorname{dom}(g), g(k(i, j, l))=m(i, j, l)$, and $p_{i}^{j+1} \Vdash k(i, j, l) \in \operatorname{dom}\left(\stackrel{\circ}{f}_{l}\right) \wedge \stackrel{\circ}{f}_{l}(k(i, j, l))=m(i, j, l)$. But $q \leq_{n+j+1} p^{j+1}$. Therefore, $q_{i} \leq p_{i}^{j+1}$. Hence, we can find an $s \in \mathbb{P}$ extending both $r$ and $p_{i}^{j+1}$, whence $s \Vdash k(i, j, l) \in \operatorname{dom}\left(\AA_{l}^{\prime}\right) \wedge \stackrel{\circ}{f}_{l}(k(i, j, l))=m(i, j, l)=g(k(i, j, l))$. As $k(i, j, l)>k$, this verifies (1).

Next, to verify (2), suppose that $k \in \operatorname{dom}(g)$. By clause (d), $k=k(i, j, l)$ and $g(k)=m(i, j, l)$ for some $l \leq j$ and $i \in\left[i_{n+j}, i_{n+j+1}\right)$. Clause (c) implies that $p_{i}^{j+1} \Vdash k \in \operatorname{dom}\left(\AA_{l}^{\prime}\right) \wedge \stackrel{\circ}{f}_{l}(k)=m(i, j, l)=g(k)$. But since $\stackrel{\circ}{l}_{l}$ is forced to be a subfunction of $\stackrel{\circ}{f}, p_{i}^{j+1} \Vdash k \in \operatorname{dom}(f) \wedge \stackrel{\circ}{f}(k)=g(k)$. As $q \leq_{n+j+1} p^{j+1}$, $q_{i} \leq p_{i}^{j+1}$. Therefore, $q_{i} \Vdash k \in \operatorname{dom}(\stackrel{\circ}{)} \wedge \stackrel{\circ}{f}(k)=g(k)$, which is as required.

Now, we describe the construction of $\left\langle g_{j}:-1 \leq j<\omega\right\rangle$ and $\left\langle p^{j}: j \in \omega\right\rangle$. We set $g_{-1}=0$ and $p^{0}=p$. At stage $j \geq 0$ suppose we are given $g_{j-1}$ and $p^{j}$. Put $I_{p^{j}}=\left\langle p_{i}^{j}: i \in \omega\right\rangle$. Since $g_{j-1}$ is a finite partial function, we can set $k=\max \left(\operatorname{dom}\left(g_{j-1}\right)\right)$. As each $f_{l}$ is forced to be an infinite partial function, we can find a sequence of conditions $\left\langle r_{i}^{j}: i \in\left[i_{n+j}, i_{n+j+1}\right)\right\rangle$ and two sequences of numbers $\left\langle k(i, j, l): i \in\left[i_{n+j}, i_{n+j+1}\right) \wedge l \leq j\right\rangle$ and $\langle m(i, j, l)$ : $\left.i \in\left[i_{n+j}, i_{n+j+1}\right) \wedge l \leq j\right\rangle$ satisfying:

(i) $r_{i}^{j} \leq p_{i}^{j}$ and $r_{i}^{j} \Vdash k(i, j, l) \in \operatorname{dom}\left(\stackrel{\circ}{l}_{l}\right) \wedge \stackrel{\circ}{f}_{l}(k(i, j, l))=m(i, j, l)$. 
(ii) $k(i, j, l)>\max (\{k, j\})$ and $k(i, j, l)<k\left(i^{\prime}, j, l^{\prime}\right)$ whenever $(i, l)<$ $\left(i^{\prime}, l^{\prime}\right)$ lexicographically.

By clause (5) of Definition 40, we can find $p^{j+1} \leq_{n+j} p^{j}$ such that for each $i \in\left[i_{n+j}, i_{n+j+1}\right), p_{i}^{j+1} \leq r_{i}^{j}$. Since the $k(i, j, l)$ are distinct for distinct pairs $(i, l)$, we can set $g_{j}=g_{j-1} \cup\left\{\langle k(i, j, l), m(i, j, l)\rangle: i \in\left[i_{n+j}, i_{n+j+l}\right) \wedge l \leq j\right\}$. It is clear that $g_{j}$ and $p^{j+1}$ are as required.

Lemma 51. Let $\mathscr{A} \subset \omega^{\omega}$ be a strongly $M A D$ family and let $\mathbb{P}$ be a poset with diagonal fusion. Let $M \prec H(\theta)$ be countable with $\mathscr{A}, \mathbb{P} \in M$. Suppose $\stackrel{\circ}{f} M$ is a $\mathbb{P}$ name such that $\Vdash \stackrel{\circ}{f}$ is an infinite partial function avoiding $\mathscr{A}$. Suppose $h \in \mathscr{A}$ covers $M$ with respect to $\mathscr{A}$. Let $p \in M$ be a condition and let $j \in \omega$. There is a $r \leq p$ such that $r \in M$ and $\exists k>j[r \Vdash k \in \operatorname{dom}(f) \wedge \stackrel{\circ}{f}(k)$ $=h(k)$.

Proof. We can apply Lemma 34 to $\stackrel{\circ}{f}$ to find a set of names $\left\{\AA_{l}: l \in \omega\right\}$ $\in M$ that satisfy clauses (1) and (2) of Lemma 34. Notice that the hypotheses of Lemma 50 are satisfied by $\mathbb{P}, \stackrel{\circ}{f},\left\{\stackrel{\circ}{f}_{l}: l \in \omega\right\}$, and $p$. So we can find $q \leq p$ with $q \in M$ and an infinite partial function $g \in M$ which satisfy clauses (1) and (2) of Lemma 50. Put $I_{q}=\left\langle q_{i}: i \in \omega\right\rangle$. Notice that since $q \in M, I_{q} \in M$, and by elementarity, $I_{q} \subset M$. Now, observe that clause (2) of Lemma 34 and clause (1) of Lemma 50 together imply that $g$ avoids $\mathscr{A}$. Therefore, $|h \cap g|$ $=\omega$. Choose $k>j$ such that $k \in \operatorname{dom}(g)$ and $h(k)=g(k)$. By clause (2) of Lemma 50, there is $i \in \omega$ such that $q_{i} \Vdash k \in \operatorname{dom}(\stackrel{\circ}{f}) \wedge f(k)=g(k)$ $=h(k)$. As $q_{i} \in M$ and $q_{i} \leq q$, we can set $r=q_{i}$. Clearly, $r$ and $k$ are as required.

Theorem 52. Let $\mathscr{A} \subset \omega^{\omega}$ be a strongly MAD family. Let $\mathbb{P}$ be a poset with diagonal fusion. Then $\mathbb{P}$ strongly preserves $\mathscr{A}$.

Proof. Fix a countable elementary submodel $M \prec H(\theta)$ with $\mathscr{A}, \mathbb{P} \in M$. Let $h \in \mathscr{A}$ cover $M$ with respect to $\mathscr{A}$ and let $p \in M$ be a condition. We must find a $q \leq p$ which is $(M, \mathbb{P})$ generic such that $q \Vdash h$ covers $M[\stackrel{\circ}{G}]$ with respect to $\mathscr{A}$. We will use Lemma 49 to ensure that $q$ is $(M, \mathbb{P})$ generic and use Lemma 51 to ensure that $q \Vdash h$ covers $M[\stackrel{\circ}{G}]$ with respect to $\mathscr{A}$. Let $\left\langle\stackrel{\circ}{\alpha}_{n}: n \in \omega\right\rangle$ enumerate all $\stackrel{\circ}{\alpha} \in M \cap \mathbf{V}^{\mathbb{P}}$ such that $\Vdash \stackrel{\circ}{\alpha}$ is an ordinal. Let $\left\langle\dot{f}_{j}: j \in \omega\right\rangle$ enumerate all $f \in M \cap \mathbf{V}^{\mathbb{P}}$ such that $\Vdash f$ is an infinite partial function avoiding $\mathscr{A}$. We will build a sequence $\left\langle p^{n}: n \in \omega\right\rangle \subset \mathbb{P} \cap M$ such that the following hold:

(a) $p^{0}=p$ and $p^{n+1} \leq_{n} p^{n}$.

(b) $p^{n+1} \Vdash \stackrel{\circ}{\alpha}_{n} \in M$.

(c) For each $i \in\left[i_{n}, i_{n+1}\right)$ and for each $j \leq n$ there is a $k(i, n, j)>n$ such that $p_{i}^{n+1} \Vdash k(i, n, j) \in \operatorname{dom}\left(\stackrel{\circ}{f}_{j}\right) \wedge \stackrel{\circ}{f}_{j}(k(i, n, j))=h(k(i, n, j))$. 
Let $q \in \mathbb{P}$ be a condition such that $q \leq_{n} p^{n}$ for all $n \in \omega$. We will first argue that $q$ is as required. Indeed, it is clear from (b) above that $q$ is $(M, \mathbb{P})$ generic. We will argue that $q \Vdash h$ covers $M[\stackrel{\circ}{G}]$ with respect to $\mathscr{A}$. Let $G$ be a $(\mathbf{V}, \mathbb{P})$ generic filter with $q \in G$ and let $f \in M[G]$ be an infinite partial function avoiding $\mathscr{A}$. By elementarity of $M$, there is $f \in M$ with $f[G]=f$ such that $\Vdash f$ is an infinite partial function avoiding $\mathscr{A}$. Therefore, $\stackrel{\circ}{f}=\dot{f}_{j}$ for some $j \in \omega$. It is enough to show that $q \Vdash\left|h \cap \stackrel{\circ}{f}_{j}\right|=\omega$. Fix $r \leq q$ and $k \in \omega$. Put $I_{q}=\left\{q_{i}: i \in \omega\right\}$. We know that $\exists^{\infty} i \in \omega\left[\begin{array}{lll}r & \not \perp & q_{i}\end{array}\right]$. So we can choose $n \geq \max \{j, k\}$ and $i \in\left[i_{n}, i_{n+1}\right)$ such that $r \not \perp q_{i}$. Since $j \leq n$, by (c) above, there is $k(i, n, j)>$ $n$ such that $p_{i}^{n+1} \Vdash k(i, n, j) \in \operatorname{dom}\left(\dot{\circ}_{j}\right) \wedge \dot{f}_{j}(k(i, n, j))=h(k(i, n, j))$. But since $q \leq{ }_{n+1} p^{n+1}, q_{i} \leq p_{i}^{n+1}$. So we may choose $s \leq r$ with the property that $s \Vdash k(i, n, j) \in \operatorname{dom}\left(\stackrel{\circ}{f}_{j}\right) \wedge \dot{\circ}_{j}(k(i, n, j))=h(k(i, n, j))$. This is as required because $k(i, n, j)>k$.

We now describe how to construct $\left\langle p^{n}: n \in \omega\right\rangle$. Set $p^{0}=p$ and suppose that at stage $n, p^{n} \in M$ is given to us. We first apply Lemma 49 to $p^{n}$ and $\stackrel{\circ}{\alpha}_{n}$ within $M$ to find $\tilde{p}^{n} \leq_{n} p^{n}$ with $\tilde{p}^{n} \in M$ such that $\tilde{p}^{n} \Vdash$ $\stackrel{\circ}{\alpha}_{n} \in M$. Put $I_{\tilde{p}^{n}}=\left\langle\tilde{p}_{i}^{n}: i \in \omega\right\rangle$. Note that $I_{\tilde{p}^{n}} \subset M$. Fix any $i \in$ $\left[i_{n}, i_{n+1}\right)$. Notice that $\tilde{p}_{i}^{n} \in M$. As $\left\langle\dot{f}_{j}: j \leq n\right\rangle \subset M$, we can apply Lemma 51 to $\tilde{p}_{i}^{n} n+1$ times to find $r_{i}^{n} \leq \tilde{p}_{i}^{n}$ with $r_{i}^{n} \in M$ and numbers $k(i, n, 0), \ldots, k(i, n, n) \in \omega$, all of them greater than $n$, such that $\forall j \leq n\left[r_{i}^{n} \Vdash k(i, n, j) \in \operatorname{dom}\left(\dot{f}_{j}\right) \wedge \dot{f}_{j}(k(i, n, j))=h(k(i, n, j))\right]$. Now, $\left\langle r_{i}^{n}\right.$ : $\left.i \in\left[i_{n}, i_{n+1}\right)\right\rangle$ is a finite sequence of things in $M$. Therefore $\left\langle r_{i}^{n}: i \in\right.$ $\left.\left[i_{n}, i_{n+1}\right)\right\rangle \in M$. Hence, we can apply (5) of Definition 40 to $\tilde{p}^{n}$ to find $p^{n+1} \leq_{n} \tilde{p}^{n}$ with $p^{n+1} \in M$ such that $\forall i \in\left[i_{n}, i_{n+1}\right)\left[p_{i}^{n+1} \leq r_{i}^{n}\right]$. It is clear that $p^{n+1}$ is as needed.

Putting Theorems 48 and 52 together, we get

Corollary 53. If $\mathscr{A} \subset \omega^{\omega}$ is a strongly $M A D$ family, then $\mathbb{M}$ and $\mathbb{S}$ strongly preserve $\mathscr{A}$.

\section{Some preservation theorems for countable support iterations.} Our main goal in this section is to prove that the property of strongly preserving a strongly MAD family is preserved by the countable support (CS) iteration of proper posets. By the results of the last section, this will imply that the countable support iterations of Sacks and Miller forcings strongly preserve strongly MAD families.

We assume that the reader is familiar with the basic theory of iterated forcing, including some preservation theorems, such as the preservation of properness. The reader may consult [1], [7], [22] or [23] for a good introduction. Our presentation will generally follow that of Abraham [1]. 
En route to proving our main theorem, we answer a question of Kellner and Shelah [16] by proving the following. Suppose $\gamma$ is a limit ordinal and that $\left\langle\mathbb{P}_{\alpha}, \mathbb{Q}_{\alpha}: \alpha \leq \gamma\right\rangle$ is a countable support iteration of proper posets. If for all $\alpha<\gamma, \mathbb{P}_{\alpha}$ does not add an eventually different real, then $\mathbb{P}_{\gamma}$ does not add an eventually different real either. Since not adding an eventually different real is known to be equivalent to not making $\mathbf{V} \cap \omega^{\omega}$ meager (see [3]), our result shows that a CS iteration of proper posets of limit length does not make $\mathbf{V} \cap \omega^{\omega}$ meager if no initial segment does. A partial result towards our theorem was obtained by Kellner and Shelah [16], who proved it for the case of Suslin forcings as well as some nep forcings. Shelah, Judah and Goldstern (see [22]) have shown that the countable support iteration of posets which preserve $\Xi_{\mathbf{C}}$ itself preserves $\Xi_{\mathbf{C}}$ (Definition 27 ). The property of preserving $\sqsubseteq_{\mathbf{C}}$ is stronger than that of not adding eventually different reals (though they are known to be equivalent for Suslin proper posets). On the other hand, our result puts a condition on initial segments of the iteration, and not on the iterands.

Before giving the proofs of our results, we collect together some basic facts about countable support iterations which we will use.

Lemma 54 (see the proof of Lemma 2.8 in [1]). Let $\gamma$ be a limit ordinal and let $\left\langle\mathbb{P}_{\alpha}, \mathbb{Q}_{\alpha}: \alpha \leq \gamma\right\rangle$ be a CS iteration. Let $M \prec H(\theta)$ be countable and suppose that $\left\langle\mathbb{P}_{\alpha}, \mathbb{Q}_{\alpha}: \alpha \leq \gamma\right\rangle \in M$. Put $\gamma^{\prime}=\sup (\gamma \cap M)$ and let $\left\langle\gamma_{n}: n \in \omega\right\rangle \subset \gamma \cap M$ be an increasing sequence that is cofinal in $\gamma^{\prime}$. Suppose that $\left\langle q_{n}: n \in \omega\right\rangle$ and $\left\langle\dot{p}_{n}: n \in \omega\right\rangle$ are two sequences such that the following hold:

(1) $q_{n} \in \mathbb{P}_{\gamma_{n}}$ and $q_{n+1}\left\lceil\gamma_{n}=q_{n}\right.$.

(2) $\stackrel{\circ}{p}_{n} \in \mathbf{V}^{\mathbb{P}_{\gamma_{n}}}$ and $q_{n} \Vdash \vdash_{n} \stackrel{\circ}{p}_{n} \in M \cap \mathbb{P}_{\gamma} \wedge \stackrel{\circ}{p}_{n}\left\lceil\gamma_{n} \in \stackrel{\circ}{G}_{\gamma_{n}}\right.$.

(3) $q_{n+1} \Vdash_{\gamma_{n+1}} \stackrel{\circ}{p+1}_{n+\grave{p}_{n}}$.

If $q=\left(\bigcup q_{n}\right) \frown \grave{\mathbb{1}} \in \mathbb{P}_{\gamma}$, then $\forall n \in \omega\left[q \Vdash_{\gamma} \stackrel{\circ}{n}_{n} \in \stackrel{\circ}{\gamma}_{\gamma}\right]$.

Lemma 55 (see Lemma 2.8 of [1]). Let $\left\langle\mathbb{P}_{\alpha}, \AA_{\alpha}: \alpha \leq \gamma\right\rangle$ be a CS iteration such that $\forall \alpha<\gamma\left[\Vdash_{\alpha} \mathbb{Q}_{\alpha}\right.$ is proper $]$. Let $M \prec H(\theta)$ be countable and suppose that $\left\langle\mathbb{P}_{\alpha}, \mathbb{Q}_{\alpha}: \alpha \leq \gamma\right\rangle \in M$. Let $\gamma_{0} \in \gamma \cap M$ and suppose that $q_{0} \in \mathbb{P}_{\gamma_{0}}$ is an $\left(M, \mathbb{P}_{\gamma_{0}}\right)$ generic condition. Suppose $\stackrel{\circ}{0}_{0} \in \mathbf{V}^{\mathbb{P}_{\gamma_{0}}}$ and

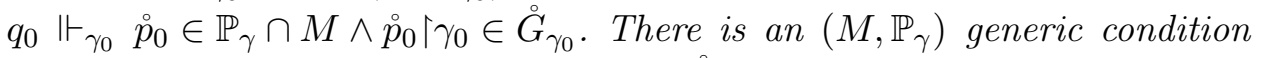
$q \in \mathbb{P}_{\gamma}$ such that $q\left\lceil\gamma_{0}=q_{0}\right.$ and $q \Vdash_{\gamma} \stackrel{\circ}{0}_{0} \in \stackrel{\circ}{G}_{\gamma}$.

Definition 56. Let $\mathbb{P}$ and $\mathbb{Q}$ be posets and suppose that $\pi: \mathbb{Q} \rightarrow \mathbb{P}$ is an onto map. We say that $\pi$ is a projection if the following hold:

(1) $\pi$ is order preserving. That is, if $q_{1} \leq q_{0}$, then $\pi\left(q_{1}\right) \leq \pi\left(q_{0}\right)$.

(2) For every $q_{0} \in \mathbb{Q}$ if $p \leq \pi\left(q_{0}\right)$, then $\exists q_{1} \leq q_{0}\left[\pi\left(q_{1}\right)=p\right]$. 
Definition 57. Let $\left(\mathbb{P}, \leq_{\mathbb{P}}\right)$ and $\left(\mathbb{Q}, \leq_{\mathbb{Q}}\right)$ be posets and let $\pi: \mathbb{Q} \rightarrow \mathbb{P}$ be a projection. If $G \subset \mathbb{P}$ is a $(\mathbf{V}, \mathbb{P})$ generic filter, then in $\mathbf{V}[G]$ we define the poset $\mathbb{Q} / G=\{q \in \mathbb{Q}: \pi(q) \in G\}$ ordered by $\leq_{\mathbb{Q}}$. In $\mathbf{V}$, we let $\mathbb{Q} / G$ be a full $\mathbb{P}$ name for $\mathbb{Q} / G$.

Lemma 58 (see Section 4 of [7]). Let $\mathbb{P}$ and $\mathbb{Q}$ be posets and let $\pi: \mathbb{Q} \rightarrow \mathbb{P}$ be a projection. There is a dense embedding $i: \mathbb{Q} \rightarrow \mathbb{P} * \mathbb{Q} /{ }^{\circ}$ given by $i(q)=\langle\pi(q), q\rangle$. Moreover, if $\left\langle\mathbb{P}_{\alpha}, \mathbb{Q}_{\alpha}: \alpha \leq \gamma\right\rangle$ is an iteration, then for each $\alpha \leq \gamma$ the map $\pi_{\gamma \alpha}: \mathbb{P}_{\gamma} \rightarrow \mathbb{P}_{\alpha}$ given by $\pi_{\gamma \alpha}(p)=p\lceil\alpha$ is a projection. Therefore, if $\alpha \leq \gamma$ and if $G_{\gamma} \subset \mathbb{P}_{\gamma}$ is a $\left(\mathbf{V}, \mathbb{P}_{\gamma}\right)$ generic filter, then there is $a\left(\mathbf{V}\left[G_{\alpha}\right], \mathbb{P}_{\gamma} / G_{\alpha}\right)$ generic filter $H$ such that in $\mathbf{V}\left[G_{\gamma}\right], G_{\gamma}=G_{\alpha} * H$ holds. In fact, this $H$ is equal to $G_{\gamma}$.

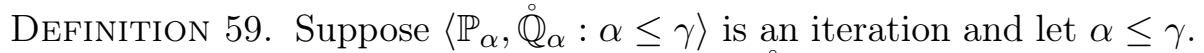
By Lemma 58, $\mathbb{P}_{\gamma}$ densely embeds into $\mathbb{P}_{\alpha} * \mathbb{P}_{\gamma} / \stackrel{G}{G}_{\alpha}$. Thus we may think of

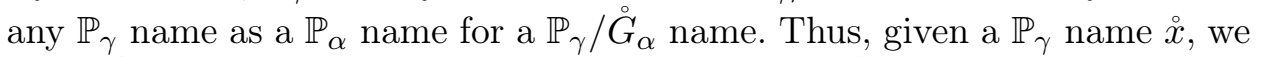
use $\stackrel{x}{x}\left[\stackrel{\circ}{G}_{\alpha}\right]$ to denote a canonical $\mathbb{P}_{\alpha}$ name for a $\mathbb{P}_{\gamma} / \stackrel{\circ}{G}_{\alpha}$ name representing $\stackrel{\circ}{x}$. If $G_{\alpha}$ is a $\left(\mathbf{V}, \mathbb{P}_{\alpha}\right)$ generic filter, we will write $\stackrel{\circ}{x}\left[G_{\alpha}\right]$ to denote the evaluation of $\stackrel{x}{[}\left[G_{\alpha}\right]$ by $G_{\alpha}$. Therefore, if $G_{\gamma}$ is a $\left(\mathbf{V}, \mathbb{P}_{\gamma}\right)$ generic filter, then in $\mathbf{V}\left[G_{\gamma}\right]$, $\stackrel{\circ}{x}\left[G_{\gamma}\right]=\stackrel{\circ}{x}\left[G_{\alpha}\right][H]$ holds, where $H$ is a $\left(\mathbf{V}\left[G_{\alpha}\right], \mathbb{P}_{\gamma} / G_{\alpha}\right)$ generic filter such that $G_{\gamma}=G_{\alpha} * H$. By Lemma $58, H=G_{\gamma}$.

We are now ready to prove our main results. We will begin by showing that a CS iteration of proper forcings of limit length does not add an eventually different real if no initial segment does.

Lemma 60. Let $\mathbf{V}_{0} \subset \mathbf{V}_{1}$ be transitive universes satisfying $Z F C$ and suppose that for every $f \in \omega^{\omega} \cap \mathbf{V}_{1}$ there is a slalom $S \in \mathbf{V}_{0}$ such that $\exists^{\infty} n \in \omega[f(n) \in S(n)]$. No $f \in \omega^{\omega} \cap \mathbf{V}_{1}$ is eventually different from $\omega^{\omega} \cap \mathbf{V}_{0}$.

Proof. Working in $\mathbf{V}_{0}$ partition $\omega$ into a sequence of intervals $\left\langle I_{n}: n \in \omega\right\rangle$ $\in \mathbf{V}_{0}$ such that $\forall n \in \omega\left[\left|I_{n}\right|=2^{n}\right]$. Put $X=\bigcup \omega^{I_{n}}$. Notice that for each $n \in \omega, \omega^{I_{n}} \cap \mathbf{V}_{1}=\omega^{I_{n}} \cap \mathbf{V}_{0}$. Let $f \in \omega^{\omega} \cap \mathbf{V}_{1}$. Working in $\mathbf{V}_{1}$ define a function $F: \omega \rightarrow X$ by stipulating that $F(n)=f\left\lceil I_{n} \in \omega^{I_{n}}\right.$. By hypothesis, we can find $S \in \mathbf{V}_{0}$ such that:

(1) $S: \omega \rightarrow[X]^{<\omega}$.

(2) $\forall n \in \omega\left[|S(n)| \leq 2^{n}\right]$.

(3) $\exists^{\infty} n \in \omega[F(n) \in S(n)]$.

We may assume that for all $n \in \omega, S(n) \subset \omega^{I_{n}}$ and that $|S(n)|=2^{n}$ because we may modify $S$ to make both of these things true without affecting the truth of (3) above. Put $S(n)=\left\{\sigma_{0}^{n}, \ldots, \sigma_{2^{n}-1}^{n}\right\}$ and $I_{n}=\left\{i_{0}^{n}, \ldots, i_{2^{n}-1}^{n}\right\}$. For each $n \in \omega$ and for each $0 \leq j<2^{n}$ define $g\left(i_{j}^{n}\right)=\sigma_{j}^{n}\left(i_{j}^{n}\right)$. This definition makes sense because by assumption, $\sigma_{j}^{n} \in \omega^{I_{n}}$, and so $\sigma_{j}^{n}$ is defined at $i_{j}^{n}$. Clearly, $g \in \omega^{\omega} \cap \mathbf{V}_{0}$. We will argue in $\mathbf{V}_{1}$ that $\exists^{\infty} n \in \omega[f(n)=g(n)]$. 
We know that $A=\{n \in \omega: F(n) \in S(n)\}$ is infinite. For each $n \in A$, there is a $0 \leq j_{n}<2^{n}$ such that $f \backslash I_{n}=\sigma_{j_{n}}^{n}$. Therefore, for each $n \in A$, $g\left(i_{j_{n}}^{n}\right)=\sigma_{j_{n}}^{n}\left(i_{j_{n}}^{n}\right)=f\left(i_{j_{n}}^{n}\right)$. Since the $I_{n}$ are disjoint, the set $\left\{i_{j_{n}}^{n}: n \in A\right\}$ is infinite, and we are done.

Theorem 61. Let $\gamma$ be a limit ordinal and let $\left\langle\mathbb{P}_{\alpha}, \AA_{\alpha}: \alpha \leq \gamma\right\rangle$ be a $C S$ iteration such that $\forall \alpha<\gamma\left[\vdash_{\alpha} \mathbb{Q}_{\alpha}\right.$ is proper $]$. Suppose that for all $\alpha<\gamma, \mathbb{P}_{\alpha}$ does not add an eventually different real. Then $\mathbb{P}_{\gamma}$ does not add an eventually different real either.

Proof. Let $\stackrel{\circ}{f}$ be a $\mathbb{P}_{\gamma}$ name such that $\Vdash_{\gamma} \stackrel{\circ}{f} \in \omega^{\omega}$, and let $p_{0} \in \mathbb{P}_{\gamma}$ be a condition. Fix a countable $M \prec H(\theta)$ with $\left\langle\mathbb{P}_{\alpha}, \mathbb{Q}_{\alpha}: \alpha \leq \gamma\right\rangle, \stackrel{\circ}{f}, p_{0} \in M$. Let $S: \omega \rightarrow[\omega]^{<\omega}$ be a slalom such that for all $f \in \omega^{\omega} \cap M, \forall^{\infty} n \in \omega$ $[f(n) \in S(n)]$. We will find $q \in \mathbb{P}_{\gamma}$ such that $q \Vdash_{\gamma} p_{0} \in G_{\gamma}$ and $q \Vdash_{\gamma} \exists^{\infty} n \in \omega$ $[\stackrel{\circ}{f}(n) \in S(n)]$. By Lemma 60 , this is sufficient. Put $\gamma^{\prime}=\sup (M \cap \gamma)$ and let $\left\langle\gamma_{n}: n \in \omega\right\rangle \subset M \cap \gamma$ be an increasing sequence that is cofinal in $\gamma^{\prime}$. We will build two sequences $\left\langle q_{n}: n \in \omega\right\rangle$ and $\left\langle\dot{p}_{n}: n \in \omega\right\rangle$ such that the following hold:

(1) $q_{n} \in \mathbb{P}_{\gamma_{n}}, q_{n}$ is $\left(M, \mathbb{P}_{\gamma_{n}}\right)$ generic, and $q_{n+1}\left\lceil\gamma_{n}=q_{n}\right.$.

(2) $\stackrel{\circ}{p}_{0}=p_{0}, \stackrel{\circ}{p}_{n} \in \mathbf{V}^{\mathbb{P} \gamma_{n}}$, and $q_{n} \Vdash{ }_{\gamma_{n}} \stackrel{\circ}{p}_{n} \in M \cap \mathbb{P}_{\gamma} \wedge \stackrel{\circ}{p}_{n}\left\lceil\gamma_{n} \in \stackrel{\circ}{G}_{\gamma_{n}}\right.$.

(3) $q_{n+1} \Vdash_{\gamma_{n+1}} \stackrel{\circ}{p}_{n+1} \leq \stackrel{\circ}{n}_{n}$.

(4) $q_{n+1} \Vdash_{\gamma_{n+1}} \stackrel{\circ}{p}_{n+1} \Vdash_{\mathbb{P}_{\gamma} / \stackrel{\circ}{G}_{\gamma_{n+1}}} \exists k \geq n\left[\stackrel{\circ}{\mathrm{f}}\left[{\stackrel{\circ}{\sigma_{\gamma_{n+1}}}}\right](k) \in S(k)\right]$.

Before showing how to build such a sequence, we will argue that it is sufficient

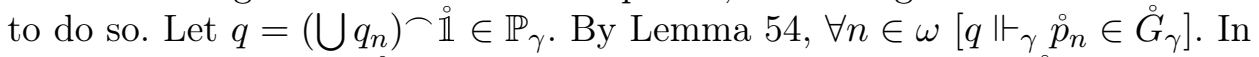
particular, $q \Vdash_{\gamma} p_{0} \in \dot{G}_{\gamma}$. We will argue that $q \Vdash_{\gamma} \exists^{\infty} n \in \omega[\stackrel{\circ}{f}(n) \in S(n)]$. Indeed, let $r \leq q$ and let $n \in \omega$. Fix a $\left(\mathbf{V}, \mathbb{P}_{\gamma}\right)$ generic filter $G_{\gamma}$, with $r \in G_{\gamma}$. By Lemma 58, we know that $G_{\gamma}$ is $\left(\mathbf{V}\left[G_{\gamma_{n+1}}\right], \mathbb{P}_{\gamma} / G_{\gamma_{n+1}}\right)$ generic and that in $\mathbf{V}\left[G_{\gamma}\right], G_{\gamma}=G_{\gamma_{n+1}} * G_{\gamma}$ holds. Notice that $q \in G_{\gamma}$ and therefore $q_{n+1} \in$ $G_{\gamma_{n+1}}$. Also, since $\stackrel{\circ}{n+1}_{n+1}$ is a $\mathbb{P}_{\gamma_{n+1}}$ name, $\stackrel{\circ}{n+1}_{n+1}\left[G_{\gamma}\right]={\stackrel{\circ}{p_{n+1}}}_{n}\left[G_{\gamma_{n+1}}\right]$. It follows from clauses (2) and (4) that $\stackrel{p}{p}_{n+1}\left[G_{\gamma}\right] \in M \cap \mathbb{P}_{\gamma}$, that $\stackrel{\circ}{p}_{n+1}\left[G_{\gamma}\right] \mid \gamma_{n+1} \in$ $G_{\gamma_{n+1}}$, and that in $\mathbf{V}\left[G_{\gamma_{n+1}}\right], \stackrel{\circ}{p}_{n+1}\left[G_{\gamma}\right] \Vdash_{\mathbb{P}_{\gamma} / G_{\gamma_{n+1}}} \exists k \geq n\left[\stackrel{\circ}{f}\left[G_{\gamma_{n+1}}\right](k) \in\right.$ $S(k)]$. However, as observed above, Lemma 54 implies that $\stackrel{\circ}{n+1}_{n}\left[G_{\gamma}\right] \in G_{\gamma}$. Therefore in $\mathbf{V}\left[G_{\gamma}\right]$, there is a $k \geq n$ such that $\stackrel{f}{[}\left[G_{\gamma_{n+1}}\right]\left[G_{\gamma}\right](k) \in S(k)$. But $\stackrel{\circ}{f}\left[G_{\gamma_{n+1}}\right]\left[G_{\gamma}\right]=\stackrel{\circ}{f}\left[G_{\gamma}\right]$. So $\stackrel{\circ}{f}\left[G_{\gamma}\right](k) \in S(k)$. Since $r \in G_{\gamma}$, we may find $s \leq r$ such that $s \Vdash_{\gamma} f(k) \in S(k)$. As $k \geq n$, this finishes the proof.

We will now describe how to construct $\left\langle q_{n}: n \in \omega\right\rangle$ and $\left\langle\dot{p}_{n}: n \in \omega\right\rangle$. $\stackrel{\circ}{0}_{0}$ is just $p_{0}$, the given condition. Since $p_{0} \in M \cap \mathbb{P}_{\gamma}$ and since $\gamma_{0} \in M$, $p_{0}\left\lceil\gamma_{0} \in M \cap \mathbb{P}_{\gamma_{0}}\right.$. As $\mathbb{P}_{\gamma_{0}}$ is proper, we may find an $\left(M, \mathbb{P}_{\gamma_{0}}\right)$ generic condition $q_{0} \leq p_{0}\left\lceil\gamma_{0}\right.$. Because $q_{0} \leq p_{0}\left\lceil\gamma_{0}, q_{0} \Vdash \gamma_{0} p_{0}\left\lceil\gamma_{0} \in \dot{G}_{\gamma_{0}}\right.\right.$. Now suppose that $q_{n}$ and $\stackrel{\circ}{p}_{n}$ are given to us. By clause $(1), q_{n}$ is $\left(M, \mathbb{P}_{\gamma_{n}}\right)$ generic, and by clause (2), $q_{n} \Vdash_{\gamma_{n}} \stackrel{\circ}{p}_{n} \in M \cap \mathbb{P}_{\gamma} \wedge \stackrel{\circ}{p}_{n}\left\lceil\gamma_{n} \in \stackrel{\circ}{G}_{\gamma_{n}}\right.$. Now this means that the hypothesis of 
Lemma 55 are satisfied by the iteration $\left\langle\mathbb{P}_{\alpha}, \AA_{\alpha}: \alpha \leq \gamma_{n+1}\right\rangle$, the elementary submodel $M$, the ordinal $\gamma_{n}$, the condition $q_{n}$ and by a $\mathbb{P}_{\gamma_{n}}$ name forced by $q_{n}$ to equal $\stackrel{p}{n}_{n}\left\lceil\gamma_{n+1}\right.$. So by Lemma 55 , we can find an $\left(M, \mathbb{P}_{\gamma_{n+1}}\right)$ generic condition $q_{n+1}$ such that:

(a) $q_{n+1}\left\lceil\gamma_{n}=q_{n}\right.$.

(b) $q_{n+1} \Vdash_{\gamma_{n+1}} \stackrel{\circ}{p}_{n}\left\lceil\gamma_{n+1} \in \stackrel{\circ}{G}_{\gamma_{n+1}}\right.$.

To find $\grave{p}_{n+1}$, we proceed as follows. Choose a $\left(\mathbf{V}, \mathbb{P}_{\gamma_{n+1}}\right)$ filter $G_{\gamma_{n+1}}$ with $q_{n+1} \in G_{\gamma_{n+1}}$. Since $\stackrel{p}{n}_{n}$ is a $\mathbb{P}_{\gamma_{n}}$ name, $\stackrel{\circ}{n}_{n}\left[G_{\gamma_{n+1}}\right]=\grave{p}_{n}\left[G_{\gamma_{n}}\right]$. Therefore $\grave{p}_{n}\left[G_{\gamma_{n+1}}\right] \in M \cap \mathbb{P}_{\gamma}$ and by (b) above, $\check{p}_{n}\left[G_{\gamma_{n+1}}\right]\left\lceil\gamma_{n+1} \in G_{\gamma_{n+1}}\right.$. Now, notice that $M\left[G_{\gamma_{n+1}}\right] \prec H(\theta)\left[G_{\gamma_{n+1}}\right]$, and that $H(\theta)\left[G_{\gamma_{n+1}}\right]$ is the same as $H(\theta)$ as computed within the universe $\mathbf{V}\left[G_{\gamma_{n+1}}\right]$. Observe also that both $\mathbb{P}_{\gamma} / G_{\gamma_{n+1}}$ and $f\left[G_{\gamma_{n+1}}\right]$ are elements of $M\left[G_{\gamma_{n+1}}\right]$. Thus we conclude that $\stackrel{\circ}{n}_{n}\left[G_{\gamma_{n+1}}\right] \in$ $\mathbb{P}_{\gamma} / G_{\gamma_{n+1}} \cap M\left[G_{\gamma_{n+1}}\right]$. Moreover, we see that $\Vdash_{\mathbb{P}_{\gamma} / G_{\gamma_{n+1}}} \stackrel{\circ}{f}\left[G_{\gamma_{n+1}}\right] \in \omega^{\omega}$. Thus by elementarity, we can find a sequence of conditions $\left\langle p^{i}: i \in \omega\right\rangle \in M\left[G_{\gamma_{n+1}}\right]$ and a function $f \in \omega^{\omega} \cap M\left[G_{\gamma_{n+1}}\right]$ such that the following hold:

(i) $p^{0}=\stackrel{\circ}{p}_{n}\left[G_{\gamma_{n+1}}\right]$ and $\forall i \in \omega\left[p^{i} \in \mathbb{P}_{\gamma} / G_{\gamma_{n+1}}\right]$.

(ii) $p^{i+1} \leq p^{i}$.

(iii) $\forall i \in \omega\left[p^{i} \Vdash_{\mathbb{P}_{\gamma} / G_{\gamma_{n+1}}} \stackrel{\circ}{f}\left[G_{\gamma_{n+1}}\right](i)=f(i)\right]$.

Recall that $q_{n+1}$ is an $\left(M, \mathbb{P}_{\gamma_{n+1}}\right)$ generic condition. Therefore, $M\left[G_{\gamma_{n+1}}\right] \cap$ $\mathbb{P}_{\gamma}=M \cap \mathbb{P}_{\gamma}$. It follows that $\left\langle p^{i}: i \in \omega\right\rangle \subset M$ (even though it is not an element of $M$ ). Now, since $\mathbb{P}_{\gamma_{n+1}}$ does not add eventually different reals, we can find $g \in \omega^{\omega} \cap M$ such that $|f \cap g|=\omega$. But we chose $S$ so that $\forall^{\infty} i \in \omega$ $[g(i) \in S(i)]$. Therefore, we can find $k \geq n$ such that $f(k) \in S(k)$. Now by (i)-(iii) above, $p^{k}$ has the following properties in $\mathbf{V}\left[G_{\gamma_{n+1}}\right]$ :

$$
\begin{aligned}
(+) & p^{k} \in M \cap \mathbb{P}_{\gamma} \text { and } p^{k}\left\lceil\gamma_{n+1} \in G_{\gamma_{n+1}} .\right. \\
(++) & p^{k} \leq \stackrel{\circ}{n}_{n}\left[G_{\gamma_{n+1}}\right] . \\
(+++) & p^{k} \mapsto_{\mathbb{P}_{\gamma} / G_{\gamma_{n+1}}} \exists k \geq n\left[\stackrel{\circ}{f}\left[G_{\gamma_{n+1}}\right](k) \in S(k)\right] .
\end{aligned}
$$

Since $G_{\gamma_{n+1}}$ was an arbitrary $\left(\mathbf{V}, \mathbb{P}_{\gamma_{n+1}}\right)$ generic filter containing $q_{n+1}$, we can use the maximal principle in $\mathbf{V}$ to end the proof by finding a $\mathbb{P}_{\gamma_{n+1}}$ name $\stackrel{\circ}{n+1}_{n+1}$ so that

$$
\begin{array}{ll}
(*) & q_{n+1} \Vdash_{\gamma_{n+1}} \stackrel{\circ}{p}_{n+1} \in M \cap \mathbb{P}_{\gamma} \wedge \stackrel{\circ}{p}_{n+1} \mid \gamma_{n+1} \in \stackrel{\circ}{G}_{\gamma_{n+1}} . \\
(* *) & q_{n+1} \Vdash_{\gamma_{n+1}} \stackrel{\circ}{n+1}_{n} \leq \\
(* * *) & q_{n+1} \Vdash_{\gamma_{n+1}} \stackrel{\circ}{p}_{n+1} \Vdash_{\mathbb{P}_{\gamma} / \stackrel{\circ}{G}_{\gamma_{n+1}}} \quad \exists k \geq n\left[\stackrel{\circ}{f}\left[\stackrel{\circ}{G}_{\gamma_{n+1}}\right](k) \in S(k)\right] .
\end{array}
$$

We will use Theorem 61 to show that the property of strongly preserving a strongly MAD family is preserved. Our proof of this will proceed by induction. However, just as in the case of the proof of the preservation of properness, we will have to make an inductive assumption that is stronger 
than simply the thing we want to prove. We state it below for the case of a two-step iteration.

Convention 62. In the context of the next lemma, in order to avoid unnecessary repetitions, we adopt the convention that for any poset $\mathbb{P}, \stackrel{\circ}{\mathbb{P}}_{\mathbb{P}}$ is the canonical $\mathbb{P}$ name for a $\mathbb{P}$ generic filter.

Lemma 63. Let $\mathscr{A} \subset \omega^{\omega}$ be a strongly MAD family. Let $\mathbb{P}$ be a poset which strongly preserves $\mathscr{A}$ and let $\mathbb{Q}$ be a $\mathbb{P}$ name for a poset such that $\mapsto_{\mathbb{P}} \mathbb{Q}$ strongly preserves $\mathscr{A}$. Suppose $M \prec H(\theta)$ is countable with $\mathscr{A}, \mathbb{P}, \mathbb{Q} \in M$. Fix $h \in \mathscr{A}$ that covers $M$ with respect to $\mathscr{A}$. Let $p \in \mathbb{P}$ and let $\stackrel{\circ}{r}$ be a $\mathbb{P}$ name such that:

(1) $p$ is $(M, \mathbb{P})$ generic.

(2) $p \Vdash_{\mathbb{P}} h$ covers $M\left[\stackrel{\circ}{G}_{\mathbb{P}}\right]$ with respect to $\mathscr{A}$.

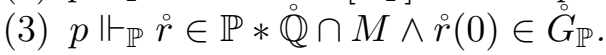

In this case, there is a $\stackrel{\mathrm{q}}{\in} \operatorname{dom}(\mathbb{Q})$ such that:

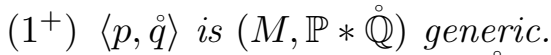

$\left(2^{+}\right)\langle p, \stackrel{\circ}{q}\rangle \Vdash_{\mathbb{P} * \mathbb{Q}} h$ covers $M[\stackrel{\circ}{\mathbb{P} * \mathbb{Q}}]$ with respect to $\mathscr{A}$.

$\left(3^{+}\right)\langle p, \stackrel{\circ}{q}\rangle \Vdash_{\mathbb{P} * \mathbb{Q}} \stackrel{\circ}{r} \in \stackrel{\circ}{G}_{\mathbb{P} * \mathbb{Q}}$.

Proof. Let $G_{\mathbb{P}}$ be a $(\mathbf{V}, \mathbb{P})$ generic filter with $p \in G_{\mathbb{P}}$. Within $\mathbf{V}\left[G_{P}\right]$, form $M\left[G_{\mathbb{P}}\right]$ and notice that $M\left[G_{\mathbb{P}}\right] \prec H(\theta)\left[G_{\mathbb{P}}\right]$ and that $H(\theta)\left[G_{\mathbb{P}}\right]$ is the same as $H(\theta)$ as computed within $\mathbf{V}\left[G_{\mathbb{P}}\right]$. Now, by assumption, $h$ covers $M\left[G_{\mathbb{P}}\right]$ with respect to $\mathscr{A}$. Also, $\mathscr{A}, \mathbb{Q}\left[G_{\mathbb{P}}\right] \in M\left[G_{\mathbb{P}}\right]$, and $\mathbb{Q}\left[G_{\mathbb{P}}\right]$ strongly preserves $\mathscr{A}$. Next, by assumption, $\dot{r}\left[G_{\mathbb{P}}\right] \in \mathbb{P} * \mathbb{Q} \cap M$. So there are $p^{\prime} \in \mathbb{P} \cap M$ and $\dot{q}^{\prime} \in \operatorname{dom}(\mathbb{Q}) \cap M$ such that $\dot{r}\left[G_{\mathbb{P}}\right](0)=p^{\prime}$ and $\dot{r}\left[G_{\mathbb{P}}\right](1)=\stackrel{q}{q}^{\prime}$. Moreover, $p^{\prime} \in G_{\mathbb{P}}$. It follows that $\stackrel{\circ}{q}^{\prime}\left[G_{\mathbb{P}}\right] \in \mathbb{Q}\left[G_{\mathbb{P}}\right] \cap M\left[G_{\mathbb{P}}\right]$. Thus, we may find a $q \leq \dot{q}^{\prime}\left[G_{\mathbb{P}}\right]$ such that in $\mathbf{V}\left[G_{\mathbb{P}}\right]$ :

(a) $q \in \mathbb{Q}\left[G_{\mathbb{P}}\right]$ is $\left(M\left[G_{\mathbb{P}}\right], \mathbb{Q}\left[G_{\mathbb{P}}\right]\right)$ generic.

(b) $q \Vdash_{\mathbb{Q}\left[G_{\mathbb{P}}\right]} h$ covers $M\left[G_{\mathbb{P}}\right]\left[\stackrel{\leftrightarrow}{G}_{\mathbb{Q}\left[G_{\mathbb{P}}\right]}\right]$ with respect to $\mathscr{A}$.

(c) $q \leq \grave{r}\left[G_{\mathbb{P}}\right](1)\left[G_{\mathbb{P}}\right]$.

Therefore, since $G_{\mathbb{P}}$ was an arbitrary $(\mathbf{V}, \mathbb{P})$ generic filter containing $p$, we may use the maximal principle in $\mathbf{V}$ to find $\stackrel{\circ}{q} \in \operatorname{dom}(\mathbb{Q})$ such that:

$\left(\mathrm{a}^{\prime}\right) p \Vdash_{\mathbb{P}} \stackrel{\circ}{q} \in \mathbb{Q}$ is $\left(M\left[\stackrel{\circ}{G}_{\mathbb{P}}\right], \stackrel{\mathbb{Q}}{)}\right)$ generic.

$\left(\mathrm{b}^{\prime}\right) p \Vdash_{\mathbb{P}} \stackrel{q}{q} \Vdash_{\mathbb{Q}} h$ covers $M\left[\dot{G}_{\mathbb{P}}\right]\left[\dot{G}_{\mathbb{Q}}\right]$ with respect to $\mathscr{A}$.

(c') $p \Vdash_{\mathbb{P}} \stackrel{\circ}{q} \leq \stackrel{\circ}{r}(1)\left[\stackrel{\circ}{G}_{\mathbb{P}}\right]$.

We will argue that $\stackrel{q}{q}$ is as needed. Indeed, by $\left(\mathrm{a}^{\prime}\right)$ above and by the fact that $p$ is $(M, \mathbb{P})$ generic, it easily follows that $\langle p, \stackrel{\circ}{q}\rangle$ is $(M, \mathbb{P} * \mathbb{Q})$ generic.

Next, we will argue that $\langle p, \stackrel{q}{\rangle}\rangle \Vdash_{\mathbb{P} * \mathbb{Q}} h$ covers $M\left[\dot{G}_{\mathbb{P} * \mathbb{Q}}\right]$ with respect to $\mathscr{A}$. Let $G_{\mathbb{P} * \mathbb{Q}}$ be a $(V, \mathbb{P} * \mathscr{Q})$ generic filter with $\langle p, \stackrel{q}{ }\rangle \in G_{\mathbb{P} * \mathbb{Q}}$. Notice that there 
is a $(\mathbf{V}, \mathbb{P})$ generic filter $G_{\mathbb{P}}$ and a $\left(\mathbf{V}\left[G_{\mathbb{P}}\right], \mathbb{Q}_{[}\left[G_{\mathbb{P}}\right]\right)$ generic filter $G_{\mathbb{Q}}\left[G_{\mathbb{P}}\right]$ such that in $\mathbf{V}\left[G_{\mathbb{P} * \mathbb{Q}}\right], G_{\mathbb{P} * \mathbb{Q}}=G_{\mathbb{P}} * G_{\mathbb{Q}\left[G_{\mathbb{P}}\right]}$. Moreover, $p \in G_{\mathbb{P}}$ and $\stackrel{q}{q}\left[G_{\mathbb{P}}\right] \in G_{\mathbb{Q}\left[G_{\mathbb{P}}\right]}$. Therefore in $\mathbf{V}\left[G_{\mathbb{P} * \mathbb{Q}}\right], h$ covers $M\left[G_{\mathbb{P}}\right]\left[G_{\mathbb{Q}\left[G_{\mathbb{P}}\right]}\right]$ with respect to $\mathscr{A}$. Now, let $f \in M\left[G_{\mathbb{P} * \mathbb{Q}}\right]$ be an infinite partial function avoiding $\mathscr{A}$. We can find a $\mathbb{P} * \mathbb{Q}$ name $f \in M$ with $f\left[G_{\mathbb{P} * \mathbb{Q}}\right]=f$. But we can think of $f$ as a $\mathbb{P}$ name for a $\mathbb{Q}$ name. So there is a $\mathbb{P}$ name $\stackrel{\circ}{f}\left[\stackrel{\circ}{G}_{\mathbb{P}}\right] \in M$ such that $\stackrel{\circ}{f}\left[G_{\mathbb{P}}\right]$ $\left[G_{\mathbb{Q}\left[G_{\mathbb{P}}\right]}\right]=\stackrel{\circ}{f}\left[G_{\mathbb{P} * \mathbb{Q}}\right]=f$. Thus $f\left[G_{\mathbb{P}}\right]$ is a $Q\left[G_{\mathbb{P}}\right]$ name in $M\left[G_{\mathbb{P}}\right]$ and so $f \in M\left[G_{\mathbb{P}}\right]\left[G_{\mathbb{Q}\left[G_{\mathbb{P}}\right]}\right]$. Therefore, $|h \cap f|=\omega$, as needed.

Finally, we must argue that $\langle p, \stackrel{\circ}{q}\rangle \Vdash_{\mathbb{P} * \mathbb{Q}} \stackrel{\circ}{r} \in \stackrel{\circ}{G}_{\mathbb{P} * \mathbb{Q}}$. Let $G_{\mathbb{P} * \mathbb{Q}}, G_{\mathbb{P}}$ and $G_{\mathbb{Q}\left[G_{\mathbb{P}}\right]}$ be as in the last paragraph. Once again, notice that since $\langle p, \stackrel{\circ}{q}\rangle \in$ $G_{\mathbb{P} * \mathbb{Q}}$, we have $p \in G_{\mathbb{P}}$ and $\stackrel{\circ}{q}\left[G_{\mathbb{P}}\right] \in G_{\mathbb{Q}\left[G_{\mathbb{P}}\right]}$. Notice also that since $\stackrel{\circ}{r}$ is a $\mathbb{P}$ name, $\stackrel{r}{r}\left[G_{\mathbb{P} * \mathbb{Q}}\right]=\stackrel{\circ}{r}\left[G_{\mathbb{P}}\right]$. Within $\mathbf{V}\left[G_{\mathbb{P}}\right]$, we have $\stackrel{r}{r}\left[G_{\mathbb{P}}\right]=\left\langle p^{\prime}, \stackrel{\circ}{q}^{\prime}\right\rangle \in \mathbb{P} * \mathbb{Q}$, where $p^{\prime} \in \mathbb{P}$ and $\stackrel{\circ}{q}^{\prime} \in \operatorname{dom}(\mathbb{Q})$. Also by $(3)$ above, $p^{\prime} \in G_{\mathbb{P}}$, and so $\stackrel{\circ}{q}^{\prime}\left[G_{\mathbb{P}}\right] \in \mathbb{Q}_{\mathbb{Q}}\left[G_{\mathbb{P}}\right]$. Moreover, by $\left(\mathrm{c}^{\prime}\right)$ above, $\stackrel{\circ}{q}\left[G_{\mathbb{P}}\right] \leq \stackrel{\circ}{q}^{\prime}\left[G_{\mathbb{P}}\right]$. Since $\stackrel{\stackrel{q}{q}\left[G_{\mathbb{P}}\right] \in G_{\mathbb{Q}}\left[G_{\mathbb{P}}\right]}{ }, \stackrel{\prime}{q}^{\prime}\left[G_{\mathbb{P}}\right] \in$ $G_{\mathbb{Q}\left[G_{\mathbb{P}}\right]}$ as well. Therefore, in $\mathbf{V}\left[G_{\mathbb{P} * \mathbb{Q}}\right]$, it follows that $\left\langle p^{\prime}, \dot{q}^{\prime}\right\rangle=\dot{r}\left[G_{\mathbb{P} * \mathbb{Q}}\right] \in$ $G_{\mathbb{P} * \mathbb{Q}}$, as required.

We will now prove the same for iterations of arbitrary length. We will make use of Theorem 61 in conjunction with the following, which is similar to Lemma 34.

Lemma 64. Let $\mathscr{A} \subset \omega^{\omega}$ be an infinite a.d. family and let $\mathbb{P}$ be a poset that does not add any eventually different reals. Suppose $\stackrel{f}{f}$ is a $\mathbb{P}$ name such that $\Vdash f$ is an infinite partial function avoiding $\mathscr{A}$. For each $p \in \mathbb{P}$, there is $q \leq p$ and an infinite partial function $f$ avoiding $\mathscr{A}$ such that for each $n \in \operatorname{dom}(f)$ there exists $r \leq q$ such that $r \Vdash n \in \operatorname{dom}(f) \wedge f^{\circ}(n)$ $=f(n)$.

Proof. We will use the well known fact that a poset which does not add eventually different reals does not make $\mathbf{V} \cap \omega^{\omega}$ meager (see proof of Lemma 2.4.8 in [3]). By Lemma 34, there is a countable set $\left\{\stackrel{\circ}{f}_{i}: i \in \omega\right\}$ of $\mathbb{P}$ names such that:

(1) $\Vdash \stackrel{\circ}{f}_{i} \subset \stackrel{\circ}{f}$ is an infinite partial function.

(2) $\Vdash \forall g \subset \omega \times \omega\left[\right.$ if $\forall i \in \omega\left[\left|g \cap \dot{f}_{i}\right|=\omega\right]$, then $g$ avoids $\left.\mathscr{A}\right]$.

Fix a condition $p \in \mathbb{P}$. As $\mathbb{P}$ does not make $\mathbf{V} \cap \omega^{\omega}$ meager, there are $q \leq p$ and $h \in \omega^{\omega}$ such that for each $i \in \omega, q \Vdash\left|h \cap \dot{f}_{i}\right|=\omega$. Now, set $X=$ $\{n \in \omega: \exists r \leq q[r \Vdash n \in \operatorname{dom}(\dot{f}) \wedge h(n)=\dot{f}(n)]\}$ and set $f=h\lceil X$. To finish the proof, by (2) above, it is enough to check that for each $i \in \omega$, $q \Vdash\left|f \cap \dot{\circ}_{i}\right|=\omega$. Indeed, suppose $r \leq q$ and $k \in \omega$. Since $q \Vdash\left|h \cap \stackrel{\circ}{f}_{i}\right|=\omega$, there are $s \leq r$ and $n>k$ such that $s \Vdash n \in \operatorname{dom}\left(f_{i}\right) \wedge h(n)=\dot{\circ}_{i}(n)$. But 
since $\Vdash \stackrel{\circ}{f}_{i} \subset \stackrel{\circ}{f}$, it follows that $s \Vdash n \in \operatorname{dom}(\stackrel{\circ}{)} \wedge h(n)=\stackrel{\circ}{f}(n)$, which means that $n \in \operatorname{dom}(f)$.

Theorem 65. Let $\mathscr{A} \subset \omega^{\omega}$ be a strongly MAD family. Let $\left\langle\mathbb{P}_{\alpha}, \AA_{\mathbb{Q}}\right.$ : $\alpha \leq \gamma\rangle$ be a $C S$ iteration such that $\forall \alpha<\gamma\left[\Vdash_{\alpha} \mathbb{Q}_{\alpha}\right.$ strongly preserves $\left.\mathscr{A}\right]$. Let $M \prec H(\theta)$ be countable with $\left\langle\mathbb{P}_{\alpha}, \AA_{\alpha}: \alpha \leq \gamma\right\rangle, \mathscr{A} \in M$. Let $h \in \mathscr{A}$ cover $M$ with respect to $\mathscr{A}$. Suppose $\gamma_{0} \in \gamma \cap M$ and let $q_{0} \in \mathbb{P}_{\gamma_{0}}$ be an $\left(M, \mathbb{P}_{\gamma_{0}}\right)$ generic condition such that $q_{0} \Vdash_{\gamma_{0}} h$ covers $M\left[\dot{G}_{\gamma_{0}}\right]$ with respect

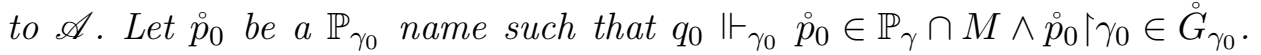
There is a $q \in \mathbb{P}_{\gamma}$ with $q\left\lceil\gamma_{0}=q_{0}\right.$, which is $\left(M, \mathbb{P}_{\gamma}\right)$ generic, such that $q \Vdash_{\gamma} h$ covers $M\left[\dot{G}_{\gamma}\right]$ with respect to $\mathscr{A}$ and $q \Vdash_{\gamma} \stackrel{\circ}{0}_{0} \in \stackrel{\leftrightarrow}{G}_{\gamma}$. In particular, $\mathbb{P}_{\gamma}$ strongly preserves $\mathscr{A}$.

Proof. Before proving the main claim of the theorem, we remark that the last sentence of the theorem easily follows from the main claim. To see this, suppose $p_{0} \in \mathbb{P}_{\gamma} \cap M$ is a condition. Now, apply the main claim of the theorem with $\gamma_{0}=0$, the trivial condition as $q_{0}$, and $\dot{p}_{0}=p_{0}$.

The proof of the main claim is by induction on $\gamma$. Let us assume that the theorem holds for all $\alpha<\gamma$. The case when $\gamma$ is a successor has already been dealt with in Lemma 63. So we assume that $\gamma$ is a limit ordinal. We observe that it follows from our inductive hypothesis that no $\mathbb{P}_{\alpha}$ adds eventually different reals, for $\alpha<\gamma$. As $\gamma$ is a limit ordinal, it follows from Theorem 61 that $\mathbb{P}_{\gamma}$ does not add an eventually different real. We will make use of this observation in what follows.

Put $\gamma^{\prime}=\sup (\gamma \cap M)$ and let $\left\langle\gamma_{n}: n \in \omega\right\rangle \subset M \cap \gamma$ be an increasing sequence that is cofinal in $\gamma^{\prime}$. Let $\left\langle D_{n}: n \in \omega\right\rangle$ enumerate all the dense open subsets of $\mathbb{P}_{\gamma}$ that are elements of $M$. Also, we let $\left\langle f_{i}^{\circ}: i \in \omega\right\rangle$ enumerate all $\mathbb{P}_{\gamma}$ names in $M$ such that $\Vdash_{\gamma} \stackrel{\circ}{i}_{i}$ is an infinite partial function avoiding $\mathscr{A}$. We will build two sequences $\left\langle q_{n}: n \in \omega\right\rangle$ and $\left\langle\dot{p}_{n}: n \in \omega\right\rangle$ such that the following hold:

(1) $q_{0}$ is given, $q_{n} \in \mathbb{P}_{\gamma_{n}}, q_{n}$ is $\left(M, \mathbb{P}_{\gamma_{n}}\right)$ generic, and $q_{n+1}\left\lceil\gamma_{n}=q_{n}\right.$.

(2) $q_{n} \Vdash_{\gamma_{n}} h$ covers $M\left[{\stackrel{\circ}{\gamma_{n}}}_{n}\right]$ with respect to $\mathscr{A}$.

(3) $\stackrel{\circ}{0}_{0}=p_{0}, \stackrel{\circ}{n}_{n} \in \mathbf{V}^{\mathbb{P}_{\gamma_{n}}}$, and $q_{n} \Vdash_{\gamma_{n}} \stackrel{\circ}{p}_{n} \in M \cap \mathbb{P}_{\gamma} \wedge \stackrel{\circ}{n}_{n}\left\lceil\gamma_{n} \in{\stackrel{\circ}{\gamma_{n}}}_{\text {. }}\right.$.

(4) $q_{n+1} \Vdash_{\gamma_{n+1}} \stackrel{\circ}{n+1}_{n+1} \in D_{n} \wedge \grave{p}_{n+1} \leq \grave{p}_{n}$.

(5) $\forall i \leq n\left[q_{n+1} \Vdash_{\gamma_{n+1}} \Phi_{i}\right]$, where $\Phi_{i}$ is this formula in $\mathbb{P}_{\gamma_{n+1}}$ forcing language: $\stackrel{\circ}{p+1}_{n+1} \Vdash_{\mathbb{P}_{\gamma} / \stackrel{\leftrightarrow}{G}_{\gamma_{n+1}}} \exists k_{i}^{n} \geq n\left[k_{i}^{n} \in \operatorname{dom}\left(\stackrel{\circ}{f}_{i}\left[\stackrel{\leftrightarrow}{G}_{\gamma_{n+1}}\right]\right) \wedge f_{i}\left[{\stackrel{\circ}{\gamma_{n+1}}}_{\gamma_{n+1}}\right]\left(k_{i}^{n}\right)\right.$ $\left.=h\left(k_{i}^{n}\right)\right]$.

Before describing how to construct such sequences, we will argue that it is enough to do so. Put $q=\left(\bigcup q_{n}\right)^{\frown} \mathbb{1} \in \mathbb{P}_{\gamma}$. By Lemma 54, $\forall n \in \omega$ $\left[q \Vdash_{\gamma} \stackrel{\circ}{p}_{n} \in \stackrel{\circ}{G}_{\gamma}\right]$. We will first argue that $q$ is $\left(M, \mathbb{P}_{\gamma}\right)$ generic. It suffices to show that for each $n \in \omega, q \Vdash_{\gamma} D_{n} \cap M \cap \stackrel{\circ}{G}_{\gamma} \neq 0$. But by clauses (3) and (4) 
and by Lemma 54, it is clear that $q \Vdash_{\gamma} \stackrel{\circ}{n+1}_{n+1} \in D_{n} \cap M \cap \stackrel{\circ}{G}_{\gamma}$. That $q \Vdash_{\gamma} h$ covers $M\left[\stackrel{\circ}{G}_{\gamma}\right]$ with respect to $\mathscr{A}$ will be verified next.

We will first argue that it is sufficient to show that $\forall i \in \omega\left[q \Vdash_{\gamma}\left|h \cap \stackrel{\circ}{f}_{i}\right|\right.$ $=\omega]$. Assume this and let $G_{\gamma}$ be a $\left(\mathbf{V}, \mathbb{P}_{\gamma}\right)$ generic filter with $q \in G_{\gamma}$. Let $f \in M\left[G_{\gamma}\right]$ be an infinite partial function avoiding $\mathscr{A}$. There is a $\mathbb{P}_{\gamma}$ name $\stackrel{\circ}{f} \in M$ such that $f\left[G_{\gamma}\right]=f$. But by elementarity of $M$, we can find such an $f$ with the additional property that $\vdash_{\gamma} \stackrel{\circ}{f}$ is an infinite partial function avoiding $\mathscr{A}$. Thus, $f=\stackrel{\circ}{f}_{i}\left[G_{\gamma}\right]$, for some $i \in \omega$, and so $|h \cap f|=\omega$.

We will now check that $\forall i \in \omega\left[q \Vdash_{\gamma}\left|h \cap \stackrel{\circ}{f}_{i}\right|=\omega\right]$. Fix $i \in \omega$. Let $r \leq q$ and let $m \in \omega$. Choose a $\left(\mathbf{V}, \mathbb{P}_{\gamma}\right)$ generic filter with $r \in G_{\gamma}$. Choose $n \in \omega$ with $m, i \leq n$. By Lemma 58, we know that $G_{\gamma}$ is $\left(\mathbf{V}\left[G_{\gamma_{n+1}}\right], \mathbb{P}_{\gamma} / G_{\gamma_{n+1}}\right)$ generic and that in $\mathbf{V}\left[G_{\gamma}\right], G_{\gamma}=G_{\gamma_{n+1}} * G_{\gamma}$ holds. Notice that $q \in G_{\gamma}$, and therefore $q_{n+1} \in G_{\gamma_{n+1}}$. Also, since $\stackrel{\circ}{p+1}_{n+1}$ is a $\mathbb{P}_{\gamma_{n+1}}$ name, $\stackrel{\circ}{n+1}_{n+1}\left[G_{\gamma}\right]=$ $\stackrel{\circ}{p+1}_{n}\left[G_{\gamma_{n+1}}\right]$. It follows from clauses (3) and (5) that $\stackrel{\circ}{n+1}_{n+1}\left[G_{\gamma}\right] \in M \cap \mathbb{P}_{\gamma}$, that $\stackrel{\circ}{p}_{n+1}\left[G_{\gamma}\right]\left\lceil\gamma_{n+1} \in G_{\gamma_{n+1}}\right.$, and that in $\mathbf{V}\left[G_{\gamma_{n+1}}\right], \stackrel{\circ}{p}_{n+1}\left[G_{\gamma}\right] \Vdash_{\mathbb{P}_{\gamma} / G_{\gamma_{n+1}}}$ $\exists k_{i}^{n} \geq n\left[k_{i}^{n} \in \operatorname{dom}\left(\dot{\circ}_{i}\left[G_{\gamma_{n+1}}\right]\right) \wedge \dot{\circ}_{i}\left[G_{\gamma_{n+1}}\right]\left(k_{i}^{n}\right)=h\left(k_{i}^{n}\right)\right]$. On the other hand, we know from Lemma 54 that $\stackrel{\circ}{n+1}_{n+1}\left[G_{\gamma}\right] \in G_{\gamma}$. Therefore, in $\mathbf{V}\left[G_{\gamma}\right]$, we are able to find a $k_{i}^{n} \geq n \geq m$ such that $k_{i}^{n} \in \operatorname{dom}\left(\AA_{i}\left[G_{\gamma_{n+1}}\right]\left[G_{\gamma}\right]\right)$ and $\stackrel{\circ}{f}_{i}\left[G_{\gamma_{n+1}}\right]\left[G_{\gamma}\right]\left(k_{i}^{n}\right)=h\left(k_{i}^{n}\right)$. However, $\stackrel{\circ}{i}_{i}\left[G_{\gamma_{n+1}}\right]\left[G_{\gamma}\right]=\stackrel{\circ}{f}\left[_{i}\left[G_{\gamma}\right]\right.$. As $r \in G_{\gamma}$, there is $s \leq r$ such that $s \Vdash_{\gamma} k_{i}^{n} \in \operatorname{dom}\left(\dot{f}_{i}\right) \wedge \dot{\circ}_{i}\left(k_{i}^{n}\right)=h\left(k_{i}^{n}\right)$. As $k_{i}^{n} \geq m$, this is as needed.

Next we describe how to construct $\left\langle q_{n}: n \in \omega\right\rangle$ and $\left\langle\dot{p}_{n}: n \in \omega\right\rangle$. $q_{0}$ and $\dot{p}_{0}$ are both given to us. Now assume that $q_{n}$ and $\dot{p}_{n}$ are given. We can apply the inductive hypothesis to the iteration $\left\langle\mathbb{P}_{\alpha}, \varrho_{\alpha}: \alpha \leq \gamma_{n+1}\right\rangle$, the elementary submodel $M$, the ordinal $\gamma_{n}$, the condition $q_{n}$ and a $\mathbb{P}_{\gamma_{n}}$ name forced by $q_{n}$ to equal $\stackrel{\circ}{n}_{n}\left\lceil\gamma_{n+1}\right.$ to find an $\left(M, \mathbb{P}_{\gamma_{n+1}}\right)$ generic condition $q_{n+1}$ such that:

(a) $q_{n+1}\left\lceil\gamma_{n}=q_{n}\right.$

(b) $q_{n+1} \Vdash_{\gamma_{n+1}} h$ covers $M\left[\stackrel{\circ}{G}_{\gamma_{n+1}}\right]$ with respect to $\mathscr{A}$.

(c) $q_{n+1} \Vdash_{\gamma_{n+1}} \stackrel{\circ}{p}_{n}\left\lceil\gamma_{n+1} \in \stackrel{\circ}{G}_{\gamma_{n+1}}\right.$.

To find $\stackrel{\circ}{n+1}_{n+1}$ we proceed as follows. Let $G_{\gamma_{n+1}}$ be a $\left(\mathbf{V}, \mathbb{P}_{\gamma_{n+1}}\right)$ generic filter with $q_{n+1} \in G_{\gamma_{n+1}}$. We begin with some general observations. Note that $\check{p}_{n}\left[G_{\gamma_{n+1}}\right] \in \mathbb{P}_{\gamma} \cap M$. Also, $\grave{p}_{n}\left[G_{\gamma_{n+1}}\right]\left\lceil\gamma_{n+1} \in G_{\gamma_{n+1}}\right.$. Thus we conclude that $\stackrel{\circ}{p}_{n}\left[G_{\gamma_{n+1}}\right]$ is a condition in $\mathbb{P}_{\gamma} / G_{\gamma_{n+1}} \cap M\left[G_{\gamma_{n+1}}\right]$. Moreover, $\mathbb{P}_{\gamma} / G_{\gamma_{n+1}} \in$ $M\left[G_{\gamma_{n+1}}\right]$.

Now, we will describe how to take care of the dense open set $D_{n}$. We make use of the fact that if $\pi: \mathbb{Q} \rightarrow \mathbb{P}$ is a projection and $D \subset \mathbb{Q}$ is dense, and if $G \subset \mathbb{P}$ is a $(\mathbf{V}, \mathbb{P})$ generic filter, then in $\mathbf{V}[G], D / G=D \cap$ $\mathbb{Q} / G$ is dense in $\mathbb{Q} / G$. Applying this to $\mathbb{P}_{\gamma}, \mathbb{P}_{\gamma_{n+1}}$ and $D_{n}$, we conclude 
that $D_{n} \cap \mathbb{P}_{\gamma} / G_{\gamma_{n+1}}$ is dense in $\mathbb{P}_{\gamma} / G_{\gamma_{n+1}}$. Since $D_{n}, \stackrel{\circ}{p}_{n}\left[G_{\gamma_{n+1}}\right] \in M\left[G_{\gamma_{n+1}}\right]$, we can find a $p^{0} \in D_{n} \cap \mathbb{P}_{\gamma} / G_{\gamma_{n+1}} \cap M\left[G_{\gamma_{n+1}}\right]$ such that $p^{0} \leq \grave{p}_{n}\left[G_{\gamma_{n+1}}\right]$. We note here that since $D_{n}$ is open, any further extension of $p^{0}$ will stay within $D_{n}$.

Next, we describe how to deal with $f_{0}^{\circ}\left[G_{\gamma_{n+1}}\right]$. First of all, since in the ground model $\mathbf{V}, \Vdash_{\gamma} f_{0}$ is an infinite partial function avoiding $\mathscr{A}$, we see that in $\mathbf{V}\left[G_{\gamma_{n+1}}\right], \Vdash_{\mathbb{P}_{\gamma} / G_{\gamma_{n+1}}} f_{0}^{\circ}\left[G_{\gamma_{n+1}}\right]$ is an infinite partial function avoiding $\mathscr{A}$. Moreover, we have observed above that $\mathbb{P}_{\gamma}$ does not add eventually different reals. As $\mathbb{P}_{\gamma}$ is forcing equivalent to $\mathbb{P}_{\gamma_{n+1}} * \mathbb{P}_{\gamma} / \dot{G}_{\gamma_{n+1}}$, it follows that $\mathbb{P}_{\gamma} / G_{\gamma_{n+1}}$ does not add eventually different reals over $\mathbf{V}\left[G_{\gamma_{n+1}}\right]$. As $\dot{f}_{0}\left[G_{\gamma_{n+1}}\right] \in M\left[G_{\gamma_{n+1}}\right]$, we can apply Lemma 64 to $\mathbb{P}_{\gamma} / G_{\gamma_{n+1}}$ to find a $\tilde{p}^{0} \in \mathbb{P}_{\gamma} / G_{\gamma_{n+1}} \cap M\left[G_{\gamma_{n+1}}\right]$ with $\tilde{p}^{0} \leq p^{0}$ and an infinite partial function $f \in M\left[G_{\gamma_{n+1}}\right]$ as in the lemma which avoids $\mathscr{A}$. But by (b) above, $h$ covers $M\left[G_{\gamma_{n+1}}\right]$ with respect to $\mathscr{A}$. Therefore, $|h \cap f|=\omega$.

Choose $k_{0}^{n} \geq n$ such that $k_{0}^{n} \in \operatorname{dom}(f)$ and $h(n)=f(n)$. By the lemma, there is a $p^{1} \leq \tilde{p}^{0}$ in $\mathbb{P}_{\gamma} / G_{\gamma_{n+1}} \cap M\left[G_{\gamma_{n+1}}\right]$ such that $p^{1} \Vdash_{\mathbb{P}_{\gamma} / G_{\gamma_{n+1}}} k_{0}^{n} \in$ $\operatorname{dom}\left(\stackrel{\circ}{0}_{0}\left[G_{\gamma_{n+1}}\right]\right) \wedge \stackrel{\circ}{f}_{0}\left[G_{\gamma_{n+1}}\right]\left(k_{0}^{n}\right)=f\left(k_{0}^{n}\right)=h\left(k_{0}^{n}\right)$. Repeating this argument another $n$ times we get $p^{n+1} \in \mathbb{P}_{\gamma} / G_{\gamma_{n+1}} \cap M\left[G_{\gamma_{n+1}}\right]$ with $p^{n+1} \leq \dot{p}_{n}\left[G_{\gamma_{n+1}}\right]$ as well as numbers $k_{i}^{n} \geq n$ for each $i \leq n$ so that for each such $i$, we have $p^{n+1} \Vdash_{\mathbb{P}_{\gamma} / G_{\gamma_{n+1}}} k_{i}^{n} \in \operatorname{dom}\left(\stackrel{\circ}{f}_{i}\left[G_{\gamma_{n+1}}\right]\right) \wedge \stackrel{\circ}{f}_{i}\left[G_{\gamma_{n+1}}\right]\left(k_{i}^{n}\right)=h\left(k_{i}^{n}\right)$. Now, we note that since $q_{n+1}$ is an $\left(M, \mathbb{P}_{\gamma_{n+1}}\right)$ generic condition and $q_{n+1} \in G_{\gamma_{n+1}}$, $M\left[\mathbb{P}_{\gamma_{n+1}}\right] \cap \mathbb{P}_{\gamma}=M \cap \mathbb{P}_{\gamma}$. Therefore, $p^{n+1}$ is in fact in $M$.

Thus we have found a condition $p^{n+1}$ with the following properties:

(i) $p^{n+1} \in \mathbb{P}_{\gamma} \cap M$ and $p^{n+1}\left\lceil\gamma_{n+1} \in G_{\gamma_{n+1}}\right.$.

(ii) $p^{n+1} \leq \stackrel{\circ}{p}_{n}\left[G_{\gamma_{n+1}}\right]$ and $p^{n+1} \in D_{n}$.

(iii) $\forall i \leq n\left[p^{n+1} \Vdash_{\mathbb{P}_{\gamma} / G_{\gamma_{n+1}}} \Phi_{i}\right]$, where $\Phi_{i}$ is the formula

$$
\exists k_{i}^{n} \geq n\left[k_{i}^{n} \in \operatorname{dom}\left(\AA_{i}\left[G_{\gamma_{n+1}}\right]\right) \wedge \dot{\circ}_{i}\left[G_{\gamma_{n+1}}\right]\left(k_{i}^{n}\right)=h\left(k_{i}^{n}\right)\right] .
$$

Since $G_{\gamma_{n+1}}$ was an arbitrary $\left(\mathbf{V}, \mathbb{P}_{\gamma_{n+1}}\right)$ generic filter containing $q_{n+1}$ we can use the maximal principle in $\mathbf{V}$ to find a $\mathbb{P}_{\gamma_{n+1}}$ name $\dot{p}_{n+1}$ such that:

(i') $q_{n+1} \Vdash \Vdash_{\gamma_{n+1}}{\stackrel{\circ}{p_{n+1}}}_{\stackrel{p}{p}} \in \mathbb{P}_{\gamma} \cap M \wedge \stackrel{\circ}{p}_{n+1}\left\lceil\gamma_{n+1} \in \stackrel{\circ}{G}_{\gamma_{n+1}}\right.$.

(ii') $q_{n+1} \Vdash_{\gamma_{n+1}} \stackrel{\circ}{p+1}_{n+1} \leq \stackrel{\circ}{p}_{n} \wedge \stackrel{\circ}{p+1}_{n+1} \in D_{n}$.

(iii') $\forall i \leq n\left[q_{n+1} \Vdash_{\gamma_{n+1}} \Phi_{i}\right]$, where $\Phi_{i}$ is the formula

$$
\stackrel{\circ}{p}_{n+1} \Vdash_{\mathbb{P}_{\gamma} / \stackrel{\leftrightarrow}{G}_{\gamma_{n+1}}} \exists k_{i}^{n} \geq n\left[k_{i}^{n} \in \operatorname{dom}\left(\stackrel{\circ}{f}_{i}\left[{\stackrel{\circ}{\gamma_{n+1}}}_{\gamma_{n+1}}\right]\right) \wedge \stackrel{\circ}{f}_{i}\left[{\stackrel{\circ}{\gamma_{n+1}}}_{\gamma_{n+1}}\right]\left(k_{i}^{n}\right)=h\left(k_{i}^{n}\right)\right] .
$$

Corollary 66. Let $\mathscr{A} \subset \omega^{\omega}$ be a strongly MAD family. If $\left\langle\mathbb{P}_{\alpha}, \mathbb{Q}_{\alpha}\right.$ : $\alpha \leq \gamma\rangle$ is a CS iteration such that $\forall \alpha<\gamma\left[\Vdash_{\alpha} \mathbb{Q}_{\alpha}\right.$ has diagonal fusion $]$, then $\mathbb{P}_{\gamma}$ strongly preserves $\mathscr{A}$. In particular, if $\forall \alpha<\gamma\left[\Vdash_{\alpha} \mathbb{Q}_{\alpha}=\mathbb{M} \vee \AA_{\alpha}=\mathbb{S}\right]$, then $\mathbb{P}_{\gamma}$ strongly preserves $\mathscr{A}$. 
7. It is consistent that there are no strongly MAD families of size $\mathfrak{c}$. In this section we will show that it is consistent that there are no strongly MAD families of size continuum. In fact, we will prove something more general. In Section 5 we showed that strongly MAD families are Cohen-indestructible. It is possible to combine this fact with an elementary submodel argument to show that there are no strongly MAD families of size continuum in the Cohen model. Here by the Cohen model we mean the model one gets by adding $\aleph_{2}$ Cohen reals to a ground model satisfying $\mathrm{CH}$. Arguments like this are well known in the literature. For example, Kunen used an argument of this sort to show that there are no well ordered chains of length $\omega_{2}$ in $\mathcal{P}(\omega) /$ Fin in the Cohen model (see Proposition 7.1 of [5]). To apply such an argument to strongly MAD families, we proceed by contradiction. We fix $\mathscr{A}$, a nice $\operatorname{Fn}\left(\omega_{2}, 2\right)$ name for a strongly MAD family of size $\aleph_{2}$, and we take a countably closed elementary submodel $M$ in the ground model of size $\aleph_{1}$ with $\mathscr{A} \in M$. Now, if $\delta=M \cap \omega_{2}$, then $M \cap \mathscr{A}$ is a nice $\operatorname{Fn}(\delta, 2)$ name for a strongly MAD family of size $\aleph_{1}$. Since strongly MAD families are Cohen-indestructible, $M \cap \mathscr{A}$ still names a MAD family in the final model, a contradiction.

Juhász and others (for example, see [12], [11], [10], [6]) have introduced a set of axioms that attempt to capture the combinatorics of $\mathcal{P}(\omega)$ and $\omega^{\omega}$ in the Cohen model. These axioms fall into two categories. There are axioms of the elementary submodel kind, which say that there are elementary submodels of size $\aleph_{1}$ that "capture" $\mathcal{P}(\omega)$. The other kind are homogeneity axioms, which say that given any sequence $\left\langle r_{\alpha}: \alpha<\omega_{2}\right\rangle$ of reals, there are $\aleph_{2}$ of them that "look alike". It is of interest to see whether any of these axioms imply the above mentioned result about strongly MAD families. It is natural to try to do this from one of the elementary submodel axioms. We show in this section that the weak Freese-Nation property of $\mathcal{P}(\omega)(\operatorname{wFN}(\mathcal{P}(\omega)))$ implies that all strongly MAD families have size at most $\aleph_{1}$. It is shown in [6] that $\operatorname{wFN}(\mathcal{P}(\omega))$ holds in any model gotten by adding fewer than $\aleph_{\omega}$ Cohen reals to a ground model satisfying $\mathrm{CH}$. The usual definition of ${ }_{\mathrm{wFN}}(\mathcal{P}(\omega))$ is in terms of weak Freese-Nation maps. It is shown in [6] that the definition we adopt below is equivalent to this usual definition.

Definition 67 . Given $B \subset \mathcal{P}(\omega)$, we write $B \leq_{\sigma} \mathcal{P}(\omega)$ to mean that for all $a \in \mathcal{P}(\omega)$, there is a countable set $C \subset B \cap \mathcal{P}(a)$ such that $\forall b \in B \cap \mathcal{P}(a)$ $\exists c \in C[b \subset c \subset a]$.

Definition 68. $\mathrm{wFN}(\mathcal{P}(\omega))$ is the following statement: Whenever $\theta$ is a sufficiently large regular cardinal, and $N \prec H(\theta)$ with $\omega_{1} \subset N$, then $N \cap \mathcal{P}(\omega) \leq_{\sigma} \mathcal{P}(\omega)$.

While it is possible to prove our result by arguing only with strongly MAD families of functions, the proof is a bit smoother if we argue instead 
with strongly MAD families of sets. The notion of a strongly MAD family of subsets of $\omega$ was introduced in Malykhin [19]. It has been further studied by Kurilić [18] and Hrušák and García Ferreira [9]. The definition of this concept is identical to our Definition 2, but with $\omega^{\omega}$ replaced everywhere by $[\omega]^{\omega}$, and with the additional requirement that the family be infinite.

Definition 69. Let $X$ be a countable set. Two sets $a, b \in[X]^{\omega}$ are a.d. if $a \cap b$ is finite. A family $\mathscr{A} \subset[X]^{\omega}$ is a.d. if its members are pairwise a.d. An a.d. family $\mathscr{A} \subset[X]^{\omega}$ is $M A D$ if for every $b \in[X]^{\omega}$ there is some $a \in \mathscr{A}$ such that $|a \cap b|=\omega$. Note that we are allowing finite families to be MAD.

Definition 70. Let $X$ be a countable set, and let $\mathscr{A} \subset[X]^{\omega}$ be an a.d. family. We say $b \in[X]^{\omega}$ avoids $\mathscr{A}$ if for every finite set $\left\{a_{0}, \ldots, a_{k}\right\} \subset \mathscr{A}$, $\left|b \backslash\left(a_{0} \cup \cdots \cup a_{k}\right)\right|=\omega$. We say that $\mathscr{A}$ is strongly $M A D$ in $[X]^{\omega}$ if $\mathscr{A}$ is an infinite family and if for any countable family $\left\{b_{i}: i \in \omega\right\} \subset[X]^{\omega}$ of sets avoiding $\mathscr{A}$, there is $a \in \mathscr{A}$ such that $\forall i \in \omega\left[\left|a \cap b_{i}\right|=\omega\right]$.

A connection between strongly MAD families of functions in $\omega^{\omega}$ and strongly MAD families of sets is given by the following.

LEMma 71. If there is a strongly MAD family in $\omega^{\omega}$, then there is a strongly MAD family in $[\omega]^{\omega}$ which is of the same size.

Proof. Let $\mathscr{A} \subset \omega^{\omega}$ be strongly MAD. For each $n \in \omega$, let $C_{n}$ be the $n$th vertical column of $\omega \times \omega$. That is, $C_{n}=\{\langle n, m\rangle: m \in \omega\}$. It is clear that each $C_{n}$ is a.d. from $\mathscr{A}$. Thus $\mathscr{A} \cup\left\{C_{n}: n \in \omega\right\}$ is an infinite a.d. family in $[\omega \times \omega]^{\omega}$ and it has the same cardinality as $\mathscr{A}$. We will argue that it is strongly MAD in $[\omega \times \omega]^{\omega}$. Let $\left\{A_{n}: n \in \omega\right\} \subset[\omega \times \omega]^{\omega}$ be a countable family avoiding $\mathscr{A} \cup\left\{C_{n}: n \in \omega\right\}$. We will find infinite partial functions $f_{n} \subset A_{n}$ avoiding $\mathscr{A}$. The argument is similar to the proof of Lemma 21 .

We will first argue that $A_{n}$ has infinite intersection with infinitely many members of $\mathscr{A}$. Suppose this is false. Fix $\left\{h_{0}, \ldots, h_{k}\right\} \subset \mathscr{A}$ such that for any $h \in \mathscr{A}$, if $\left|h \cap A_{n}\right|=\omega$, then $h=h_{i}$ for some $0 \leq i \leq k$. Put $B=$ $A_{n} \backslash\left(h_{0} \cup \cdots \cup h_{k}\right)$. Our assumption implies that $B$ is a.d. from $\mathscr{A}$. Therefore, since strongly MAD families are van Douwen MAD, it follows that there is no infinite partial function $p \subset B$. Thus for all but finitely many $n \in \omega$, $C_{n} \cap B=0$. But then there is $n \in \omega$ such that $B \subset C_{0} \cup \cdots \cup C_{n}$, whence $A_{n} \subset h_{0} \cup \cdots \cup h_{k} \cup C_{0} \cup \cdots \cup C_{n}$, contradicting our assumption that $A_{n}$ avoids $\mathscr{A} \cup\left\{C_{n}: n \in \omega\right\}$.

Hence we can fix an infinite set $\left\{h_{i}: i \in \omega\right\} \subset \mathscr{A}$ such that $\forall i \in \omega$ $\left[\left|h_{i} \cap A_{n}\right|=\omega\right]$. Now, put $p_{i}=h_{i} \cap A_{n}$. This is an infinite partial function. It is possible to choose infinite partial functions $g_{i} \subset p_{i}$ such that $\forall i<j<\omega$ $\left[\operatorname{dom}\left(g_{i}\right) \cap \operatorname{dom}\left(g_{j}\right)=0\right]$. Put $f_{n}=\bigcup g_{i}$. This is an infinite partial function and clearly $f_{n} \subset A_{n}$. Moreover, $f_{n}$ has infinite intersection with infinitely many members of $\mathscr{A}$. So $f_{n}$ avoids $\mathscr{A}$. Thus $\left\{f_{n}: n \in \omega\right\}$ is a countable 
family of infinite partial functions avoiding $\mathscr{A}$. So by Lemma 7 we can find $h \in \mathscr{A}$ such that $\forall n \in \omega\left[\left|h \cap f_{n}\right|=\omega\right]$. But since $f_{n} \subset A_{n}$, we conclude that $\forall n \in \omega\left[\left|h \cap A_{n}\right|=\omega\right]$.

We do not know if the converse is true:

QUESTION 72. Suppose that there is a strongly MAD family in $[\omega]^{\omega}$. Is there a strongly MAD family in $\omega^{\omega}$ ?

Lemma 71 tells us that every strongly MAD family of functions in $\omega^{\omega}$ gives rise to a strongly MAD family of the same size in $[\omega]^{\omega}$. Thus if we show that ${ } \mathrm{FN}(\mathcal{P}(\omega))$ implies that every strongly MAD family in $[\omega]^{\omega}$ has size at most $\aleph_{1}$, then the same will hold for strongly MAD families of functions as well.

Given a strongly MAD family $\mathscr{A} \subset[\omega]^{\omega}$, we will apply ${ }{ }^{2} \mathrm{FN}(\mathcal{P}(\omega))$ to an elementary submodel $N \prec H(\theta)$ which is "AA-covering" in the following sense.

Definition 73. Let $\mathscr{A} \subset[\omega]^{\omega}$ be a strongly MAD family, and let $N \prec$ $H(\theta)$ be an elementary submodel with $\mathscr{A} \in N$. We will say that $N$ is $\mathscr{A}$ covering if for every countable collection $\left\{b_{i}: i \in \omega\right\} \subset N \cap[\omega]^{\omega}$ of sets avoiding $\mathscr{A}$, there is $a \in N \cap \mathscr{A}$ such that $\forall i \in \omega\left[\left|a \cap b_{i}\right|=\omega\right]$.

Lemma 74. Let $\mathscr{A} \subset[\omega]^{\omega}$ be a strongly MAD family. There is $N \prec H(\theta)$ with $\mathscr{A} \in N$ such that:

(1) $|N|=\omega_{1}$ and $\omega_{1} \subset N$.

(2) $N$ is $\mathscr{A}$-covering.

Proof. We build $N$ as the union of an elementary chain of length $\omega_{1}$ of countable elementary submodels of $H(\theta)$. Thus we construct a chain $N_{0} \prec$ $N_{1} \prec \cdots \prec N_{\alpha} \prec \cdots \prec N_{\omega_{1}}=N \prec H(\theta)$, where $\left|N_{\alpha}\right|=\omega$, for all $\alpha<\omega_{1}$. To start with, we ensure $\mathscr{A} \in N_{0}$. Given $N_{\alpha}$, we ensure that $\alpha \in N_{\alpha+1}$ and that there is $a \in \mathscr{A} \cap N_{\alpha+1}$ such that $|a \cap b|=\omega$ for all $b \in[\omega]^{\omega} \cap N_{\alpha}$ avoiding $\mathscr{A}$. It is possible to do this because $\mathscr{A}$ is strongly MAD and because $\left|N_{\alpha}\right|=\omega$. Finally, if $\alpha$ is a limit, we set $N_{\alpha}=\bigcup_{\beta<\alpha} N_{\beta}$. Now, it is clear that $N=\bigcup_{\alpha<\omega_{1}} N_{\alpha}$ is as required.

THEOREM 75. ${ } \operatorname{FN}(\mathcal{P}(\omega))$ implies that all strongly MAD families in $[\omega]^{\omega}$ (and hence $\omega^{\omega}$ ) have size at most $\aleph_{1}$. In particular, in the Cohen model, all strongly MAD families in both $[\omega]^{\omega}$ and $\omega^{\omega}$ have size at most $\aleph_{1}$.

Proof. Let $\mathscr{A} \subset[\omega]^{\omega}$ be a strongly MAD family. Let $N \prec H(\theta)$ be $\mathscr{A}$ covering with $\omega_{1} \subset N$ and $|N|=\aleph_{1}$. We claim that $N \cap \mathscr{A}=\mathscr{A}$. Suppose for a contradiction that there is $a \in \mathscr{A}$ with $a \notin N$. Put $\bar{a}=\omega \backslash a$. Since $N \cap \mathcal{P}(\omega) \leq_{\sigma} \mathcal{P}(\omega)$, there is a countable set $C \subset N \cap \mathcal{P}(\bar{a})$ such that for all $b \in N \cap \mathcal{P}(\bar{a})$, there is $c \in C$ with $b \subset c \subset \bar{a}$. Notice that since $\mathscr{A}$ is an a.d. 
family, every $b \in N \cap \mathscr{A}$ is almost contained in $\bar{a}$. It follows that for every $b \in N \cap \mathscr{A}$, there is $c \in C$ such that $b \subset^{*} c$.

Now, we claim that for each $c \in C, \omega \backslash c$ avoids $\mathscr{A}$. Indeed, suppose not. Since $c \in N$, there is a finite set $\left\{a_{0}, \ldots, a_{k}\right\} \subset N \cap \mathscr{A}$ such that $\omega \backslash c \subset^{*} a_{0} \cup \cdots \cup a_{k}$. But $c \subset \bar{a}$, and so $a \subset \omega \backslash c \subset^{*} a_{0} \cup \cdots \cup a_{k}$. But this is impossible because $a \notin N$ and hence is a.d. from $a_{0}, \ldots, a_{k}$.

Thus we conclude that $\{\omega \backslash c: c \in C\}$ is a countable subset of $N \cap[\omega]^{\omega}$ of sets avoiding $\mathscr{A}$. Since $N$ is $\mathscr{A}$-covering, there is $b \in N \cap \mathscr{A}$ such that $|b \cap(\omega \backslash c)|=\omega$ for all $c \in C$. But we have argued above that $b \subset^{*} c$ for some $c \in C$. This a contradiction, which finishes the proof.

REMARK 76. Since strongly MAD families in $\omega^{\omega}$ are iterated Sacks and iterated Miller indestructible (Corollary 66), we can modify the elementary submodel argument alluded to at the beginning of this section to show that all strongly MAD families have size $\aleph_{1}$ in both the Sacks and Miller models.

8. Miscellaneous results. We will gather together here some assorted results that do not belong in any of the previous sections. Our first result grew out of a conversation with Michael Hrušák. For the case of MAD families in $[\omega]^{\omega}$, the notion of strong MADness (see Definition 70) turns out to be closely related to that of Cohen-indestructibility. It is shown in Hrušák and García Ferreira [9] and Kurilić [18] that a MAD family of subsets of $\omega$ is Cohen-indestructible iff it is "somewhere" strongly MAD. This led Hrušák to suggest that a similar result is true for MAD families of functions as well. We will show below that this is not the case. Indeed, we will show that assuming $\mathrm{CH}$, we can construct a Cohen-indestructible MAD family of functions that is "nowhere" van Douwen MAD (and hence "nowhere" strongly MAD). This shows that Cohen-indestructibility is somewhat different for MAD families of functions.

Definition 77. Let $\mathscr{A} \subset \omega^{\omega}$ be an a.d. family. Let $f \in \omega^{\omega}$. We define $\mathscr{A} \cap f=\{h \cap f: h \in \mathscr{A} \wedge|h \cap f|=\omega\}$. Note that this is an a.d. family on the countable set $f$. The trace of $\mathscr{A}$, written $\operatorname{tr}(\mathscr{A})$, is $\left\{f \in \omega^{\omega}: \mathscr{A} \cap f\right.$ is a MAD family in $\left.[f]^{\omega}\right\}$.

Definition 78. Let $\mathscr{A} \subset \omega^{\omega}$ be a MAD family. We say that $\mathscr{A}$ has trivial trace if no member of $\operatorname{tr}(\mathscr{A})$ avoids $\mathscr{A}$.

Theorem 79. Assume CH. There is a Cohen-indestructible MAD family $\mathscr{A} \subset \omega^{\omega}$ with trivial trace.

Proof. To ensure that our family is Cohen-indestructible, we will do a construction similar to the one in Kunen [17]. Let $\mathbb{P}=\operatorname{Fn}(\omega, 2)$. Since we are assuming $\mathrm{CH}$, there are only $\omega_{1}$ nice $\mathbb{P}$ names for elements of $\omega^{\omega}$. Let $\left\langle\left\langle p_{\alpha}, \dot{f}_{\alpha}\right\rangle: \alpha<\omega_{1}\right\rangle$ enumerate all pairs $\langle p, \dot{f}\rangle$ such that $p \in \mathbb{P}$ and $\dot{f}$ is a 
nice $\mathbb{P}$ name for an element of $\omega^{\omega}$. Let $\left\langle g_{\alpha}: \alpha<\omega_{1}\right\rangle$ enumerate $\omega^{\omega}$. An ideal $\mathcal{I}$ of subset of $\omega$ is said to be dense if $\forall a \in[\omega]^{\omega} \exists b \in[a]^{\omega}[b \in \mathcal{I}]$. Fix a proper, non-principal dense ideal on $\omega$. Notice that for any such ideal $\mathcal{I}$, if $\left\{a_{i}: i \in \omega\right\} \subset \mathcal{I}$ is a countable collection of infinite sets, then there is an infinite set $b \in \mathcal{I}$ such that $\forall i \in \omega\left[\left|b \cap a_{i}\right|<\omega\right]$. Now, we will build two sequences $\left\langle\mathscr{A}_{\alpha}: \alpha<\omega_{1}\right\rangle$ and $\left\langle\mathscr{B}_{\alpha}: \alpha<\omega_{1}\right\rangle$ such that the following hold:

(1) $\mathscr{A}_{\alpha} \subset \omega^{\omega}$ is a countable a.d. family.

(2) $\mathscr{B}_{\alpha}$ is a countable set of infinite partial functions.

(3) $\forall f \in \mathscr{B}_{\alpha}[\operatorname{dom}(f) \in \mathcal{I}]$.

(4) $\forall \alpha<\beta<\omega_{1}\left[\mathscr{A}_{\alpha} \subset \mathscr{A}_{\beta} \wedge \mathscr{B}_{\alpha} \subset \mathscr{B}_{\beta}\right]$.

(5) $\forall h \in \mathscr{A}_{\alpha} \forall f \in \mathscr{B}_{\alpha}[|h \cap f|<\omega]$.

(6) If $g_{\alpha}$ avoids $\bigcup\left\{\mathscr{A}_{\beta}: \beta<\alpha\right\}$, then there is $f \subset g_{\alpha}$ such that $f \in \mathscr{B}_{\alpha}$.

(7) If $p_{\alpha} \Vdash \stackrel{\circ}{f}_{\alpha}$ is a.d. from $\bigcup\left\{\mathscr{A}_{\beta}: \beta<\alpha\right\}$, then $\exists h \in \mathscr{A}_{\alpha}\left[p_{\alpha} \Vdash\left|h \cap \dot{\circ}_{\alpha}\right|\right.$ $=\omega]$.

Our MAD family $\mathscr{A}$ will be $\bigcup \mathscr{A}_{\alpha}$. It is clear from clauses (5) and (6) that $\mathscr{A}$ has trivial trace, while it is easy to see that clause (7) implies that $\mathscr{A}$ is Cohen-indestructible.

Assume that $\left\langle\mathscr{A}_{\beta}: \beta<\alpha\right\rangle$ and $\left\langle\mathscr{B}_{\beta}: \beta<\alpha\right\rangle$ have already been constructed. Set $\mathscr{C}=\bigcup \mathscr{A}_{\beta}$ and $\mathscr{B}=\bigcup \mathscr{B}_{\beta}$. Then $\mathscr{C} \subset \omega^{\omega}$ is a countable a.d. family and $\mathscr{B}$ is a countable family of infinite partial functions. Moreover, $\forall f \in \mathscr{B} \forall h \in \mathscr{C}[|h \cap f|<\omega]$. We will first define $\mathscr{B}_{\alpha}$, taking care of clause (6). Consider $g_{\alpha}$. If $g_{\alpha}$ does not avoid $\mathscr{C}$, there is nothing to be done, and we simply set $\mathscr{B}_{\alpha}=\mathscr{B}$. Now, let us assume that $g_{\alpha}$ avoids $\mathscr{C}$. Since $\mathscr{C}$ is countable, this assumption implies that $\mathscr{C} \cap g_{\alpha}$ is neither a finite nor an infinite MAD family on $g_{\alpha}$. So there is an infinite partial function $p \subset g_{\alpha}$ which is a.d. from $\mathscr{C}$. Since $\mathcal{I}$ is a dense ideal, there is an infinite partial function $f \subset p$ with $\operatorname{dom}(f) \in \mathcal{I}$. As $p$ is a.d. from $\mathscr{C}, f$ is also a.d. from $\mathscr{C}$, and therefore we can set $\mathscr{B}_{\alpha}=\mathscr{B} \cup\{f\}$.

Next, we define $\mathscr{A}_{\alpha}$. Once again, if $p_{\alpha} \nVdash \dot{f}_{\alpha}^{\circ}$ is a.d. from $\mathscr{C}$, there is nothing to be done, and we set $\mathscr{A}_{\alpha}=\mathscr{C}$. Now, assume that $p_{\alpha} \Vdash f_{\alpha}$ is a.d. from $\mathscr{C}$. Put $\mathscr{B}_{\alpha}=\left\{f_{i}: i \in \omega\right\}$ and $\mathscr{C}=\left\{h_{i}: i \in \omega\right\}$. For each $i \in \omega$, put $a_{i}=\operatorname{dom}\left(f_{i}\right)$. Thus $\left\{a_{i}: i \in \omega\right\}$ is a countable collection of infinite sets in $\mathcal{I}$. By our observation above, there is an infinite set $b \in \mathcal{I}$ such that $\forall i \in \omega\left[\left|b \cap a_{i}\right|<\omega\right]$. We will define an infinite partial function $h^{0}$ with $\operatorname{dom}\left(h^{0}\right) \subset b$ such that $p_{\alpha} \Vdash\left|h^{0} \cap \dot{f}_{\alpha}\right|=\omega$. Observe that for any $i \in \omega, h^{0} \cap f_{i}$ will be finite. To get $h^{0}$ we proceed as follows. Let $\left\{q_{i}: i \in \omega\right\}$ enumerate $\left\{q \in \mathbb{P}: q \leq p_{\alpha}\right\}$. We will build $h^{0}$ as the union of an increasing sequence of finite partial functions. We will build a sequence $\left\langle h_{i}^{0}:-1 \leq i<\omega\right\rangle$ such that: 
(a) $h_{-1}^{0}=0$ and $h_{i}^{0}$ is a finite partial function with $\operatorname{dom}\left(h_{i}^{0}\right) \subset b$.

(b) $h_{i-1}^{0} \subset h_{i}^{0}$ and $\forall j \leq i\left[h_{i}^{0} \cap h_{j} \subset h_{i-1}^{0} \cap h_{j}\right]$.

(c) $\exists k_{i} \geq i \exists r \leq q_{i}\left[k_{i} \in \operatorname{dom}\left(h_{i}^{0}\right) \wedge r \Vdash h_{i}^{0}\left(k_{i}\right)=\stackrel{\circ}{f}_{\alpha}\left(k_{i}\right)\right]$.

Put $h^{0}=\bigcup h_{i}^{0}$. It is clear from clause (b) that $h^{0}$ is a.d. from $h_{i}$ for all $i \in \omega$. Also, we see from clause (a) that $\operatorname{dom}\left(h^{0}\right) \subset b$. We will argue that $p_{\alpha} \Vdash\left|h^{0} \cap \dot{f}_{\alpha}\right|=\omega$. Let $q \leq p_{\alpha}$ and let $n \in \omega$. There are infinitely many conditions below $q$. Hence we can find $i>n$ such that $q_{i} \leq q$. But now by clause (c) there are $k_{i} \geq i>n$ and $r \leq q_{i} \leq q$ such that $k_{i} \in \operatorname{dom}\left(h_{i}^{0}\right) \subset$ $\operatorname{dom}\left(h^{0}\right)$ and $r \Vdash \stackrel{\circ}{f}_{\alpha}\left(k_{i}\right)=h_{i}^{0}\left(k_{i}\right)=h^{0}\left(k_{i}\right)$.

We will now describe how to construct $\left\langle h_{i}^{0}:-1 \leq i<\omega\right\rangle . h_{-1}^{0}$ is 0 . At stage $i \geq 0$, assume that $h_{i-1}^{0}$ is given to us. We wish to define $h_{i}^{0}$ so that clause (c) is satisfied. But we need to be sure that we introduce no new agreements between $h_{i}^{0}$ and any of the members of $\left\{h_{0}, \ldots, h_{i}\right\}$. We know that $q_{i} \Vdash f_{\alpha}^{\circ}$ is a.d. from $\left\{h_{0}, \ldots, h_{i}\right\}$. Hence, there is $\tilde{r} \leq q_{i}$ and $l \in \omega$ such that $\tilde{r} \Vdash \forall k>l\left[\dot{f}_{\alpha}(k) \notin\left\{h_{0}(k), \ldots, h_{i}(k)\right\}\right]$. Put $m=\max \left(\operatorname{dom}\left(h_{i-1}^{0}\right)\right)$. Since $b$ is an infinite set there is a $k_{i} \in b$ with $k_{i}>\max \{m, l, i\}$. Now, since $\AA_{\alpha}$ is a name for an element of $\omega^{\omega}$, we can find $r \leq \tilde{r}$ and $n \in \omega$ such that $r \Vdash$ $\stackrel{\circ}{f}_{\alpha}\left(k_{i}\right)=n$. Notice that our choice of $\tilde{r}$ entails that $n \notin\left\{h_{0}\left(k_{i}\right), \ldots, h_{i}\left(k_{i}\right)\right\}$. Since $k_{i}>m$, we can define $h_{i}^{0}=h_{i-1}^{0} \cup\left\{\left\langle k_{i}, n\right\rangle\right\}$. As $k_{i} \in b$, this is as required.

We are almost done. We just need to extend $h^{0}$ to a total function. Since both $\mathscr{B}_{\alpha}$ and $\mathscr{C}$ are countable, there is a total function $h^{\prime} \in \omega^{\omega}$ such that $\forall i \in \omega\left[\left|h^{\prime} \cap f_{i}\right|<\omega \wedge\left|h^{\prime} \cap h_{i}\right|<\omega\right]$. Put $X=\operatorname{dom}\left(h^{0}\right)$ and $Y=\omega \backslash X$. Put $h^{1}=h^{\prime} \uparrow Y$ and set $h=h^{0} \cup h^{1}$. It is clear that $h$ is a.d. from both $\mathscr{B}_{\alpha}$ and $\mathscr{C}$. So we may set $\mathscr{A}_{\alpha}=\mathscr{C} \cup\{h\}$, and this ends the proof.

Despite certain differences, there are close connections between the notion of a strongly MAD family of functions and the notion of a strongly MAD family of sets. Lemma 71 shows that the existence of the former implies the existence of the latter. We also get a connection between the indestructibility properties of strongly MAD families in $\omega^{\omega}$ and those of strongly MAD families in $[\omega]^{\omega}$.

Lemma 80. Let $\mathbb{P}$ be any poset. Suppose that any strongly MAD family in $[\omega]^{\omega}$ is strongly $\mathbb{P}$-indestructible (see Definition 26). Let $\mathscr{A} \subset \omega^{\omega}$ be strongly $M A D$. Then $\mathscr{A}$ is strongly $\mathbb{P}$-indestructible.

Proof. As in Lemma 71, let $C_{n}$ be the $n$th vertical column of $\omega \times \omega$. We know from Lemma 71 that $\mathscr{A} \cup\left\{C_{n}: n \in \omega\right\}$ is a strongly MAD family in $[\omega \times \omega]^{\omega}$. Now, let $G$ be a $(\mathbf{V}, \mathbb{P})$ generic filter. By assumption, in $\mathbf{V}[G]$, $\mathscr{A} \cup\left\{C_{n}: n \in \omega\right\}$ remains a strongly MAD family in $[\omega \times \omega]^{\omega}$. In $\mathbf{V}[G]$, let $\left\{f_{i}: i \in \omega\right\} \subset \omega^{\omega}$ be a countable family avoiding $\mathscr{A}$. As each $f_{i}$ is a.d. from 
each $C_{n}$, it follows that $\left\{f_{i}: i \in \omega\right\} \subset[\omega \times \omega]^{\omega}$ still avoids $\mathscr{A} \cup\left\{C_{n}: n \in \omega\right\}$. But then there must be $h \in \mathscr{A}$ such that $\forall i \in \omega\left[\left|h \cap f_{i}\right|=\omega\right]$.

Acknowledgements. The author thanks the anonymous referee for carefully reading the paper and making very useful suggestions.

The author was partially supported by NSF Grant DMS-0456653.

\section{References}

[1] U. Abraham, Proper forcing, in: Handbook of Set Theory, to appear.

[2] T. Bartoszyński, Combinatorial aspects of measure and category, Fund. Math. 127 (1987), 225-239.

[3] T. Bartoszyński and H. Judah, Set Theory: On the Structure of the Real Line, A K Peters, Wellesley, MA, 1995.

[4] J. Brendle and S. Yatabe, Forcing indestructibility of MAD families, Ann. Pure Appl. Logic 132 (2005), 271-312.

[5] A. Dow, An introduction to applications of elementary submodels to topology, Topology Proc. 13 (1988), 17-72.

[6] S. Fuchino, S. Koppelberg, and S. Shelah, Partial orderings with the weak FreeseNation property, Ann. Pure Appl. Logic 80 (1996), 35-54.

[7] M. Goldstern, Tools for your forcing construction, in: Set Theory of the Reals (Ramat Gan, 1991), Israel Math. Conf. Proc., Bar-Ilan Univ., Ramat Gan, 1993, 305360 .

[8] M. Hrušák, MAD families and the rationals, Comment. Math. Univ. Carolin. 42 (2001), 345-352.

[9] M. Hrušák and S. García Ferreira, Ordering MAD families a la Katětov, J. Symbolic Logic 68 (2003), 1337-1353.

[10] I. Juhász, L. Soukup, and Z. Szentmiklóssy, Combinatorial principles from adding Cohen reals, in: Logic Colloquium '95 (Haifa), Lecture Notes in Logic 11, Springer, Berlin, 1998, 79-103.

[11] - What is left of $\mathrm{CH}$ after you add Cohen reals?, in: 8th Prague Topological Symposium on General Topology and Its Relations to Modern Analysis and Algebra (1996), Topology Appl. 85 (1998), 165-174 .

[12] I. Juhász and K. Kunen, The power set of $\omega$. Elementary submodels and weakenings of $\mathrm{CH}$, Fund. Math. 170 (2001), 257-265.

[13] A. Kamburelis and B. Węglorz, Splittings, Arch. Math. Logic 35 (1996), 263-277.

[14] B. Kastermans, Very mad families, in: Advances in Logic, Contemp. Math. 425, Amer. Math. Soc., Providence, RI, 2007, 105-112.

[15] B. Kastermans, J. Steprāns, and Y. Zhang, Analytic and coanalytic families of almost disjoint functions, J. Symbolic Logic 73 (2008), 1158-1172.

[16] J. Kellner and S. Shelah, Preserving preservation, ibid. 70 (2005), 914-945.

[17] K. Kunen, Set Theory: An Introduction to Independence Proofs, Stud. Logic Found. Math. 102, North-Holland, Amsterdam, 1980.

[18] M. S. Kurilić, Cohen-stable families of subsets of integers, J. Symbolic Logic 66 (2001), 257-270.

[19] V. I. Malykhin, Topological properties of Cohen generic extensions, Trudy Moskov. Mat. Obshch. 52 (1989), 3-33, 247 (in Russian).

[20] A. W. Miller, Arnie Miller's problem list, in: Set Theory of the Reals (Ramat Gan, 1991), Israel Math. Conf. Proc. 6, Bar-Ilan Univ., Ramat Gan, 1993, 645-654. 
[21] D. Raghavan, There is a van Douwen mad family, Trans. Amer. Math. Soc., to appear.

[22] M. Repický, Goldstern-Judah-Shelah preservation theorem for countable support iterations, Fund. Math. 144 (1994), 55-72.

[23] S. Shelah, Proper and Improper Forcing, 2nd ed., Perspectives in Math. Logic, Springer, Berlin, 1998.

Department of Mathematics

University of Toronto

Toronto, ON M5S 2E4 Canada

E-mail: raghavan@math.toronto.edu

http://www.math.toronto.edu/raghavan

Received 8 February 2009;

in revised form 1 May 2009 